\title{
Rating autonomous systems
}

\section{Master Thesis}

\section{Author(s):}

Zimmerli, Laurent

Publication date:

2008

Permanent link:

https://doi.org/10.3929/ethz-a-005729053

Rights / license:

In Copyright - Non-Commercial Use Permitted 


\title{
EIH
}

Eidgenössische Technische Hochschule Zürich

Swiss Federal Institute of Technology Zurich

\section{Rating Autonomous Systems}

\author{
Laurent Zimmerli
}

Master's Thesis (MA-2008-07)

March 2008 - August 2008

Department of Computer Engineering and Networks Laboratory (TIK), ETH Zurich

Conducted at Open Systems AG

Tutor: $\quad$ Christoph Göldi (Open Systems AG)

ETH-Tutor: Bernhard Tellenbach

Supervisor: Prof. Dr. Bernhard Plattner 


\begin{abstract}
Many business and research applications operate geographically distributed on various sites connected over the Internet. Such applications rely on the availability and performance of the Internet connections between the sites. Bad quality of the Internet directly impacts their performance.
\end{abstract}

The Internet is a network built from several subnetworks connected to each other. Those subnetworks are called Autonomous Systems. The quality of an end-to-end connection over the Internet depends on the quality of the networks the connection traverses, i.e., it depends on the traversed Autonomous Systems.

Autonomous Systems cannot guarantee any performance quality. Experiencing connection problems an Internet user must debug manually to spot the problem source. Furthermore it has turned out to be difficult to chose between local ISPs-which typically maintain an own Autonomous System — due to the lack of service level agreements.

In this thesis, we develop an approach to rate Autonomous Systems by their quality. We define a way to detect the Autonomous System which causes the bad performance on a slow Internet connection in real-time. Furthermore we rate the quality of their network performance as well as the quality of their peerings during five weeks.

The rating is based on traceroute measurements among numerous measurement points. Every Autonmous System which is traversed by the measurements is rated. To test our approach we performed 16 millions measurements from 31 sources to 159 destinations during 38 days.

Our rating approach results in ordered rankings of the network as well as the peering quality of the Autonomous Systems. Furthermore scatter plots allow graphical comparison of the qualities.

Unfortunately we can neither be sure about the reliability of our rating nor about the correct detection of Autonomous Systems causing bad Internet performance. This is because on the one hand we experienced general problems with traceroute measurements and on the other hand we have no possibility to verify our rating results. 


\section{Kurzfassung}

Viele Geschäfts- und Forschungsapplikationen werden auf geographisch verteilten Computern betrieben, die über das Internet miteinander verbunden sind. Solche Anwendungen sind auf die Verfügbarkeit und Effizienz der Internetverbindungen zwischen den Rechnern angewiesen, d.h. schlechte Qualität des Internets beeinflusst die Leistung solcher Applikationen unmittelbar.

Das Internet ist ein Netzwerk, welches aus vielen verschiedenen Teilnetzwerken-sogenannten Autonomen Systemen — besteht, die miteinander verbunden sind. Die Qualität einer End-zu-End Verbindung über das Internet hängt von der Qualität der Teilnetzwerke ab, welche von der Verbindung durchlaufen werden; die Qualität basiert also auf der Qualität der traversierten Autonomen Systeme.

Autonome Systeme können keine Qualität ihrer Leistung garantieren. Treten Verbindungsprobleme auf, muss ein Internet-Benutzer diesen selbst nachgehen, um die Ursache zu finden. Wegen der Ungewissheit über diese Qualitäten hat es sich ausserdem als schwierig erwiesen, zwischen lokalen ISPs—welche normalerweise ein eigenes Autonomes System betreiben—auszuwählen.

In dieser Masterarbeit entwickeln wir einen Ansatz, um Autonome Systeme anhand ihrer Qualität zu bewerten. Wir definieren eine Methode, um in Echtzeit das Autonome System zu ermittlen, welches die schlechte Leistung einer Internetverbindung verursacht. Ausserdem bewerten wir die Güte der Netzwerkleistung und die Qualität der Peerings von Autonomen Systemen über eine Zeitspanne von fünf Wochen.

Die Bewertung basiert auf traceroute Messungen zwischen einer Vielzahl von Messpunkten. Jedes Autonome System, welches von einer Messung traversiert wird, fällt in die Bewertung. Um unseren Ansatz zu testen, führten wir während 38 Tagen von 31 Start- zu 159 Zielpunkten insgesamt 16 Millionen Messungen aus.

Unser Bewertungansatz produziert Ranglisten der Netzwerk- und Peeringqualität der Autonomen Systeme und stellt Scatterplots her, auf welchen man graphisch die Qualitäten vergleichen kann.

Leider können wir über die Verlässlichkeit unserer Bewertung und unseres Ansatzes, Autonome Systeme zu bestimmen, welche schlechte End-zu-End Verbindungen verursachen, keine Aussage machen. Der Grund dafür sind einerseits Probleme, die wir mit den traceroute Messungen erfuhren, und andererseits die Unmöglichkeit, unsere Bewertungsresultate zu verifizieren. 


\section{Contents}

$\begin{array}{lll}1 & \text { Introduction } & 1\end{array}$

1.1 Motivation . . . . . . . . . . . . . . . . . . . . . . . . 1

1.2 Task . . . . . . . . . . . . . . . . . . . . 2

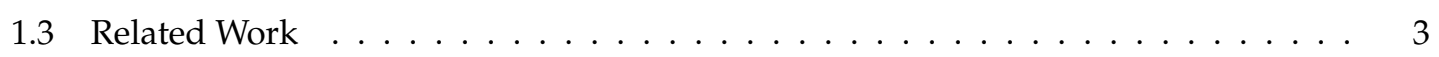

1.3 .1 Autonomous Systems $\ldots \ldots \ldots \ldots \ldots$

1.3 .2 Network Performance . . . . . . . . . . . . . . . . . . . . . . 3

1.3 .3 Network Measurements . . . . . . . . . . . . . . . 3

$1.3 .4 \quad$ Data Mining for Network Analysis . . . . . . . . . . . . . . . . . 3

1.3 .5 Related Work at Open Systems . . . . . . . . . . . . . . . . . 4

1.4 Overview . . . . . . . . . . . . . . . . . . . . 4

\begin{tabular}{|lll}
2 & Terms and Definitions & 5
\end{tabular}

$2.1 \quad$ Autonomous System . . . . . . . . . . . . . . . . . . . . . 5

2.2 Internet User . . . . . . . . . . . . . . . . . . . 6

2.3 Network Performance $\ldots \ldots \ldots \ldots \ldots$. . . . . . . . . . . . . . 6

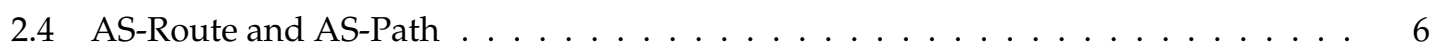

\begin{tabular}{|ll|l}
3 & AS Quality Indicators & 7
\end{tabular}

3.1 AS Quality . . . . . . . . . . . . . . . . . . . . . . . 7

3.2 Intra-AS vs. Inter-AS Quality $\ldots \ldots \ldots \ldots \ldots \ldots \ldots \ldots$

3.3 Intra-AS Quality: Network Performance. . . . . . . . . . . . . . . . . . 8

$3.3 .1 \quad$ IP Performance $\ldots \ldots \ldots \ldots \ldots \ldots$

3.3 .2 Internal Path $\ldots \ldots \ldots \ldots \ldots \ldots$

3.4 Inter-AS Quality: Peering $\ldots \ldots \ldots \ldots \ldots \ldots \ldots$

$3.4 .1 \quad$ Uplink and Peer Qualities . . . . . . . . . . . . . . . . . . . . 11

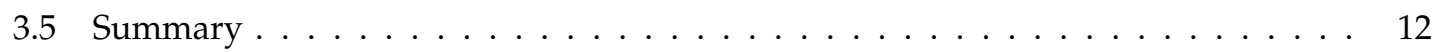


\begin{tabular}{lll}
\hline & AS Quality Rating & 13
\end{tabular}

4.1 Measurements . . . . . . . . . . . . . . . . . . . . . . . . . . . . . . 13

$4.1 .1 \quad$ AS Measurements . . . . . . . . . . . . . . . . . . . . . . . . . . . . . . 14

4.2 Rating. . . . . . . . . . . . . . . . . . . . . . . 14

$4.2 .1 \quad$ Data Mining . . . . . . . . . . . . . . . . . . . . . . . . . . . . . 15

4.2 .2 Comparing Quality Measures . . . . . . . . . . . . . . . . 16

4.3 Statistical Quality Measures $\ldots \ldots \ldots$. . . . . . . . . . . . . . . . 16

$4.3 .1 \quad$ Delay Stability $\ldots \ldots \ldots \ldots \ldots$

4.3 .2 Delay per Length $\ldots \ldots \ldots \ldots \ldots \ldots$

4.3 .3 Internal Loss. . . . . . . . . . . . . . . . . . . . . . . . . . 18

$4.3 .4 \quad$ Border $\operatorname{Loss} \ldots \ldots \ldots \ldots \ldots \ldots \ldots$

4.3 .5 Internal Length Stability $\ldots \ldots \ldots \ldots \ldots$

4.3 .6 Outgoing AS-Path $\ldots \ldots \ldots \ldots \ldots \ldots$

4.4 GeolP Accuarcy . . . . . . . . . . . . . . . . . . . . . . . . . . . . . . . . . . . . . 19

4.5 Measurement Problem . . . . . . . . . . . . . . . . . . . . . . . . . . . . . 19

4.5 .1 Observations . . . . . . . . . . . . . . . . . . . . . . . . . . . . . . 19

4.5 .2 Possible Causes . . . . . . . . . . . . . . . . . . . . . . . 20

4.5 .3 Consequences . . . . . . . . . . . . . . . . . . . . . . . 20

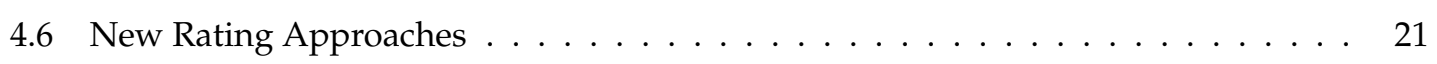

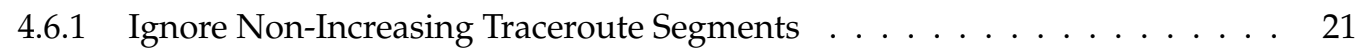

4.6 .2 Outlier-based Measurement Interpretation . . . . . . . . . . . . . . . 21

5 Specification of the Framework 27

5.1 Data Collection . . . . . . . . . . . . . . . . . . . 27

$5.1 .1 \quad$ Measurement Points . . . . . . . . . . . . . . . . . . . . . . . . . . 27

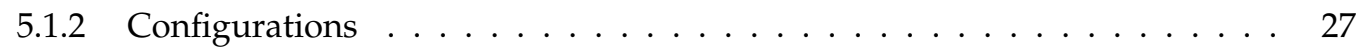

5.1 .3 Network Measurement Tools . . . . . . . . . . . . . . . . . . . . . . 28

5.2 Storage . . . . . . . . . . . . . . . . . . . . . . . . . . . . . . . 29

$5.2 .1 \quad$ Initial Approach $\ldots \ldots \ldots \ldots$

$5.2 .2 \quad$ New Approach $\ldots \ldots \ldots \ldots$. . . . . . . . . . . . . . . . . 30

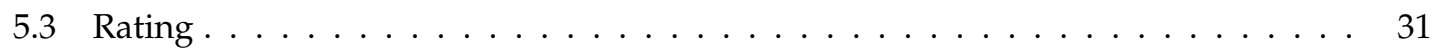

6 Implementation 33

6.1 Data Collection . . . . . . . . . . . . . . . . . . . . . . . 33

$6.1 .1 \quad$ Measurement Intervals . . . . . . . . . . . . . . . . . . . . . . . . . . 33 
6.2 Database . . . . . . . . . . . . . . . . . . . . 34

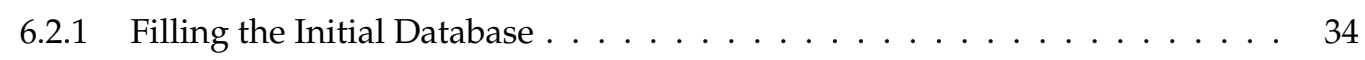

6.2 .2 Filling the New Database $\ldots \ldots \ldots \ldots \ldots$. . . . . . . . . 34

6.3 Data Evaluation . . . . . . . . . . . . . . . . . . . . . . . . . . 35

6.3 .1 Initial Tests . . . . . . . . . . . . . . . . . . . . . 35

6.3 .2 Rating . . . . . . . . . . . . . . . . . 35

\begin{tabular}{lll}
\hline 7 & Evaluation & 37
\end{tabular}

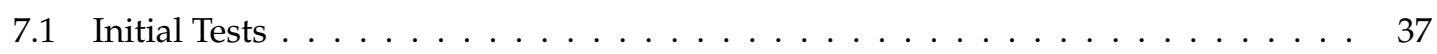

$7.1 .1 \quad$ RTT Distribution . . . . . . . . . . . . . . . . . . . 37

7.1 .2 Interquartile Range . . . . . . . . . . . . . . . . . . . . . . . 39

$7.1 .3 \quad$ AS-RTT . . . . . . . . . . . . . . . . . . . . . . . . 39

7.1 .4 Traceroute Configurations . . . . . . . . . . . . . . . . 40

7.2 Evaluating the Rating Approach $\ldots \ldots \ldots \ldots \ldots \ldots \ldots \ldots$

$7.2 .1 \quad$ Overall Statistics . . . . . . . . . . . . . . . . . . . . . . . . 40

7.2 .2 Intra-AS Rating . . . . . . . . . . . . . . . . . . 41

7.2 .3 Inter-AS Rating . . . . . . . . . . . . . . . . . . . . 42

$7.2 .4 \quad$ AS Rating for ISP Selection $\ldots \ldots \ldots \ldots$

$7.2 .5 \quad$ AS Rating for Real-Time Debugging $\ldots \ldots \ldots \ldots$

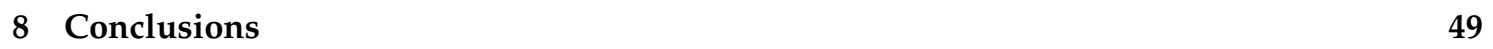

8.1 Summary . . . . . . . . . . . . . . . . . . . . . . . . . . . . . 49

8.2 Conclusions . . . . . . . . . . . . . . . . . . . . . . . . . . 49

$8.3 \quad$ Future Work . . . . . . . . . . . . . . . . . . . . . . . . . . . . 50

8.3 .1 Traceroute . . . . . . . . . . . . . . . . . . . 50

8.3 .2 Enhancing our Rating Approach $\ldots \ldots \ldots \ldots$. . . . . . . . . 50

8.3 .3 Alternative Rating Approach . . . . . . . . . . . . . . . . . . 50

$8.3 .4 \quad$ Implementation for use in Practice . . . . . . . . . . . . . . . . 50

8.3 .5 Long-Term Evaluation . . . . . . . . . . . . . . . . . . . 50

8.4 Application in Practice $\ldots \ldots \ldots \ldots \ldots$. . . . . . . . . . . . . . . . 51

A Database Fields 53

A.1 Initial Database Scheme . . . . . . . . . . . . . . . . . . . . 53

A.1.1 Table "autonomous_system" . . . . . . . . . . . . . . . . . . 53

A.1.2 Table"config" . . . . . . . . . . . . . . . . . . . . . . . 54 
A.1.3 Table "end_host" . . . . . . . . . . . . . . . . . . . . . . . 54

A.1.4 Table "host" . . . . . . . . . . . . . . . . . . . . . . . . . 54

A.1.5 Table "measurement" . . . . . . . . . . . . . . . . . . . . . . 54

A.2 New Database Scheme . . . . . . . . . . . . . . . . . . . . . . . 55

A.2.1 Table"as_route" . . . . . . . . . . . . . . . . . . . 55

A.2.2 Table" $\mathrm{e} 2 \mathrm{e} "$. . . . . . . . . . . . . . . . . . . . . . 55

A.2.3 Table "measurement" . . . . . . . . . . . . . . . . . . 56

A.3 Extension of the new Database $\ldots \ldots \ldots \ldots \ldots \ldots$. . . . . . . . . 56

A.3.1 Table"outlier_as" . . . . . . . . . . . . . . . . . . . . . 56

A.3.2 Table"outlier_unknown" . . . . . . . . . . . . . . . . . . . 56

A.3.3 Table "unreached" . . . . . . . . . . . . . . . . . . . . . 57

\begin{tabular}{ll}
\hline B Software Versions & 59
\end{tabular}

\begin{tabular}{ll}
\hline C Data Evaluation Queries & 61
\end{tabular}

C.1 Overall Statistics. . . . . . . . . . . . . . . . . . . . . . 61

C.2 Intra-AS Quality . . . . . . . . . . . . . . . . . . . . 62

C.3 Inter-AS Quality . . . . . . . . . . . . . . . . . . . . . . 63

C.4 AS End-to-End Rating . . . . . . . . . . . . . . . . . . . . . . . . 64

\begin{tabular}{ll}
\hline D AS Rankings & 65
\end{tabular}

D.1 Intra-AS Quality . . . . . . . . . . . . . . . . . . . . . . . . . 65

D.2 Inter-AS Quality . . . . . . . . . . . . . . . . . . . 71

\begin{tabular}{lll}
\hline E & Scatter Plots & 75
\end{tabular}

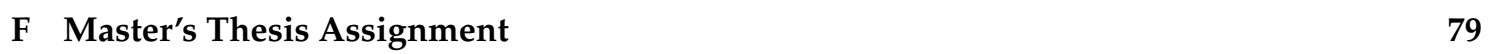

\begin{tabular}{lr}
\hline G Schedule & 85
\end{tabular}

\begin{tabular}{lr}
\hline References & 85
\end{tabular} 


\title{
Abbreviations
}

\author{
AS Autonomous system \\ ASN Autonomous system number \\ BGP Border Gateway Protocol \\ CAIDA Cooperative Association for Internet Data Analysis \\ CCDF Complementary Cumulative Distribution Function \\ CDF Cumulative Distribution Function \\ COV Coefficient of Variation \\ CPU Central Processing Unit \\ DDM Distributed Data Mining \\ ER Entity-Relationship \\ HTTP Hypertext Transfer Protocol \\ ICMP Internet Control Message Protocol \\ IETF Internet Engineering Task Force \\ IP Internet Protocol \\ IQR Interquartile Range \\ ISP Internet Service Provider \\ MTU Maximum Transmission Unit \\ OSI Open Systems Interconnection \\ PASTA Poisson Arrivals See Time Averages \\ RFC Request For Comments \\ RTT Round-Trip Time \\ SNMP Simple Network Management Protocol \\ TCP Transmission Control Protocol
}


TTL Time-to-Live

UCLA University of California, Los Angeles

VPN Virtual Private Network 


\section{Chapter 1}

\section{Introduction}

\subsection{Motivation}

Many business and research applications operate geographically distributed on various sites connected over the Internet. Such applications rely on the availability and performance of the Internet connections between the sites. Bad quality of the Internet directly impacts their performance.

The Internet is basically a network of networks, it consists of many subnetworks which are connected among each other. These subnetworks are called Autonomous Systems (AS). Each Internet connection passes through one or several Autonomous Systems as illustrated in Fig. 1.1 The blue circles represent Autonomous Systems which are traversed by the connection between the two end-hosts. The quality of an Internet connection relies on the network quality of the traversed Autonomous Systems.

As a Managed Security Service Provider, Open Systems operates large Virtual Private Networks (VPNs) spanned across the world. VPNs are built on top of the Internet and therefore strongly rely on the performance of the underlying network connections. Manual analysis of quality

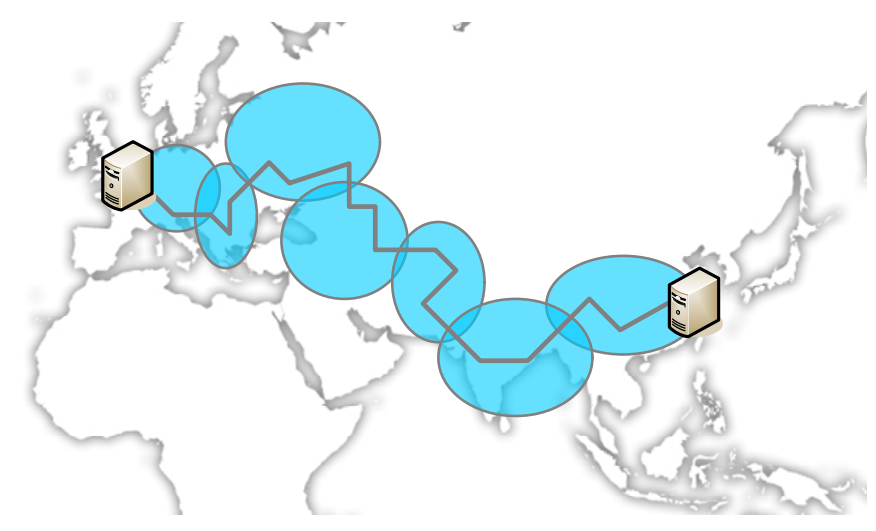

Figure 1.1: End-to-end connection traversing multiple Autonomous Systems (blue circles) 


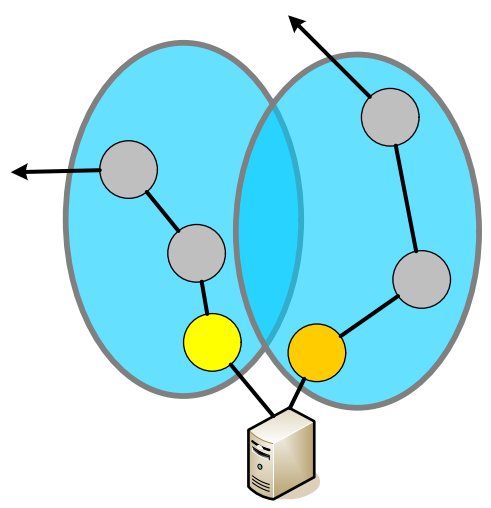

Figure 1.2: VPN location with access to different ISPs in different AS's

issues with VPN connections is time consuming and does not scale well with large VPN environments consisting of hundreds of sites located around the world. Open Systems has developed several tools to measure network connection quality and ease network debugging when problems appear. However, debugging still remains a time consuming task and extensive manual analysis is needed to get a long-term benchmark for individual Autonomous Systems.

\subsection{Task}

Given the above motivation, this master's thesis approaches the following research question:

How can Autonomous Systems automatically, efficiently, and in a meaningful and reliable way be rated by their quality in real-time as well as over long periods of time?

The goal of the thesis is a framework which consists of two parts:

1. A system which rates the quality of an AS on a specific end-to-end route in the Internet on demand and in real-time

2. A long-term rating of the overall quality of an AS in a well-defined context

The first part enables Internet users to spot current weak points of Internet connections and find reasons for poor network performance. The second part enables Internet users to evaluate the quality of an Autonomous System within their context over extended periods of time.

Real-time rating supports Open Systems debugging ongoing connectivity problems. Once the origin of a poor Internet connection is known, the operator of this particular AS can be urged to solve the problem. Long-term AS rating helps to determine generally bad performing networks. Once known, Open Systems can try to avoid weak AS's in their VPNs, e.g., by selecting the best ISP for a given VPN location (cf. Fig. 1.2). 


\subsection{Related Work}

There has been several research projects in the direction of our task, but no paper has been found concretely addressing our research question. However, there is related work whose findings were reused for this thesis.

\subsubsection{Autonomous Systems}

Alves et al. [1] characterized the Internet connections evolution over the last 10 years at the Autonomous Systems level analyzing BGP data. Dimitropoulos et al. [2] introduced heuristics for inferring AS relationships-which delivered better results than from BGP data-and offers a data repository at CAIDA $\sqrt{1}$ containing up-to-date data about AS relationships. Dimitropoulos et al. [3] also classified AS's and calculated relevant characteristics for all current AS's from that time. Wang et al. ranked Autonomous Systems according to their routing contribution to the Internet using their IDAV model [4].

All of these papers relate to characteristics of AS's, but do not study the quality of Autonomous Systems. No related work has been found which actually rates Autonomous Systems according to their quality or even defines quality measures.

\subsubsection{Network Performance}

Chen et al. [5] surveyed different projects and tools for Internet performance monitoring. Prasad et al. [6] surveyed metrics, measurement techniques, and tools for bandwidth estimation.

Harfoush et al. [7] developed an improved method to measure hop-by-hop bandwidth.

These papers are closely related to this thesis. Chapter 3 discusses how their findings are reused for our work.

\subsubsection{Network Measurements}

Augustin et al. [8] describe a number of anomalies that arise in nearly all traceroute-based measurements and provide a new publicly-available traceroute, called Paris traceroute, which controls packet header contents to obtain a more precise picture of the actual routes that packets follow. Augustin's tool is one of the tools which is used to collect data for this thesis as described in Sect. 5.1.3

Baccelli et al. [9] show that due to the diversity of aims and analysis techniques used in active probing, the benefits of Poisson based measurement, and the utility and role of PASTA, are unclear. Besides best practices from the IETF (cf. RFC 2330 [10]) Baccelli's findings are used to argue the usage of uniformly distributed measurement intervals as described in Sect. 6.1.1.

\subsubsection{Data Mining for Network Analysis}

Borzemski et al. [11] used data mining to predict the performance of data transfers. Borzemski [12] also developed a data mining decision model which can predict RTT and throughput

\footnotetext{
${ }^{1}$ Cooperative Association for Internet Data Analysis (CAIDA). http://www. caida.org/. last visit August 2008
} 
behavior when downloading Web objects. Baldi et al. [13] used data mining techniques for traffic analysis by extracting information from network packet records.

Borzemski used data mining for performance predictions. Our approach is not to predict, but to rate the current and past performance. Baldi's approach is more closely related to our task.

However, their approach requires significant amounts of captured data. Applying it to our task, large amounts of data would have to be captured on each of our measurement points. This does not scale well to a large number of measurement points.

\subsubsection{Related Work at Open Systems}

Janneth Malibago [14] monitored and analyzed Internet route changes trying to correlate them with network performance changes. In some cases correlations could be observed. Her findings are used for our work as described in section 3.4

\subsection{Overview}

The thesis is organized as follows: Chapter2 defines and explains important terms used throughout the thesis. Chapter 3 discusses candidate indicators for assessing the quality of Autonomous Systems whereas Chap. 4 explains how the quality indicators are used to actually rate an Autonomous System. Chapter 5 specifies the framework developed to perform the measurements and the rating, while Chap. 6 describes its implementation. Chapter 7 discusses the evaluation of the measurement data and the application of our rating approach, and Chap. 8 concludes the thesis and suggests future work. 


\section{Chapter 2}

\section{Terms and Definitions}

This chapter defines and explains important terms used throughout the thesis.

\subsection{Autonomous System}

An Autonomous System is a collection of IP networks with common routing policies. Every AS is identified by a unique Autonomous System number (ASN). Dividing the Internet in Autonomous Systems makes routing more scalable due to the decreasing need of both memory for and exchange of routing information. Transmitting network topology on a network-level instead of a router-level reduces the amount of data dramatically [15].

There are two different precise definitions of Autonomous Systems in RFC documents. In RFC 1930 [16] an Autonomous System (AS) is defined as follows:

An AS is a connected group of one or more IP prefixes run by one or more network operators which has a single and clearly defined routing policy.

RFC 1812 [17] defines it as follows:

An Autonomous System (AS) is a connected segment of a network topology that consists of a collection of subnetworks (with hosts attached) interconnected by a set of routes.

For the context of this thesis, however, a more high-level definition is sufficient:

Autonomous Systems are subnetworks which are connected among each other and together build the Internet.

There are different kinds of connections between Autonomous Systems:

- Customer-provider connections where one AS pays the other for data exchange and connection to other AS's 
- Peer connections where two AS's exchange data based on a peering-agreement (usually without any paying involved)

Connections to other AS's enable an AS to reach the "rest of the Internet". Routing between Autonomous Systems (inter-AS-routing) is standardized and must be done using an exterior routing protocol. Currently the only exterior routing protocol in use is the border gateway protocol (BGP). Routing within an AS (intra-AS-routing), however, is not standardized an the issue of the provider of the AS [15].

\subsection{Internet User}

There is no agreed definition of the term "Internet user". We use the term Internet user to denote any person, organization, or business which uses any kind of service that relies on the Internet.

\subsection{Network Performance}

According to the Teletraffic Engineering Handbook [18], network performance is defined as follows:

Network performance concerns the ability of a network or network portion to provide the functions related to communications between users. [...]

Section 3.3.1 elaborates which metrics we use to measure network performance.

\subsection{AS-Route and AS-Path}

$\mathrm{n}$ end-to-end Internet connection traverses one or more Autonomous Systems. The AS numbers of the traversed AS's ordered by their occurrence on an end-to-end connection are referred to as the AS-route or the AS-path. The terms AS-route and AS-path are used synonymic throughout this thesis. E.g., if a measurement traverses the AS's 9831, 15412, 174, and 16206 in the given order, then the corresponding AS-route is "9831-15412-174-16206". 


\section{Chapter 3}

\section{AS Quality Indicators}

This chapter discusses candidate indicators for assessing the quality of Autonomous Systems. The goal of this thesis is not to define a global or absolute quality rating of Autonomous Systems. The rating is only supposed to be effective and meaningful in a well defined context referred to as the "rating domain". The rating domain consists of a set of end-hosts as well as the connections among them over the Internet. Every AS which is traversed on one of the connections within the rating domain can be rated. The rating is done from the perspective of the rating domain in which measurements are performed and does not suppose to have a global and absolute expressiveness.

The rating system is meant to be applicable for Internet users which use multiple, distributed hosts communicating to each other. Examples are users which make use of distributed computing, grid computing, or VPNs. Furthermore the system must be applicable for users which do not have access to the network infrastructure (routers, switches, etc.), but only to the end-hosts.

\subsection{AS Quality}

The motivation for rating AS's is to be able to find current and/or permanent weak links of Internet connections. Generally speaking, the quality of an Internet connection is the better the faster data is delivered from the source of the connection to its destination. The speed of data delivery basically relies on two things: the network performance of the traversed networks and the physical length of the taken path.

On its way through the Internet, a network packet passes through Autonomous Systems. The quality of an Internet connection therefore relies on the quality of the traversed AS's. According to the above definition of Internet connection quality, AS quality can basically be determined by:

- The network performance of the AS (including its availability)

- The path it selects to route and to forward packets

Furthermore the qualities of the neighboring AS's—-the providers and peers of an AS-also contribute to its own quality, since all packets traversing the AS will be forwarded to one of its 
neighbors. Better upstreams and peerings result in better end-to-end connections in which an AS is involved.

\subsection{Intra-AS vs. Inter-AS Quality}

On the one hand, an Autonomous System routes packets through its own network, on the other hand it forwards packets to other AS's if the destination of a packet is not within the AS itself. Therefore we can say that an AS has two tasks: Route packets through its network and maintain connections to other AS's for being able to forward packets to the rest of the Internet. According to these two tasks, we distinguish two kinds of AS quality: "Intra-AS quality", i.e., the network performance and the selected path within the AS, and "inter-AS quality", i.e., the quality of the neighbors of an AS.

The following two sections discuss methods of measuring candidate indicators of intra-AS as well as inter-AS quality.

\subsection{Intra-AS Quality: Network Performance}

There are different ways to measure and monitor network performance. Chen [5] classifies them into the following categories:

- router-based

- network layer

- IP/ICMP layer

- transport/application layer

Router-based measurements are passive and do not measure performance directly. They make use of data about traffic, whose characteristics are strongly correlated with performance.

Information about performance can be derived from the protocol composition, traffic bursts and average utilization, flow durations, and packet sizes [5]. Router-based performance analysis can, e.g., be made by the use of SNMP or Netflow data. Agreements with the operators of (core) routers are required to get this data. Due to privacy related problems, this is usually difficult if possible at all. Our approach is to develop a framework usable for any Internet user and therefore router-based measurements are no option.

In contrast to router-based measurements, the rest of the above listed methods usually make use of active measurement techniques. Active methods involve the addition of test traffic and can be performed on different layers of the OSI model.

The advantage of network layer measurements over higher layer measurements is that switches can participate. In our context control over switches cannot be assumed and therefore network layer measurements would not have any benefit over measurements on a higher level.

Using the IP/ICMP layer, measurements across the entire Internet are possible and easy to carry out. E.g., repeated pings are an easy way to obtain a sample distribution function of round-trip time and an estimate of packet loss ratio. The advantage of IP/ICMP measurements is that there 
are numerous tools available and applicable from any end-host. However, IP/ICMP layer measurements have their limitations. Using ICMP one has to be aware that some networks give low priority or even block pings. And with traceroute, a returned ICMP message may not follow the same path as the traceroute packet that triggered it.

Transport/application layer measurements are closer to the application perspective. The basic idea is to run a program emulating a particular application that produces test traffic. However, this only enables end-to-end measurements between two application end points and therefore the analysis of end-to-end connections. The evaluation of intermediate parts of the connection pertaining to a certain AS are not possible.

\subsubsection{IP Performance}

For our approach IP layer measurements are the best possible solution to measure network performance, because IP layer measurements can easily be carried out by any Internet user and-performed appropriately-deliver the required data to rate the network performance of Autonomous Systems. To prevent the afore mentioned problems of ICMP, IP layer measurements can be made using other higher-layer protocols such as, e.g., TCP.

The IP Performance Metrics Working Group of the Internet Engineering Task Force ${ }^{1}$ defines the following set of standard metrics that can be applied to the quality, performance, and reliability of Internet data delivery [19]:

- connectivity

- one-way delay and loss

- round-trip delay and loss

- delay variation

- loss patterns

- packet reordering

- bulk transport capacity

- link bandwidth capacity

\footnotetext{
${ }^{1}$ The Internet Engineering Task Force. http://www. ietf.org/ last visit August 2008
} 


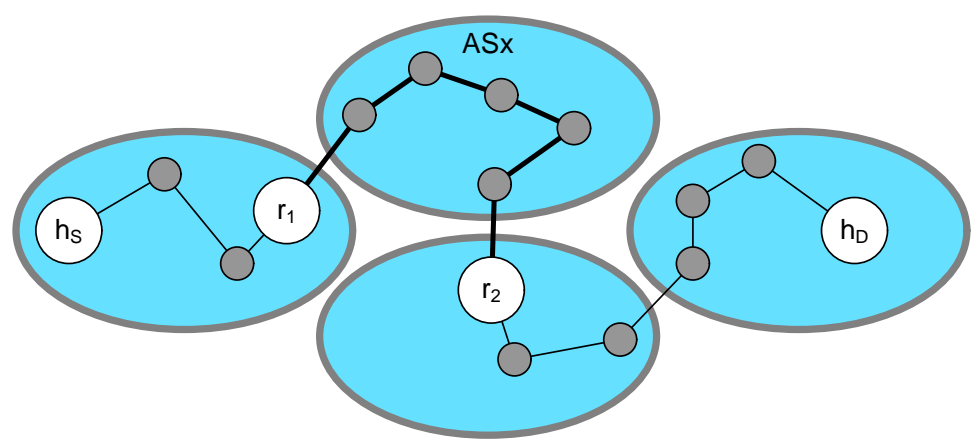

Figure 3.1: Sub path of an end-to-end connection traversing $\mathrm{AS}_{\mathrm{x}}$

These metrics can be applied to indicate the quality of a network path in the Internet. They are measured on end-to-end connections which usually traverse multiple AS's. Examining the sub path of an end-to-end measurement which relates to one specific AS, they are candidate indicators for the quality of the Autonomous System in terms of network performance. Figure 3.1 illustrates a sub path of an end-to-end measurement traversing $\mathrm{AS}_{\mathrm{x}}$. The metrics examined on the path between $r_{1}$ and $r_{2}$ relate to the quality of $A S_{x}$. Notice that in most cases measurements cannot be performed on $r_{1}$ and $r_{2}$ since they are not end-hosts but intermediate routers-as in Fig. 3.1-which are not under our control (cf. first paragraph of this chapter).

Related to the quality of an AS, the metrics have the following meanings:

Measure

connectivity

one-way loss

round-trip loss

loss patterns

one-way delay round-trip delay delay variation packet reordering

bulk transport capacity

\section{Description}

does the AS provide a connection between host $r_{1}$ and host $r_{2}$ how many packets are lost between $r_{1}$ and $r_{2}$ on a path traversing the AS in one direction $\left(r_{1} \rightarrow r_{2}\right)$

how many packets are lost between $r_{1}$ and $r_{2}$ on a path traversing the AS back and forth $\left(r_{1} \leftrightharpoons r_{2}\right)$

are there certain patterns on when and on how many packets are lost on a path through the AS (e.g., packet loss bursts)

how long does it take for a packet to traverse the AS in one direction how long does it take for a packet to traverse the AS back and forth how stable are the delays

how often and how strong are packets of a connection reordered on their way through the AS

what is the maximum throughput obtainable by a single TCP connection going through the AS

link bandwidth capacity what is the available bandwidth on a path through the AS

Special care has to be taken with the performance of connections between Autonomous Systems, i.e., between two routers not belonging to the same AS. Performance problems observed on these particular links influence the quality of both AS's involved. 


\section{Feasible Measurements}

Not all of the afore mentioned IP performance measurements are feasible in the context of this thesis. Our measurements can only be performed and connections can only be established between end-hosts. However, the interesting results are the values measured on sub paths of end-to-end connections, namely on the parts within the different AS's. Figure 3.11 illustrates an example of the situation: Measurements can be performed on the hosts $h_{S}$ and $h_{D}$, but the relevant results to rate the Autonomous System $\mathrm{AS}_{\mathrm{x}}$ are the values measured on the path between $r_{1}$ and $r_{2}$.

Therefore the following metrics cannot be applied: one-way loss, one-way delay, packet reordering, bulk transport capacity, and bandwidth capacity. All of those measures would involve accessing hosts in-between the end-hosts for being able to get the results of the hops on the sub paths. Therefore the feasible measures are reduced to connectivity, round-trip delay, and round-trip loss. Connectivity can be expressed by the packet loss: If all packets of a connection are lost, then no connectivity is available. Thus the network performance of an AS is determined by the round-trip packet delay and the round-trip packet loss within the Autonomous System.

If both end-hosts are owned-as it is the case in our context-then measurements can be done from both sides for the same end-to-end connection. The corresponding measurements can be correlated to make a more accurate statement about the qualities of the traversed AS's.

\subsubsection{Internal Path}

Besides the network performance the internal path contributes to the quality of an AS, e.g., a longer path might be worse than a shorter one. The internal path is the IP path an AS selects to route a packet through its network. It can be determined by inspecting the IP addresses of all hosts the packet passes on its way through the AS.

\subsection{Inter-AS Quality: Peering}

An Autonomous System can only influence its own network performance. However, the path a packet takes after leaving an AS can still indirectly be influenced by the Autonomous System. On the one hand, the AS can decide to which other AS's it wants to maintain connections. On the other hand, it can decide where to forward a packet to if it has several possibilities. These decisions influence the end-to-end paths of Internet connections traversing the AS and therefore contribute to the quality of the AS.

According to Malibago [14], AS-route changes can in some cases be correlated with significant performance changes. Therefore the first candidate indicator for inter-AS quality is the outgoing AS-path a packet takes to reach its destination after leaving the AS. The AS-path a packet takes when it leaves an AS can be determined by inspecting the IP addresses of all hosts on the path and then mapping the IP addresses to the AS they belong to.

\subsubsection{Uplink and Peer Qualities}

The qualities of the providers and peers of an AS contribute to the own quality of an AS since better uplink and peering quality results in better performance on routes traversing the AS. 
Peering information can be queried from various data collections. Examples of publicly available up-to-date repositories are the Internet Topology Collection from UCLA Internet Research Lab ${ }^{2}$ and the CAIDA AS-relationship data collection ${ }^{3}$. Using the peering information the peers and providers of an AS can be determined and their quality ratings included in the rating of the AS.

\subsection{Summary}

After considering various candidate indicators for intra-AS as well as inter-AS quality, we summarize the final indicators to be used for the AS rating:

- round-trip packet delay

- round-trip packet loss

- internal path length

- outgoing AS-path

- uplink and peer qualities

\footnotetext{
${ }^{2}$ Internet Topology Collection, Internet Research Lab, UCLA. http://irl.cs.ucla.edu/topology/. last visit August 2008

${ }^{3}$ AS relationship information data, Cooperative Association for Internet Data Analysis (CAIDA). http://as-rank. caida.org/data/. last visit August 2008
} 


\section{Chapter 4}

\section{AS Quality Rating}

This chapter explains how the quality indicators discussed in the previous chapter are measured and used to actually rate an Autonomous System. The first section describes how measurements deliver the values required for the rating, and how the relevant information for each AS is extracted from the measurement data. After this, the following sections discuss how the measurement data is used to compute quality measures for the actual rating.

\subsection{Measurements}

To measure the quality indicators listed at the end of the previous chapter, we establish connections ${ }^{1}$ passing through the AS's of our rating domain. On these connections-referred to as "measurement connections" - we determine for each traversed AS the following values:

AS-RTT The round-trip packet delay is measured in form of the "AS-RTT". The AS-RTT represents the time a packet spends within the AS. It is determined by subtracting the RTT to the first host within the AS from the RTT to the last host within it. Figure 4.1 shows a connection passing through an AS and the corresponding hosts whose RTTs are used to determine its AS-RTT.

Packet Loss The round-trip packet loss is determined by counting how many packets of the measurement connection are lost within the AS. Two different kinds of lost packets are distinguished. Packets which are lost within an AS, i.e., on a hop between two hosts of the same AS determines the "internal loss". And packets which are lost on a hop between two hosts of different AS's determine the "border loss". Border losses are charged to both AS's of the hosts connected by the hop on which the packet was lost.

Internal Path The first metric concerning the internal path is the number of hosts within the AS on the measurement connection. Furthermore the internal path length is measured by

\footnotetext{
${ }^{1}$ As described in Sect. 5.1.3. we used traceroute to perform the measurements. Therefore we did not establish connections in the sense of a TCP connection, but rather sent single network packets.
} 


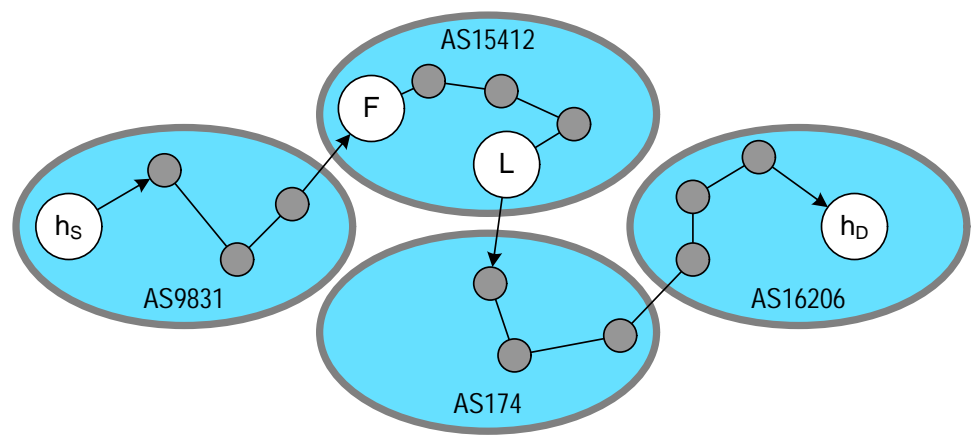

Figure 4.1: The AS-RTT of AS15412 is defined as the RTT to the first host in the AS (F) subtracted from the RTT to the last host in AS15412 (L)

identifying the IPs of all hosts within the AS. After determining the geographical location of the IPs, the distances of all hops within the AS are summed up and result in the internal path length.

Outgoing AS-Path The AS-path from the AS to the destination of the measurement connection is determined by identifying the IPs of the hosts between the AS and the destination and then mapping the IPs to the AS they belong to. In the example shown in Fig. 4.1 the outgoing AS-path of AS15412 is "174-16206".

Uplink and Peer Qualities There are two possibilities to determine the uplinks and peers of an AS. Either by querying a public database holding information about peerings, or by recording the AS's to which an AS forwards the packets on the measurement connections traversing through the AS. While the first method reveals the complete set of neighbors of an AS, the second one only determines the relevant neighbors of the AS within the rating domain. On the example measurement connection shown in Fig. 4.1.AS174 would be recorded as neighbor of AS15412, since AS15412 forwards the packets of the connection to AS174. Furthermore AS15412 is the neighbor of AS9831, and AS16206 the neighbor of AS174.

\subsubsection{AS Measurements}

The results of a measurement connection which belong to a specific AS are referred to as "AS measurement". E.g., the measurement connection shown in Fig. 4.1 delivers AS measurements for AS9831, AS15412, AS174, and AS16206. Each AS measurement contains measurements of all values discussed above.

\subsection{Rating}

To collect data required for the AS rating, many different measurement connections are initiated, traversing all AS's of our rating domain from different "perspectives". They result in various AS measurements for each AS. To rate an AS, the results of all its AS measurements have to be 
evaluated to derive the actual rating. This section discusses methods to derive the rating of the AS's from the relevant measurement data.

\subsubsection{Data Mining}

Our first idea for an approach to process the measured values to derive the rating was to apply Data Mining. The WordNet of Princeton University defines Data Mining as "data processing using sophisticated data search capabilities and statistical algorithms to discover patterns and correlations in large preexisting databases; a way to discover new meaning in data" [20]. Data Mining assumes a large given set of data instances from which it tries to extract new information. The main tasks of Data Mining are:

Classification If the given set is pre-labeled, i.e., every instance of the set is known to belong to a certain class, then classification can be applied to specify a set of rules which can be used to predict the classes of new instances.

Clustering If the given set is not pre-labeled and the instances want to be grouped, then clustering can be applied. Clustering tries to find a natural grouping by clustering instances which are "close" to each other.

Association By searching properties which frequently occur together, Data Mining can create association models.

One could try to apply Data Mining to the collected AS measurement data and derive the rating from there. However, the basic Data Mining approach does not apply to our task. Data Mining tries to extract new, previously unknown information from a large set of data. We have a limited and manageable variety of information. Rather than find new meanings of our data we want to derive correlations between the $\mathrm{AS}^{\prime} \mathrm{s}$ and their measurement data and correlations among the data of the various AS's. This task is better solved by applying and evaluating statistical measures to the measurement data.

Furthermore our approach is meant to be applicable in a practical environment. In order to apply Data Mining to the measurement data for the AS rating, we would have to store all measurement results and continuously process the data to keep the rating up-to-date. This, however, is not feasible due to scalability issues. A growing amount of measurement points would enhance the time needed to process the data dramatically.

To reduce the amount of data to be processed, our framework must somehow pre-process the data before storing it in the database. This, however, is only possible if we know what data is relevant for the rating, i.e., how to reduce the data to a minimum. Once reduced, no Data Mining method would be able to find any new information, since the information is already reduced to what we define to be interesting. Therefore Data Mining is not a feasible method to be used to rate Autonomous Systems. 


\section{Distributed Data Mining}

Distributed Data Mining (DDM $)^{2}$ is an approach to address the scalability issues of a Data Mining solution. DDM makes use of distributed resources. Using DDM for the AS rating, only a small amount of data would have to be exchanged while the Data Mining process is performed locally on the local sensor data. It therefore scales for a large amount of measurement points if they are all involved in the mining process. However, before applying complex methods like DDM, a thorough understanding of the measurement process and potential side effects is necessary. Furthermore, one should look for alternatives first. Both of these steps are the main focus of our thesis. The application and evaluation of DDM is left to future work.

\subsubsection{Comparing Quality Measures}

Since Data Mining does not fit the context of this thesis, we use a statistical approach to derive the rating from the measurement data. Once the definite rating is defined we will be able to set up a method to reduce the collected data and therefore guarantee scalability in terms of the persistent data amount and processing time of the data.

As already mentioned in the last chapter, the rating of the Autonomous Systems is done within a well defined rating domain. The rating of an AS reflects its quality with respect to this rating domain. We define the quality measures as statistical values based on the metrics discussed in Sect. 4.1 These quality measures can be computed for each AS; comparing them among the AS's allows us to derive the rating.

For the long-term analysis, we compare the values of an AS to all other AS's of the rating domain. Thus the long-term rating states how well an AS performs compared to the other AS's of the same context.

The real-time rating of an Autonomous System compares the AS to other AS's as well as to the historic values of its own measurement data. I.e., besides comparing it to other AS's, the real-time rating also highlights how well an AS performs with respect to its own "usual" performance. This is achieved by comparing the results of a real-time AS measurement of a specific AS on a specific connection to the historic values of the same setting.

\subsection{Statistical Quality Measures}

After describing the AS measurements and defining the approach to derive the rating, this section discusses the required quality measures for the statistical rating approach.

Each metric described in Sect. 4.1 is measured on each AS-measurement. The following subsections describe how the results of all AS-measurements of an AS are combined to derive a single value as quality measure.

\subsubsection{Delay Stability}

The first quality measure of an AS is the stability of its AS-RTT. The smaller the variation of the AS-RTT the higher its stability the better the quality of the AS. The variation of the AS-RTT,

\footnotetext{
${ }^{2}$ Refer to http://www.cs. umbc. edu/ hillol/DKD/ddm_research.html last visit August 2008
} 

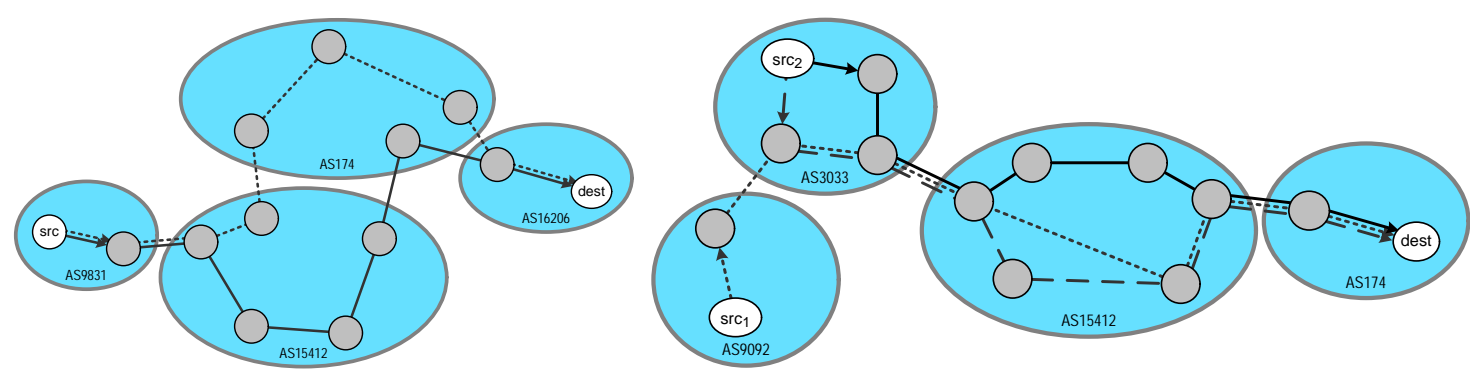

Figure 4.2: Left side: Two end-to-end measurements whose values of AS15412 are not comparable. Right side: Three measurements whose values of AS15412 are comparable.

however, cannot be computed from the complete set of AS-measurements of an AS. Some measurement connections only stripe an AS whereas others traverse it over hundreds of kilometers. Therefore we group the AS measurements into subsets of comparable measurements. For each set of comparable AS measurements we can determine the variation of the AS-RTT.

We define two AS measurements to be comparable, if they enter the AS from the same host and leave the AS to the same host. Only if these conditions hold, two measurements are similar enough to allow the comparison of their AS-RTT. On its left side, Fig. 4.2 shows two measurement connections whose AS measurements of AS15412 are not comparable. The right side shows three measurements whose AS measurements of AS15412 are comparable.

There are different ways to define the variation of a set of values. A simple approach is by computing the coefficient of variation (COV) which is defined as $c_{v}=\sigma / \mu$, where $\sigma$ denotes the standard deviation and $\mu$ the mean of the value set. However, the COV is only useful if the distribution of the values is unimodal and symmetrical. It turned out that this is not the case with RTTs (cf. Sect. 7.1.1).

An other appropriate measure for the variation is the interquartile range (IQR). The IQR is defined as $I Q R=Q_{3}-Q_{1}$, whereas $Q_{i}$ denotes the $i$-th quartile. The IQR does not require any characteristics of the data distribution. Further discussion about the COV and the IQR can be found in Sect. 7.1.1 and 7.1.2.

Using the IQR, we can compute the stability of each set of comparable AS measurements. This results in a set of stability values for each AS. For the rating, however, we require one single value per AS. Therefore we have to combine all stability values of an AS to one single value.

To combine all values of an AS, we must first normalize them. We divide the IQR of each subset of comparable AS measurements by the median AS-RTT of the subset. To finally get one single value for the quality measure, we determine the median of all normalized IQRs of an AS. This value defines the delay stability of an AS and can be compared among all AS's. The lower the overall variation of the AS-RTT the better the quality of the AS.

\subsubsection{Delay per Length}

The delay per length can be computed for each AS measurement by dividing the AS-RTT by the internal length. Since this results in a relative (normalized) value, we can combine the delay per 
length values of each AS measurement of an AS to derive the quality measure. Again we determine the median of all delays per length as the final quality measure to compare it among the AS's. The lower the overall delay per length the higher the quality of the AS.

\subsubsection{Internal Loss}

To derive a quality measure from the internal loss counts of the AS measurements we normalize the loss count of each AS measurement by dividing it by the internal path length. From all normalized loss counts we determine the median to compare it among the AS's. The lower this value the better the quality.

\subsubsection{Border Loss}

Border loss is similar to internal loss. However to normalize the values the loss count is divided by the length of the connections to the previous and next AS instead of the internal path length. Again, the lower the value the higher the quality.

\subsubsection{Internal Length Stability}

The quality measure of the internal length is its stability correlated with the delay stability (cf. Subsect. 4.3.1). Unstable internal length combined with high delay stability is good, since it indicates good load balancing. Stable internal length combined with low delay stability is bad, since it indicates bad or no load balancing.

To derive the stability of the internal length we follow the same procedure as for the AS-RTT:

1. Group all AS measurements of an AS into subsets of comparable measurements

2. Divide the IQR of each subset by the median of the subset

3. Determine the median of all these values

The resulting median denotes the overall internal length variation. The variation correlated together with the delay stability can be compared among the AS's.

\subsubsection{Outgoing AS-Path}

The outgoing AS-path is correlated with the end-to-end delay of the respective measurement. Whenever the outgoing AS-path of an AS measurement is different than the one of the previous measurement, and at the same time the RTT from the AS to the destination is higher, then this event is recorded. Such an event suggests, that the change of the AS-path caused the worsening of the RTT. The total number of those events divided by the total number of AS measurements of the AS can be compared among the AS's. The lower this ratio the better the quality of the AS. 


\subsection{GeoIP Accuarcy}

The expressiveness of the two quality measures "Delay per Length" and "Internal Length Stability" highly depends on the accuracy of the coordinates derived from the IPs. The computed length of a path between two locations is only as accurate as the provided coordinates of the two locations. According to MaxMind the accuracy of the free database "GeoLite City" - which we are using - is "over $99.3 \%$ on a country level and $74 \%$ on a city level for the US within a 25 mile radius." Details on the accuracy are described on their hompag $£^{3}$

Being aware of the limit of correctness of the IP coordinates, we will include both measurements. However, their effect on the actual rating will be limited according to the accuracy of the coordinates. I.e., an AS which is located in a country with low accuracy (e.g., in the Republic of Korea with only $16 \%$ in July 2008) the measurements depending on GeoIP won't have any negative influence on the rating.

\subsection{Measurement Problem}

To test and evaluate our rating approach we collected measurement data during a period of 38 days. The data was collected using traceroute tools as further described in Sect. 5.1.3 and 6.1. Before applying our rating approach we thoroughly inspected parts of the data to see whether the approach seems applicable (cf. Sect. 7.1). Unfortunately we made some observations which turned out that our previously described approach is not applicable in the way we defined it.

\subsubsection{Observations}

Delay stability, delay per length, and the outgoing AS-path measure are based on the AS-RTT which is composed from the difference of two round trip times to intermediate hosts of the traceroute output. Therefore they rely on the correctness of the RTTs measured by the traceroute tool. During the analysis of the measurement data we plotted the AS-RTT of different AS's and discovered that for all samples we analyzed, the AS-RTT was often negative. The left side of Fig. 4.3 shows the plot of all AS-RTTs measurement of an AS on a specific end-to-end connection. Obviously the measured RTTs of the proceeding hosts are often not monotonically increasing. The right side of Fig. 4.3 shows the plot of the consecutive RTTs of a sample traceroute measurement.

Analyzing the measurements ${ }^{4}$ using the Perl script fetch_decrease.pl resulted in the following statistics which clearly show that measurements with non-monotonically increasing RTTs are not just individual cases but a regular phenomena:

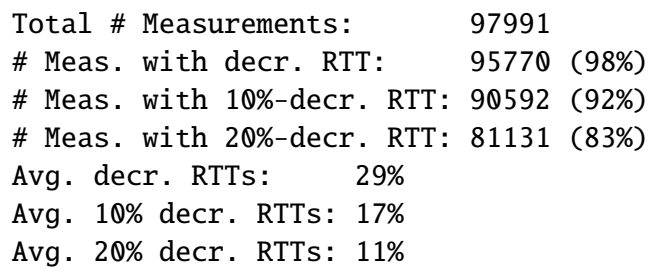

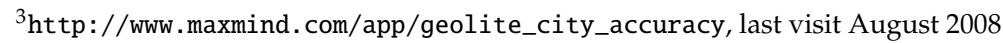

${ }^{4}$ not all measurements performed but the ones which have been stored in the DB at that point in time, namely 97991 

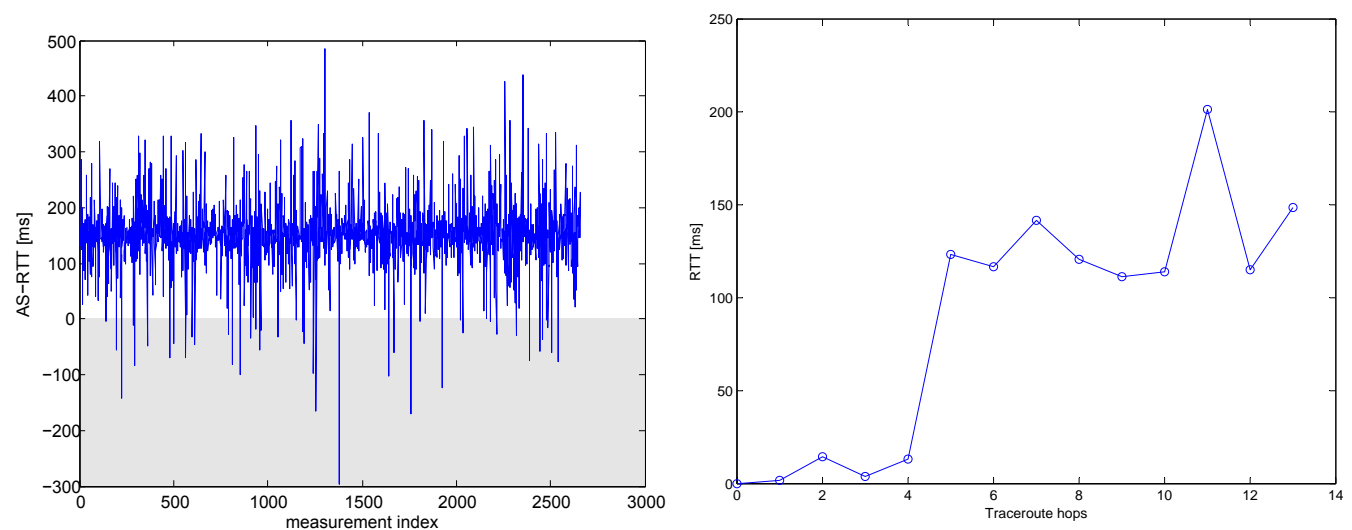

Figure 4.3: Sample AS-RTTs on the left. Sample traceroute RTTs on the right.

98\% of all measurements include at least one hop with decreasing RTT. 92\% include at least one hop with a decrease of at least $10 \%$ of the RTT, and $83 \%$ include at least one hop with a decrease of at least $20 \%$. Further analysis shows that $29 \%$ of all hops are decreasing, $17 \%$ decrease by at least $10 \%$, and $11 \%$ of all hops decrease by at least $20 \%$.

\subsubsection{Possible Causes}

The most likely reason for this effect is because routers are designed to perform in forwarding network packets. Responding the expiration of the TTL of a packet is not of high priority. For a router it is much more important to forward packets of active connections than to report the failure of a packet delivery. Therefore the answering time of a router may vary due to queued answer packets and due to the low priority of reporting expired TTLs.

Another possible reason is that the ICMP return packets of two succeeding hosts can take different routes back to the source. If the route taken by the answer sent from the second host is faster than the one by the first one, then the difference of their RTT can get negative. However, we don't think that routes differ so often and that if they are differing that the difference is often that noticeable.

\subsubsection{Consequences}

Due to the many non-increasing RTT sequences in traceroute measurements, any quality measure which relies on the difference of intermediate RTTs is not expressive and therefore not feasible. I.e., delay stability, delay per length, internal length stability, and outgoing AS-path as described in Sect. 4.3 are no valid quality measures. Generally any intermediate high RTT (as such) is not a reliable indication of a bad performing AS, since the high value could be the result of a slowly answering router. The quality of the AS in routing packets can still be well.

Furthermore individually lost packets are no reliable indicator for the packet loss rate, since the lost packets could be dropped ICMP answers rather than the actual probe packets. I.e., the packet loss measures as defined by us are not feasible as well. 


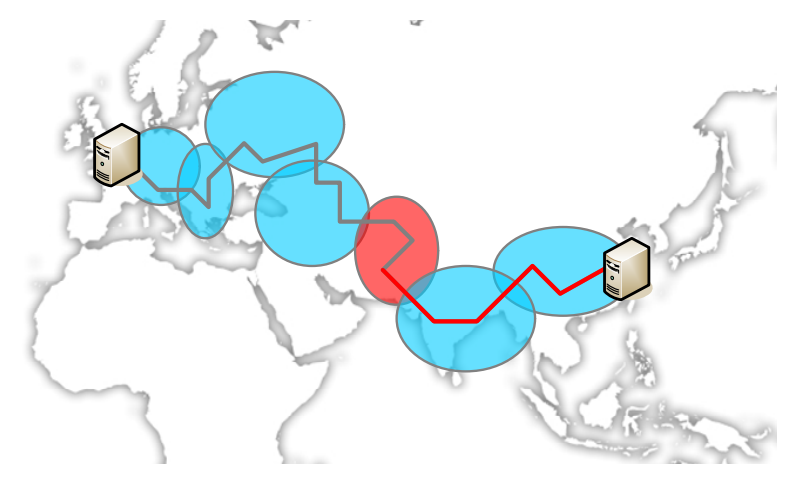

Figure 4.4: End-to-end connection traversing multiple Autonomous Systems slowed down by a bad performing AS

\subsection{New Rating Approaches}

After realizing the problems of our original approach we came up with new ideas to interpret the measurement results.

\subsubsection{Ignore Non-Increasing Traceroute Segments}

Our first idea was to ignore the non-increasing parts of the traceroute results. The major drawback of this idea is that parts of the collected information gets lost. Whenever a non-increasing sequence overlaps the border of two AS's no statement about the quality of the two AS's can be made since their AS-RTT can no longer be calculated. In our measurements, almost a third of all hops are decreasing. Therefore a lot of information would get lost using this approach.

\subsubsection{Outlier-based Measurement Interpretation}

The basic idea of this approach is illustrated in Fig. 4.4 and works as follows: Whenever the end-to-end RTT of a measurement is significantly higher than usual, then the AS which causes the outlying RTT is determined and recorded. Real-time measurements are compared to collected historic data and the AS causing current poor network performance is detected. Furthermore periodic measurements are continuously compared to collected data and whenever an AS causes poor performance it is recorded. The more often an AS causes outlying end-to-end RTTs the worse is its long-term quality.

\section{End-to-End RTT}

If we initiate the measurement connections to destinations which are not routers (but work servers, stations, or gateways), then the round-trip time from the source to its destination-the end-to-end RTT-does not suffer from the problems described in Sect. 4.5. Therefore we can assume that a high end-to-end RTT effectively indicates a slow connection. 


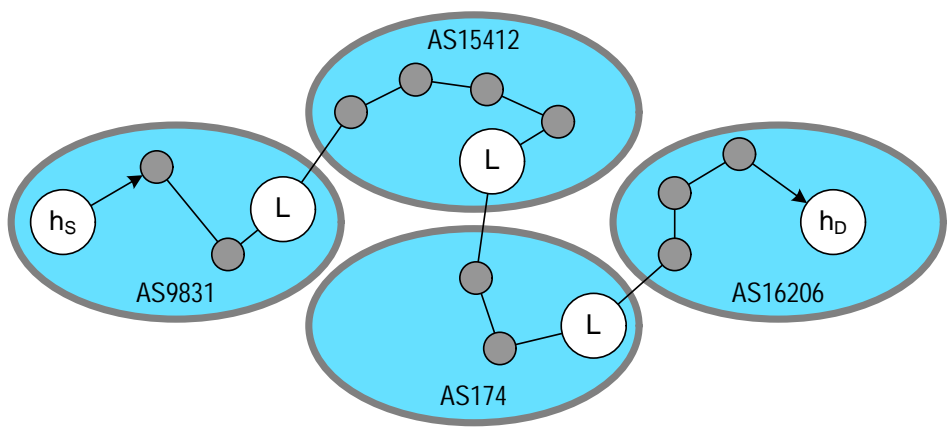

Figure 4.5: The RTT to the last host within an AS (L) determines the AS-end-RTT

\section{Guilty AS}

For every initiated measurement connection, its AS-route is determined. For each particular AS-route of an end-to-end measurement the RTT to the last host of each AS-referred to as the "AS-end-RTT"—is recorded (cf. Fig. 4.5).

Once an outlying end-to-end RTT is observed, each AS-end-RTT is compared to its recorded values to see whether it is also outlying. The guilty AS is the one which has an outlying AS-end-RTT and whose following AS's on the AS-route all have outlying AS-end-RTTs as well.

If the AS-end-RTT of an AS is outlying but one of the following AS on the route does not have an outlying AS-end-RTT, then we can assume that the former outlier is caused by a router which answers slowly but still forwards packets well. Therefore we avoid misinterpretations caused by the traceroute problems discussed in Sect. 4.5 . The following annotated traceroute output illustrates such a scenario:

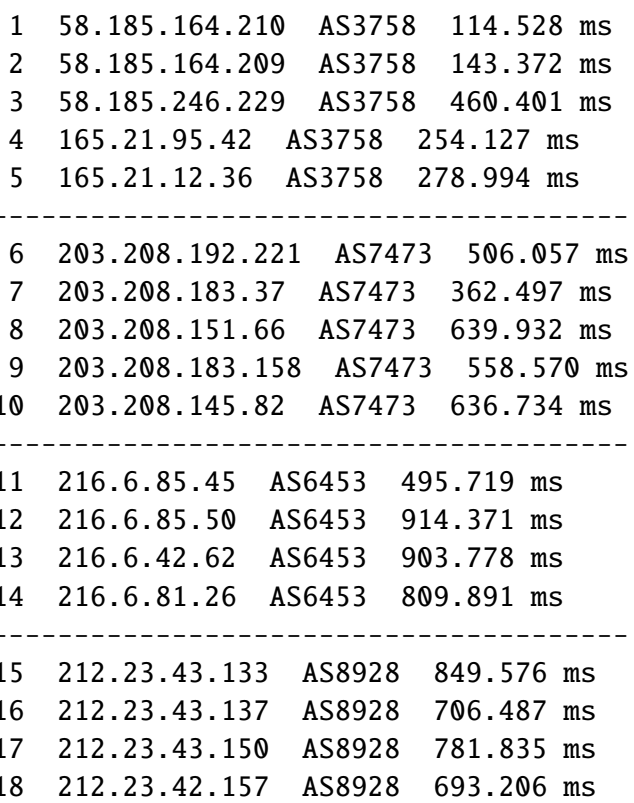




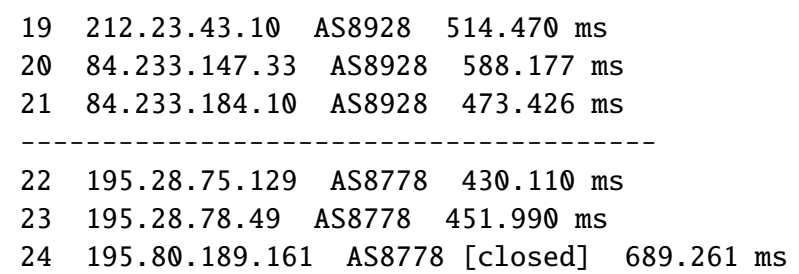

This output is taken from our measurements performed throughout the thesis. According to the outlier definition described in the following subsection, it turned out that the end-to-end RTT of this measurement is outlying. Furthermore we determined, that the AS-end-RTT of AS7374, AS6453, and AS8778 are outlying as well. However, the AS-end-RTT of AS8928 is not outlying.

Therefore neither AS7473, nor AS6453 are blamed to cause the outlying end-to-end RTT, since they still forward packets efficiently to AS8928. The outlying end-to-end RTT is thus assumed to be caused by the very last AS on the route.

\section{Outlier Definition}

A common definition for an outlier is based on the interquartile range. John Tukey defines an outlier as any value which is more than three IQRs below the first or above the third quartile. I.e., an outlier is any value which is not within the range

$$
\left[Q_{1}-3 \times\left(Q_{3}-Q_{1}\right), Q_{3}+3 \times\left(Q_{3}-Q_{1}\right)\right]
$$

whereas $Q_{i}$ denotes the i-th quartile [26] (cf. Sect. 7.1.2 concerning IQR and quartiles). Figure 4.6 illustrates the outlier definition with a sample measurement series.
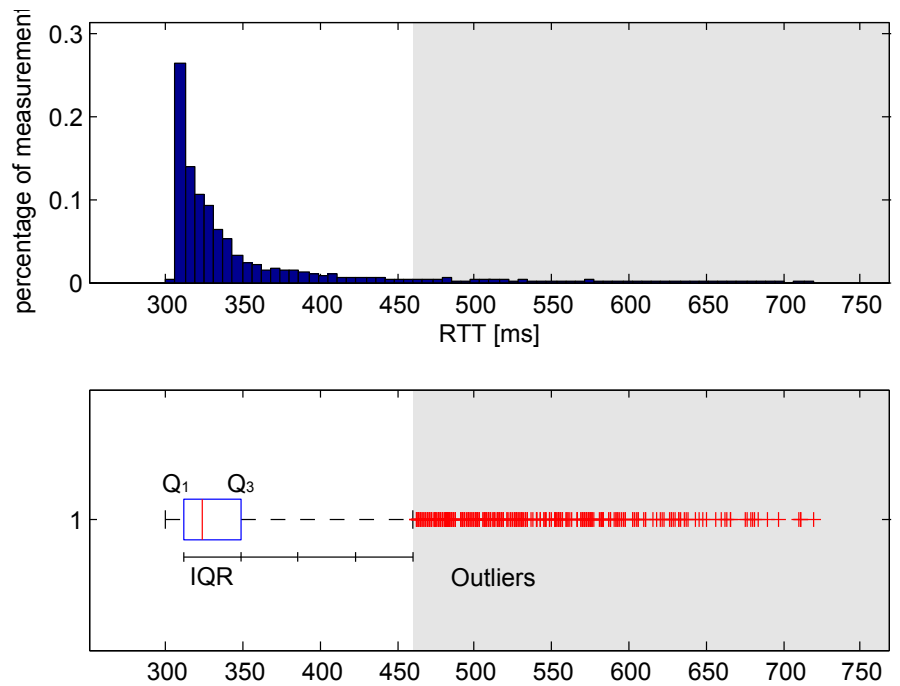

Figure 4.6: Histogram and corresponding boxplot illustrating the outliers of a sample measurement series 


\section{Intra-AS Quality Rating}

Using this new approach, the primary statement we can make about the intra-AS quality of an AS is the ratio between the number of times the AS caused an outlier and the total number of end-to-end measurements that included the AS:

$$
\text { outlier_ratio }\left(A S_{x}\right)=\frac{\# \text { times } A S_{x} \text { caused an outlier }}{\text { total \# measurements including } A S_{x}}
$$

The lower the outlier-ratio the better the quality of the AS. However, one must consider that the reliability of the outlier-ratio depends on the number of measurements including the AS. An AS causing one outlier in a thousand measurements might not have the same quality as one which causes ten outliers in ten thousand measurements. Comparing two AS's, it is therefore important to not only compare the values of the outlier-ratios but also their reliabilities.

Furthermore we investigate the connectivity of the AS. The corresponding quality measure is the ratio between the number of times an AS interrupts a connection and the total number of measurements the AS is included:

$$
\text { connectivity_ratio }\left(A S_{x}\right)=\frac{\# \text { times } A S_{x} \text { interrupted a connection }}{\text { total \# measurements including } A S_{x}}
$$

Whenever a measurement does not reach its destination, the last AS on the observed AS-route is assumed to be the one causing the interruption. The lower the connectivity-ratio the better the quality.

To get the final intra-AS rating, the outlier-ratio and the connectivity-ratio are combined to a single measure. Depending on the application domain of the rating, delay and connectivity are of different importance. Therefore a weighted sum of the two ratios delivers an appropriate quality measure according to the importance of the two factors:

$$
\text { intra_AS_quality }\left(A S_{x}\right)=\alpha * \text { outlier_ratio }\left(A S_{x}\right)+\beta * \text { connectivity_ratio }\left(A S_{x}\right)
$$

\section{Inter-AS Quality Rating}

The inter-AS quality is determined by analyzing the qualities of the neighbors of an AS as described at the end of Sect. 4.1. The concrete formula to compute the inter-AS quality is show in 4.5):

$$
\begin{array}{r}
\text { inter_AS_quality }\left(A S_{x}\right)=\sum_{A S_{i} \in N} \text { weight }\left(A S_{i}\right) * \text { intra_AS_quality }\left(A S_{i}\right) \\
\text { where } N=\left\{\text { all neighbors of } A S_{x}\right\}
\end{array}
$$

\section{Long-Term Rating}

The above mentioned quality measures represent the long-term quality of the AS's. They are computed from the results of all measurements performed during a certain period of time and represent the quality of the AS's over this period of time. 


\section{Real-Time Rating}

With our new approach a real-time rating is not possible. Observing connection problems we can detect the guilty AS in real-time. However, a more accurate rating than "guilty vs. not guilty" is not possible with the new approach. 


\section{Chapter 5}

\section{Specification of the Framework}

This chapter specifies the framework developed to perform the measurements and the rating.

\subsection{Data Collection}

According to the quality rating discussed in the previous chapter, the following concrete information has to be gathered on each end-to-end measurement:

- IP addresses of all hosts between the source and the destination

- For each IP address the ASN of the AS it belongs to

- Round-trip time to each host on the path

\subsubsection{Measurement Points}

The AS rating is valid within the rating domain consisting of a set of end-hosts as well as the connections among them over the Internet (cf. beginning of Chap. 3). Every host of the rating domain is a measurement point, i.e., measurements for the rating are performed from each host to every other host. All AS's traversed by the connections are included in the measurements, most of them multiple times on different end-to-end connections resulting in many different measurement perspectives.

\subsubsection{Configurations}

To be able to find out whether Autonomous Systems perform differently with different kinds of packets we perform each measurement multiple times with different configurations.

We use two different protocols, namely ICMP and TCP on port 80. ICMP is one of the core protocols of the Internet protocol suite and the standard protocol for network measurements. TCP on port 80 is used by HTTP, the protocol used for data exchange in the World Wide Web. 
Since most traffic on the Internet uses HTTP, we assume that packets using TCP on port 80 are the packets which are treated least exceptionally by routers in the Internet.

Furthermore we use two different sizes of probe packets. The minimal size of a packet, i.e., a packet with an empty payload. And the maximal size of a packet, i.e., a packet with a payload such that the complete size of the packet equals the maximum transmission unit (MTU).

\subsubsection{Network Measurement Tools}

To measure the data specified at the beginning of this section a traceroute-alike too ${ }^{1}$ is sufficient. There are several different implementations of traceroute. Some examples of UNIX implementations are:

- traceroute, the original version of the tool

- mtr, combines traceroute and ping in an interactive command-line tool

- GTrace, a graphical version visualizing routes on a map

- tcptraceroute, TCP traceroute version

- paris-traceroute, avoids traceroute anomalies caused by load balancing

We decided to use paris-tracerout $\mathrm{e}^{2}$ as well as tcptracerout $3^{3}$ for our measurements. Both tools are able to avoid anomalies caused by routers performing load balancing as described by Augustin et al. in [8]. The other traceroute implementations are not capable of avoiding such anomalies.

paris-traceroute would be able to use the TCP protocol. However, we recognized some problems during the tests of the tool using TCP. Therefore we decided to use paris-traceroute only for the ICMP measurement and to additionally use tcptraceroute for the TCP protocol.

\section{Number of Probes}

Per default traceroute sends a batch of three packets per TTL value. We only send one probe per TTL value. Sending only one probe safes time and therefore keeps the amount of time required for a complete set of end-to-end measurements low. This is important for the reason of scalability.

\section{ASN Determination}

paris-traceroute as well as tcptraceroute do not determine the AS to which the hosts belong. Therefore we have to determine the AS of each host subsequently when processing the output files. To get the AS of a given IP we use the RISwhois service of RIPE4 The service can be

\footnotetext{
${ }^{1}$ See http://wikipedia.org/wiki/Traceroute for further details about traceroute (last visit August 2008)

${ }^{2}$ Paris-traceroute, a new version of the well-known network diagnosis and measurement tool. http://www. paris-traceroute.net/. last visit August 2008

${ }^{3}$ Tcptraceroute, a traceroute implementation using TCP packets. http://michael.toren. net/code/tcptraceroute/. last visit August 2008

${ }^{4}$ cf. http://www.ripe.net/projects/ris/tools/riswhois.html for details about the service (last visit August 2008)
} 


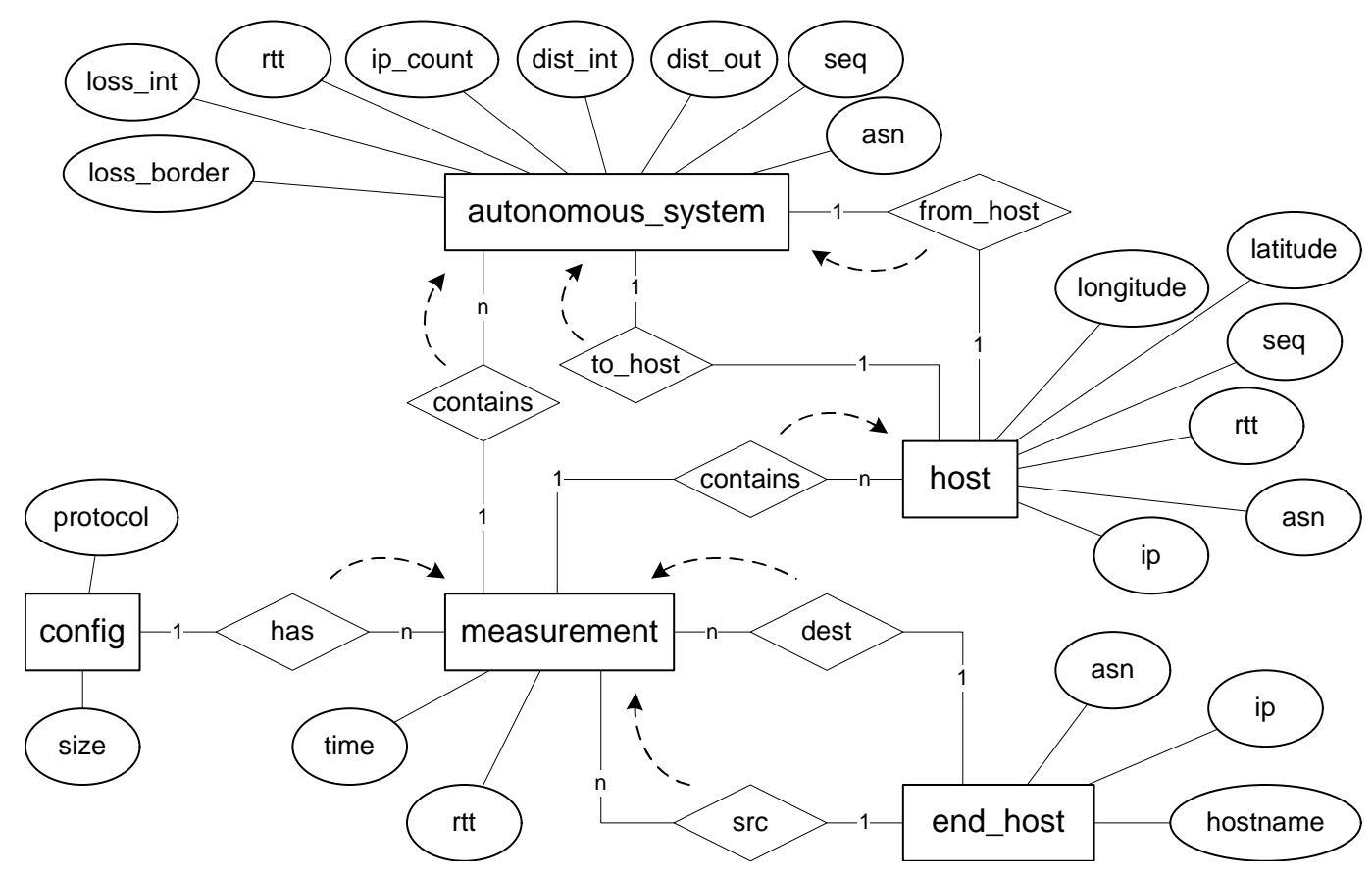

Figure 5.1: ER-scheme of the initial database

accessed by querying the RISwhois server using the whois tool by executing the following command:

whois -h riswhois.ripe.net 123.45.67.89

\subsection{Storage}

\subsubsection{Initial Approach}

In order to be as flexible as possible for analyzing the data, we developed a database scheme which stores all available information of the traceroute outputs. The complete ER-scheme of the database can be seen in Fig. 5.1 .

Table "end_host" contains all measurement points of the rating domain. The configuration details of each measurement are stored in table "config".

For every performed measurement a record in table "measurement" is created. Each measurement record is linked to a configuration in table "config", and to its source and destination stored in table "end_host".

The results of each host of the measurement connection are stored in table "host". For every host of every measurement an own record is created. The records are linked to the measurement they belong to in the "measurement" table. 


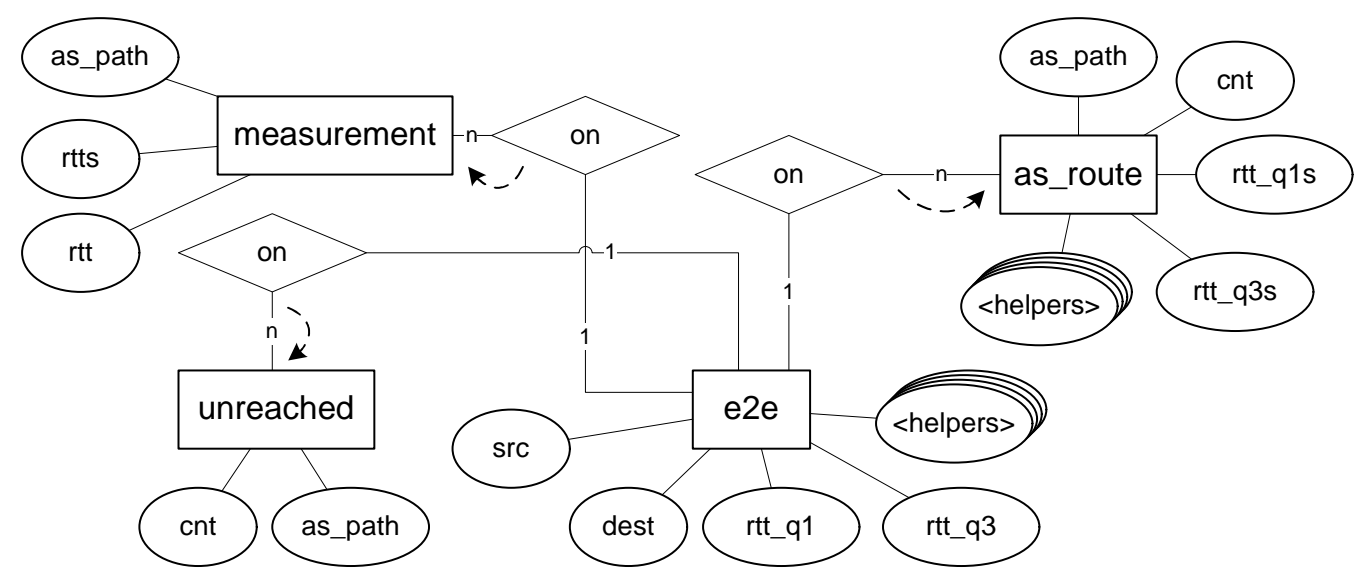

Figure 5.2: ER-scheme of the new database

Furthermore for every AS on the AS-route of the measurement a record in table "autonomous_system" is created containing the results of the AS measurement.

The meaning of each table and its fields is described in more detail in Appendix A.1.

\subsubsection{New Approach}

Because of the poor performance of filling the database using the initial database scheme we developed a new scheme (cf. Sect. 6.2.1 regarding the performance issues). The new scheme not only addresses the performance issues but is also specific to the new rating approach. I.e., it makes the data analysis less flexible but much more efficient.

The scheme contains less information, namely less tables and less foreign key constraints than the original scheme. The lighter and less complicated structure improves the performance when inserting data records, since fewer and less complicated statements are required. In addition information gets preprocessed before entering into the database. Therefore the number of records is dramatically reduced.

The ER-scheme of the new database is shown in Fig. 5.2. The meaning of each table and its fields is described in Appendix A.2.

Each end-to-end connection of the rating domain-i.e., each connection on which measurements are performed-is stored in table " $\mathrm{e} 2 \mathrm{e}$ ". The records of this table contain the source and destination of the connection as well as the first and third quartile of the end-to-end RTT measured on this connection.

Table "as_route" stores a record for every AS-route observed on each end-to-end measurement. The records contain the quartiles of the AS-end-RTTs of all AS's on the AS-route.

The RTT results of measurements which reach their destination are used to update the quartiles of the corresponding "e2e" and "as_route" records. For measurements which did not reach their destination a record in table "unreached" is created. 


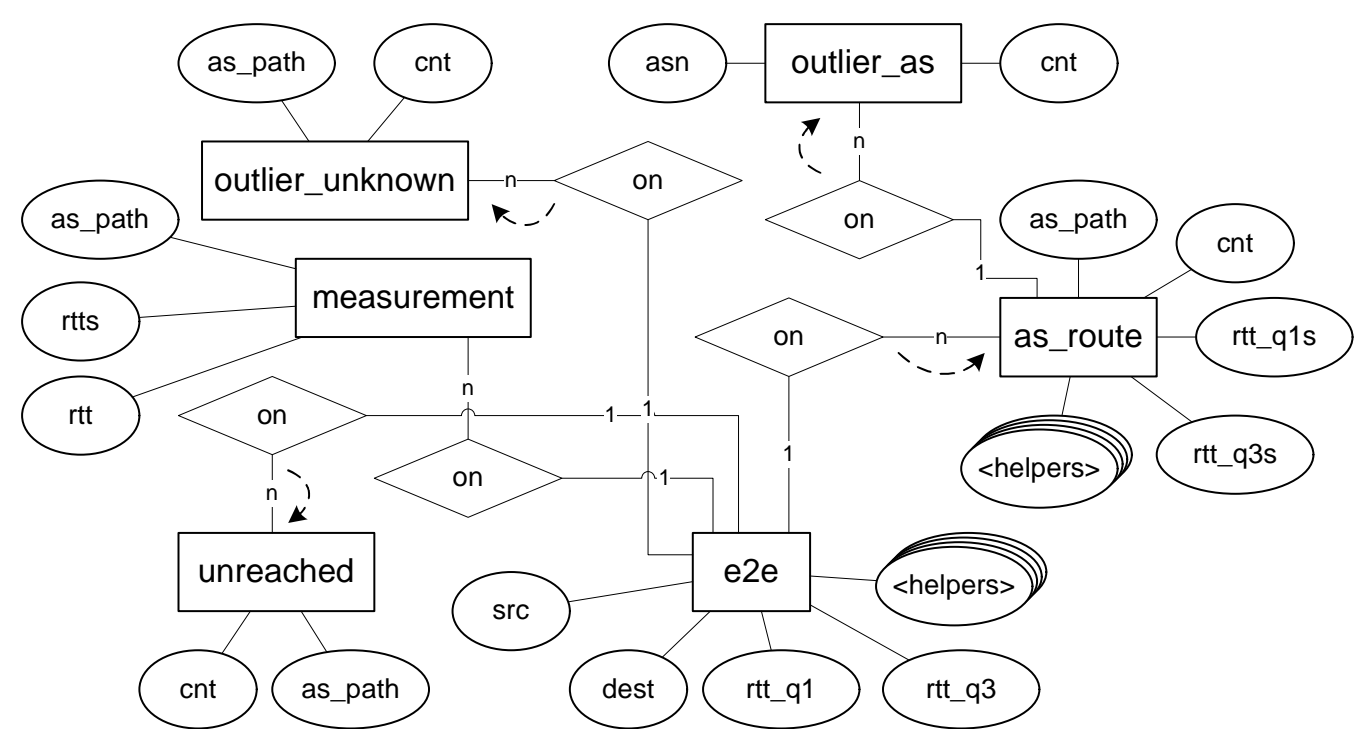

Figure 5.3: ER-scheme of the extended database

\subsection{Rating}

The actual rating is performed on the data of the central database which we described in the previous section. For every measurement we determine whether it has an outlying end-to-end RTT according to the outlier definition described in Sect. 4.6.2 If yes, the same check is performed for each AS-end-RTT to determine the AS causing the bad performance.

If no AS can be determined to be guilty, then the outlier is recorded as "unknown". These outliers occur, if an AS-route is in general slower than the other AS-routes on the same end-to-end connection. On a slow AS-route the end-AS-RTTs will not be outlying while the end-to-end RTT is an outlier with respect to all measurements of the end-to-end connection.

To store the results of the analysis we extend the database by two new tables. One holding the outliers caused by a bad performing AS (table "outlier_as") and one to store the outliers for which no AS was found guilty (table "outlier_unknown"). The ER-diagram of the updated database is shown in Fig. 5.3. The meaning of each table and its fields is described in Appendix A.3. 


\section{Chapter 6}

\section{Implementation}

This chapter describes the implementation of the framework specified in the previous chapter. The source code developed for the implementation can be downloaded from the Internet ${ }^{11}$ The results of the evaluation of the measurement data is discussed in the following chapter.

\subsection{Data Collection}

To perform the measurements we developed the Perl script measured.pl. The script runs as a daemon in the background of all measurement points and periodically invokes the traceroute tools to every destination using each configuration described in Sect. 5.1.2 The calls are made in parallel. paris-traceroute offers the functionality to traceroute to multiple destinations in parallel using multiple threads. tcptraceroute does not offer this option. To have multiple tcptraceroute invocations at the same time the script forks multiple tcptraceroute child processes. However, the concurrency-i.e. the number of threads and concurrent children-is limited to make sure that the CPU consumption is kept low. This is necessary since the framework is supposed to work on productive hosts and must not noticeably influence ongoing processes.

The measurement results, namely the output of the traceroute tools, are stored as raw data in plain text files.

\subsubsection{Measurement Intervals}

According to RFC 2330 [10], periodic measurements at a constant frequency suffers from two potential problems: First, if the metric being measured itself exhibits periodic behavior, then there is a possibility that the sampling will observe only part of the periodic behavior if the periods happen to agree. And second, the act of measurement can perturb what is being measured, and repeated periodic perturbations can drive a network into a state of synchronization.

The intervals between two measurements therefore have to be irregular; the arrival of new

${ }_{1}^{1}$ http://laurent.zimmer.li/code/rating_as_code.tar.gz 
samples must not be predictable. According to PASTA (cf. [27]) this is best achieved by "Poisson Sampling" which uses an exponential distribution to create the intervals. However, there are two reasons why we use uniformly random distributed intervals instead of Poisson Sampling. First, is due to practical reasons: The measurement process itself requires a certain amount of time. Poisson Sampling can result in an interval which is shorter than the measurement time itself resulting in parallel or "dropped" measurements. Furthermore Baccelli et al. [9] showed that if "rare probing" is used, then Poisson Sampling does not bring any advantage over uniformly distributed intervals.

In our script the intervals between two proceeding measurements are uniformly random distributed between 10 and 20 minutes.

\subsection{Database}

For the data repository we used a PostgreSQL database on a Linux work station running the Linux distribution Ubuntu. To create and query the database and its tables we made use of the pgAdmin tool.

\subsubsection{Filling the Initial Database}

To fill the database with our measurement data we compressed the data files of all measurement points into data archives and downloaded them to the work station hosting the central data repository.

We wrote the Perl script store_data.pl to process the data files. The script first checks the validity of the data archive and extracts the archive if it is not corrupted. Then it parses all files containing the raw traceroute outputs. To determine the AS to which a host belongs, it queries the riswhois server as described in Sect. 5.1.3 To get the coordinates of a host, the script accesses the GeoIP database which we downloaded to the work station that runs the scrip $t^{2}$ The distance between the hosts is computed using the formula of Vincenty. The rest of the required information can be parsed from the raw output. A logging mechanisms logs important events and errors during the parsing procedure in log files.

Using the initial database scheme (cf. Fig. 5.1) we soon realized that the performance of filling the database was very poor. Due to the foreign key constraints multiple select statements had to be done before every insertion. Furthermore the number of records grew very fast and slowed down the performance dramatically.

\subsubsection{Filling the New Database}

After the development of our new approach we designed a new database scheme (cf. Fig. 5.2) specific to the new approach and more light-weight to improve the performance of filling data into it. To fill the new database we wrote the Perl script store_data_e2e.pl. The filling procedure of the new database performed much better.

\footnotetext{
${ }^{2}$ The free GeoIP database can be downloaded from http://www.maxmind.com/app/geolitecity (last visit August 2008)
} 
The new script does not require the coordinates of and distances between the IP hosts. However, it has to determine the quartiles of all end-to-end RTTs as well as all AS-end-RTTs. To compute the quartiles without storing each measurement value, the script applies the $\mathrm{P}^{2}$ algorithm for dynamic calculation of quantiles and histograms without storing observations developed by Jain et al. [28].

Even though our script to fill the new database performed much better than the initial one, we were still not able to store all measurement data in the database. store_data_e2e.pl stored the data of about four archives into the database per day. Since we were only able to spend a bit more than a week of time to fill the database, we only stored about $20 \%$ of the measurement data into the database, namely about 16 million traceroute measurements performed from 31 source hosts to 159 destinations.

\subsection{Data Evaluation}

\subsubsection{Initial Tests}

For some initial tests on the measurement data (cf. Sect.7.1) we developed the Perl scripts fetch_triples.pl, fetch_e2e.pl, fetch_outgoing.pl, and fetch_losses.pl to fetch the desired data from the database and write it to MATLAB files to analyze the data in MATLAB.

\subsubsection{Rating}

To evaluate the data and make the AS rating we developed another Perl script. evaluate_e2e.pl performs the rating of the AS's as specified in Sect. 5.3. and stores the results in the database. 


\section{Chapter 7}

\section{Evaluation}

This chapter discusses the evaluation of the measurement data and the application of our rating approach.

\subsection{Initial Tests}

To test our initial rating approach we performed some tests on parts of the data to see whether the approach is applicable. These tests were done on the initial database and only on a small portion of the measurement data.

\subsubsection{RTT Distribution}

First we examined the probability distribution of the RTT. Knowing the probability distribution of the RTT we can determine what measure is appropriate to express the delay stability, namely whether we better use the coefficient of variation (COV) or the interquartile range (IQR) as indicated in Sect. 4.3.1

To get a first impression of the distribution, we plotted histograms of end-to-end RTT values as well as AS-end-RTTs of comparable AS measurements. Two sample histograms are shown in Fig. 7.1

The form of the histograms suggests that the RTT values follow a heavy-tailed distribution. According to [21], a distribution is heavy-tailed if its complementary cumulative distribution function (CCDF, cf. equation 7.2), often referred to as the tail, decays slower than exponentially, i.e., there is some $\gamma>0$ such that

$$
\lim _{t \rightarrow \infty} e^{\gamma t} F_{c}(t) \rightarrow \infty
$$

I.e., regardless of the behavior of the distribution for small values of the random variable, if the asymptotic shape of the distribution is hyperbolic, it is heavy-tailed [22]. The histograms we plotted clearly have an asymptotic hyperbolic shape as can be seen from the examples in Fig. 7.1 

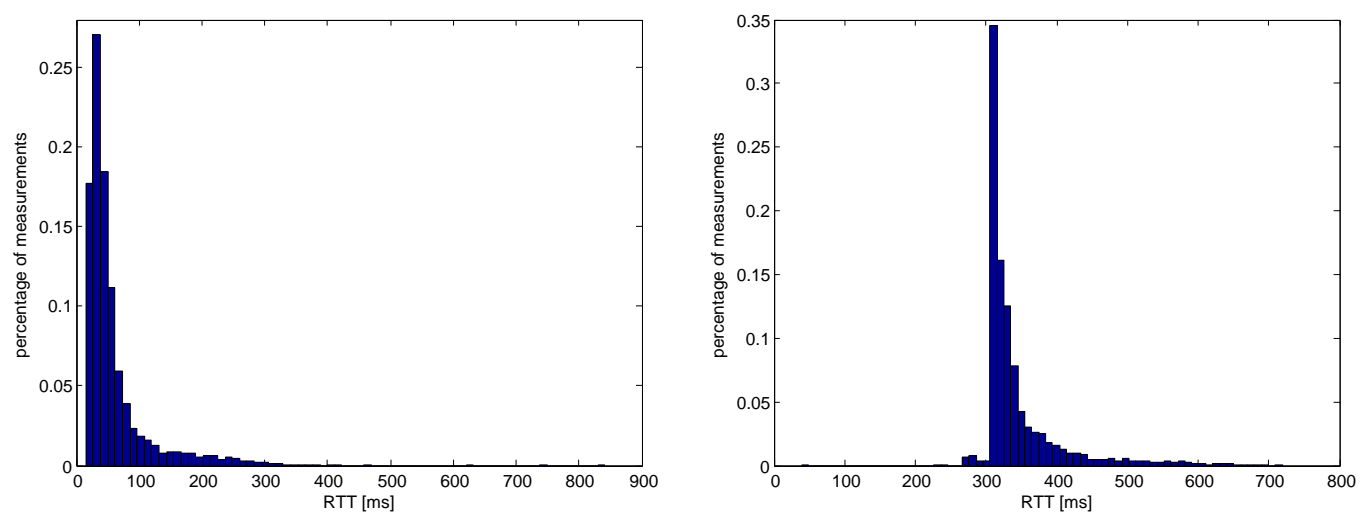

Figure 7.1: RTT value distribution of a sample subset of comparable AS-end-RTTs on the left. RTT value distribution of an end-to-end connection on the right.

A more accurate test to find out whether the distribution is heavy-tailed is by plotting the complementary cumulative distribution function (CCDF) on a log-log scale [23]. The CCDF is defined as:

$$
F_{c}(x)=P(X>x)=1-F(x)
$$

where $\mathrm{F}(\mathrm{x})$ is the cumulative distribution function (CDF). The CCDF represents the probability that the random variable $X$ takes on a value greater than or equal to $\mathrm{x}$.

If the data follows a heavy-tailed distribution, then the log-log plot of the CCDF results asymptotically linear. If the histogram is a straight line on log-log scales, then the following equation holds:

$$
\ln p(x)=-\alpha \ln x+c
$$

where $\alpha$ and c are constants. Taking the exponential of both sides, this is equivalent to:

$$
p(x)=C x^{-\alpha}
$$

with $C=e^{c}$. Distributions of the form 7.4 are said to follow a "power law" [23]. Power law distributions are a special kind of heavy-tailed distributions.

To get more certainty about whether our RTT measurement data really follows a heavy-tailed or even a power law distribution we plotted the CCDF on log-log scale. Sample plots are shown in Fig. 7.2

As can be seen from the sample plots, the measurements clearly follow a heavy-tailed distribution. Therefore the COV-which is defined as $c_{v}=\sigma / \mu$-is not an appropriate measure to express the stability, since heavy-tailed distribution mostly have infinite variance and often an infinite mean. Thus the interquartile range is the appropriate measure for RTT stability. 

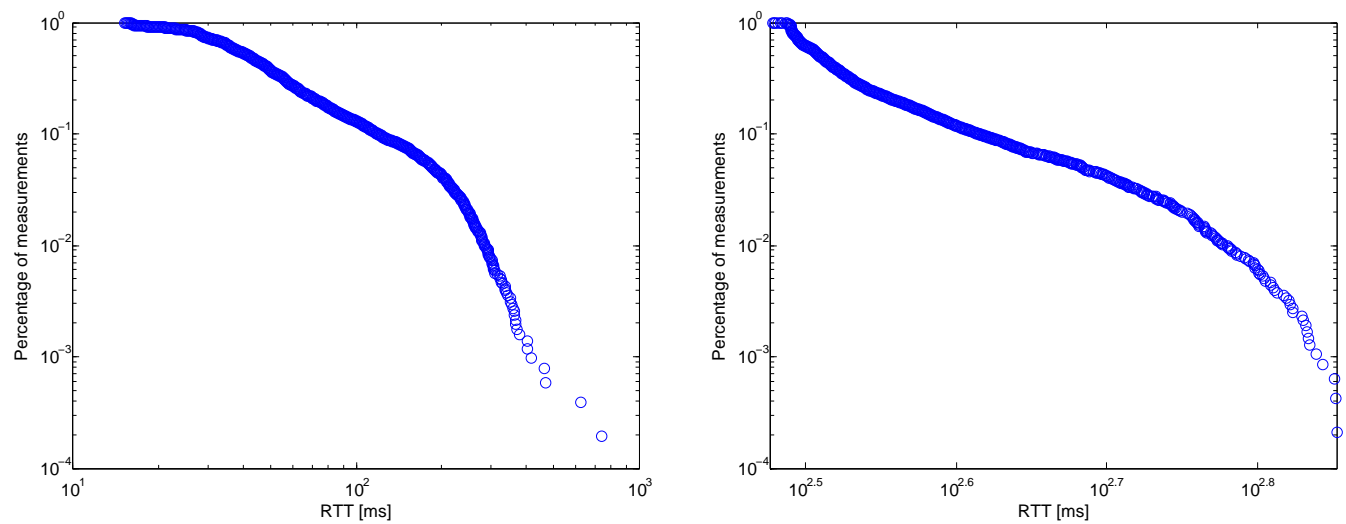

Figure 7.2: Log-log plot of the CCDF of a sample subset of comparable AS-end-RTTs on the left and of the RTT value distribution of an end-to-end connection on the right.

\subsubsection{Interquartile Range}

The interquartile range (IQR) is a measure of statistical dispersion, being equal to the difference between the third and first quartiles [24]. A quartile is any of the three values which divide the sorted data set into four equal parts, so that each part represents one fourth of the sampled population [25]. The IQR can be applied without making any assumptions of the underlying statistical distribution and is therefore particularly applicable for heavy-tailed distributions.

However, the IQR as such cannot be compared among AS's, since the range of their RTT values might vary significantly. We have to "normalize" the IQR somehow to derive a comparable value. One feasible possibility is to compute the ratio of the IQR to the median. The median can be-as well as the other quartiles - calculated without any assumption of the underlying distribution. The value for stability is now defined as

$$
s=\frac{I Q R}{\text { Median }}=\frac{Q_{3}-Q_{1}}{Q_{2}}
$$

where $Q_{i}$ denotes the i-th quartile. This value can be computed for all comparable AS-measurements of the same AS. The median of all those values can now be compared to the values of other AS's resulting in an actual quality measure.

\subsubsection{AS-RTT}

After specifying the delay stability measure, we applied it to the AS-RTT of some sample measurements. During this analysis we discovered the traceroute problem discussed in Sect. 4.5 which lead us to our new approach. 


\subsubsection{Traceroute Configurations}

Another initial test was the comparison of the traceroute configurations. As described in Sect. 5.1.2. we performed our measurements using the ICMP and TCP protocol and using minimal and maximal packet payloads.

To analyze the differences of the configurations we computed the median RTT of each configuration for every end-to-end connection, using the Perl script fetch_config_stats.pl. We ranked the configurations according to their median RTT for every end-to-end connection and added up the ranks.

The results show how often, i.e., for how many end-to-end connections, a configuration has the lowest, second lowest, third lowest, and highest median RTT. The output of the analysis shows that the configuration using TCP and an empty payload delivered the best results:

$\begin{array}{rrrr}\text { ICMP, MIN } & \text { ICMP, MTU } & \text { TCP, MIN } & \text { TCP, MTU } \\ \text { 1. } 68 & \text { 1. } 61 & \text { 1. } 113 & \text { 1. } 53 \\ \text { 2. } 92 & \text { 2. } 53 & \text { 2. } 70 & 2.80 \\ \text { 3. } 83 & \text { 3. } 63 & \text { 3. } 54 & \text { 3. } 95 \\ \text { 4. } 52 & \text { 4. } 118 & \text { 4. } 58 & 4.67\end{array}$

\subsection{Evaluating the Rating Approach}

To evaluate our rating approach we analyzed the data of our new database. The results of our analysis are described in the following sections. All SQL queries used to produce the results can be found in Appendix C.

\subsubsection{Overall Statistics}

The measurements we analyzed traversed 223 different Autonomous Systems. They were performed during 38 days between 22nd April and 29th May 2008.

Measurements which did not reach their destination were used to determine the connectivity-ratio as described in Sect. 4.6.2. Measurements which include one or more unknown AS-end-RTTs were considered as invalid and did not contribute to the quartile computations and to the rating at all. The other $63 \%$ of the performed measurements, namely $10^{\prime} 095^{\prime} 928$, were included in the rating. $4 \%$ of those measurements are outliers. For $57 \%$ of the outliers the guilty AS could be determined.

Total number of measurements:

$15^{\prime} 991^{\prime} 277$

Number of measurements that did not reach their destination: $\quad 1^{\prime} 588^{\prime} 900$

Number of valid measurements which contributed to the rating: $10^{\prime} 095^{\prime} 928$

Total number of outliers: $\quad 406^{\prime} 084$

Number of outliers for which the guilty AS could be determined: 234'137 


\begin{tabular}{c|c|c|c|c} 
Rank & ASN & \# Measurements & \# Outliers & Outlier-Ratio \\
\hline 1 & 16260 & 37691 & 0 & 0 \\
2 & 24748 & 12931 & 0 & 0 \\
3 & 513 & 7781 & 0 & 0 \\
4 & 12695 & 7321 & 0 & 0 \\
5 & 2607 & 6416 & 0 & 0 \\
6 & 8501 & 6369 & 0 & 0 \\
7 & 41398 & 6016 & 0 & 0 \\
8 & 4635 & 3546 & 0 & 0 \\
9 & 8434 & 3534 & 0 & 0 \\
10 & 39821 & 3198 & 0 & 0 \\
$\vdots$ & $\vdots$ & $\vdots$ & $\vdots$ & $\vdots$ \\
212 & 5432 & 301352 & 7696 & 0.026203341 \\
213 & 3300 & 96572 & 2527 & 0.026581812 \\
214 & 2609 & 99565 & 2767 & 0.028331541 \\
215 & 10429 & 96862 & 2805 & 0.028733277 \\
216 & 8447 & 100370 & 2813 & 0.028824084 \\
217 & 8289 & 100413 & 3218 & 0.033101207 \\
218 & 14551 & 100184 & 3538 & 0.036561295 \\
229 & 1836 & 100394 & 4868 & 0.050945015 \\
220 & 5384 & 100337 & 4997 & 0.05237122 \\
221 & 19262 & 100416 & 5368 & 0.056458314
\end{tabular}

Table 7.1: Top ten and worst ten intra-AS qualities

\subsubsection{Intra-AS Rating}

To determine the intra-AS quality of the AS's we computed the outlier-ratio as well as the connectivity-ratio of each AS as described in Sect. 4.6.2.

Examining the connectivity ratios, we realized that there are AS's which seem to filter traceroute measurements. A large amount of our measurement connections ended in certain AS's without reaching their destination. Examples are AS16371 and AS12301, which filtered over 99\%.

AS2828 even filtered each of the 21'396 measurements which tried to traverse it. These could be AS's whose routers do not send ICMP responses to packets whose TTL is expired.

Due to this fact we cannot assume that interrupted measurements imply no connectivity.

Therefore we exclude the connectivity-ratio from the intra-AS rating and only rely on the outlier-ratio. In other words, in our intra-AS quality definition (4.4), we set the weight $\beta=0$.

First we produced an intra-AS ranking of all rated AS. The ranking rates the AS's according to their outlier-ratio. The top ten Autonomous Systems of our 38-days rating as well as the worst ten are shown in Table 7.1. The complete ranking can be found in Appendix D.1.

The column in the middle of Table 7.1 shows how many times the AS was traversed by a valid measurement. Every measurement which reached its destination and contains all AS-end-RTTs is valid. The next column shows how often the AS caused an outlying end-to-end RTT. Finally the last column presents the outlier-ratio, the actual intra-AS quality measure.

As can be seen from the ranking, there are AS's which were much more often traversed than others. Therefore the statement about the rating is more reliable for certain AS's than for others. The more often an AS was traversed by measurements, the more reliable the statement that we can make about its quality. 


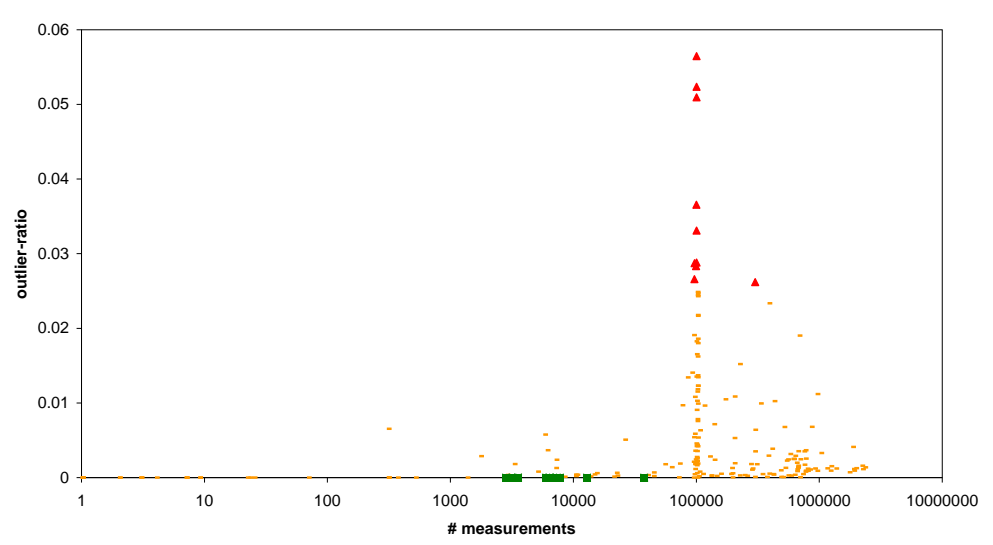

Figure 7.3: Scatter plot of the intra-AS qualities

\begin{tabular}{c|c|c|c|c|c} 
ASN & neighbor ASN & count & total count & weight & neighbor quality \\
\hline 1120 & 5588 & 1644 & 3343 & 0.491773856 & 0.001656571 \\
1120 & 8447 & 1654 & 3343 & 0.494765181 & 0.028824084 \\
1120 & 8514 & 2 & 3343 & 0.000598265 & 0.012304251 \\
1120 & 12558 & 43 & 3343 & 0.012862698 & 0.005413245
\end{tabular}

Table 7.2: Neighbors of AS1120

To express not only the quality but also the reliability of the rating, we produced a scatter plot of the results. A scatter plot is a type of display using Cartesian coordinates to display values for two variables for a set of data [29]. We plotted the number of measurements an AS was involved versus its outlier-ratio (refer to Appendix E to see different variants of the scatter plot and their pros and cons). Figure 7.3 shows the scatter plot of the complete intra-AS rating. The $x$-axis is displayed on a logarithmic scale. The top ten AS are displayed as green squares whereas the worst ten are the red triangles.

The scatter plot can be interpreted as follows: The lower an AS on the plot, the better its outlier-ratio, i.e., the better its intra-AS quality. The more an AS to the right of the plot, the more reliable the statement about its quality. Given an AS whose quality we want to examine we can highlight it in the plot an easily compare it to the other AS's. We can compare it to the quality of the others by comparing the vertical position and we can see how reliable the quality statement of the AS is by checking its horizontal position. Section 7.2.4 illustrates how the scatter plot can be used to compare the quality of AS's.

\subsubsection{Inter-AS Rating}

To make the inter-AS rating we queried for each AS the neighbors to which it routed connections during the measurements. We counted how often connections were routed to any neighbor, and how often the AS forwarded connections to each neighbor. Table 7.2 shows the results of an example AS.

The inter-AS quality is computed by the following formula presented in Sect. 4.6 .2 


\begin{tabular}{c|c|c|c} 
Rank & ASN & Routing Count & Inter-AS Quality \\
\hline 1 & 8501 & 3264 & 0 \\
2 & 13284 & 8 & 0 \\
3 & 41398 & 3264 & 0 \\
4 & 3786 & 314902 & 0.0000884441 \\
5 & 9831 & 361377 & 0.000217192 \\
6 & 513 & 3541 & 0.000283712 \\
7 & 6667 & 1475 & 0.000439267 \\
8 & 15857 & 217284 & 0.000525648 \\
9 & 34209 & 3215 & 0.000527884 \\
10 & 13046 & 305660 & 0.00056591 \\
$\vdots$ & $\vdots$ & $\vdots$ & $\vdots$ \\
148 & 6746 & 232503 & 0.023352534 \\
149 & 4657 & 5972 & 0.024281543 \\
150 & 7568 & 73017 & 0.02428551 \\
151 & 5588 & 83847 & 0.024444512 \\
152 & 6739 & 1 & 0.024735451 \\
153 & 8422 & 74749 & 0.024869873 \\
154 & 5400 & 60204 & 0.025146409 \\
155 & 6774 & 88327 & 0.026203341 \\
156 & 27699 & 52881 & 0.028733277 \\
157 & 8961 & 74139 & 0.05237122
\end{tabular}

Table 7.3: Top ten and worst ten inter-AS qualities

$$
\begin{array}{r}
\text { inter_AS_quality }\left(A S_{x}\right)=\sum_{A S_{i} \in N} \text { weight }\left(A S_{i}\right) * \text { intra_AS_quality }\left(A S_{i}\right) \\
\text { where } N=\left\{\text { all neighbors of } A S_{x}\right\}
\end{array}
$$

The weight of each neighbor is the ratio between the number of times the AS routed a connection to the neighbor and the number of times the AS routed a connection to any neighbor (columns "count" and "total count" in the table). Then we multiply the weight of each neighbor with its intra-AS quality (column "neighbor quality"). The sum of all weighted neighbor qualities results in the inter-AS quality of the AS (cf. Sect. 4.6.2). In the above example, the inter-AS quality of AS1120 is therefore:

$$
\begin{aligned}
& \text { inter_AS_quality }(A S 1120)=0.491773856 * 0.001656571+0.494765181 * 0.028824084+ \\
& 0.000598265 * 0.012304251+0.012862698 * 0.005413245=0.015152802
\end{aligned}
$$

Again we created a ranking as well as a scatter plot. The inter-AS top ten Autonomous Systems of our 38-days rating as well as the worst ten are shown in the Table 7.3. The complete ranking can be found in Appendix D.2 


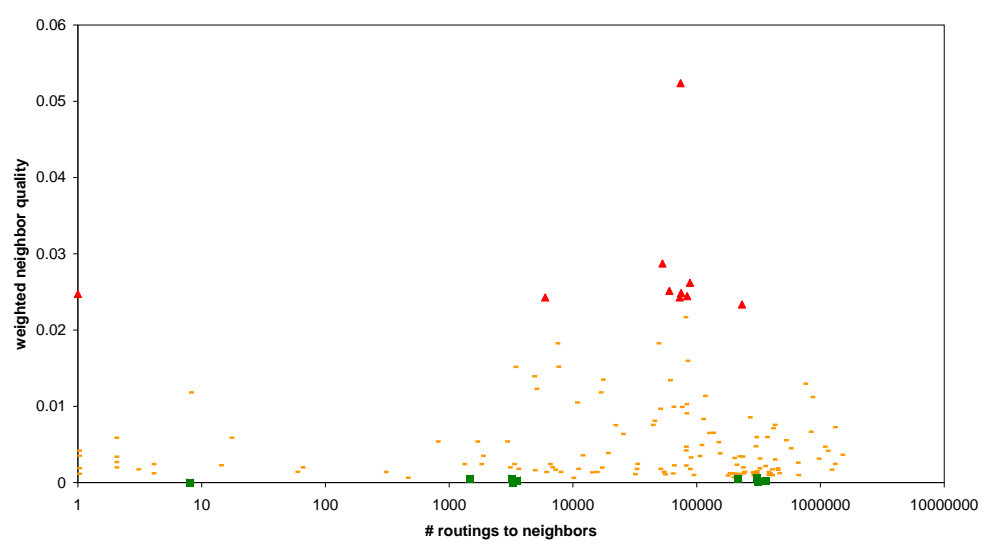

Figure 7.4: Scatter plot of the inter-AS quality rating

The scatter plot in Fig. 7.4 plots the number of times an AS routed a connection to another AS against the weighted sum of the intra-AS qualities of all its neighbors. The lower an AS on the plot the better the qualities of its neighbors. The more the AS to the right of the plot the more reliable the statement about its inter-AS quality.

Given an AS whose quality we want to examine we can highlight it in the plot and easily compare it to the other AS's. We can compare it to the inter-AS quality of the others by comparing the vertical position and we can see how reliable the quality statement of the AS is by checking its horizontal position. Section 7.2.4 illustrates how the scatter plot can be used to compare the quality of AS's.

\subsubsection{AS Rating for ISP Selection}

The following example shows, how our AS rating can be used to support the selection of an ISP: Two of our measurement points are located in South Korea. They are connected to two different ISPs, i.e., to two different AS's. One site is connected to the ISP "LG Dacom" (AS3786), the other one to "Korea Telecom" (AS4766).

\section{Overall}

To find out whether one of the AS's performs better than the other we first analyze their place in the rankings. The rankings provide the following results for the two AS's:

$\begin{array}{lll}\text { ASN } & \text { Intra-AS Quality } & \text { Inter-AS Quality } \\ 3786 & 148 / 221 & 4 / 157 \\ 4766 & 203 / 221 & 61 / 157\end{array}$

From the ranking we can see, that AS3786 is of better intra-AS quality as well as better inter-AS quality than AS4766. Figure 7.5 highlights the two AS's in the scatter plots of the intra-AS quality (on top) and inter-AS quality rating (at the bottom). 

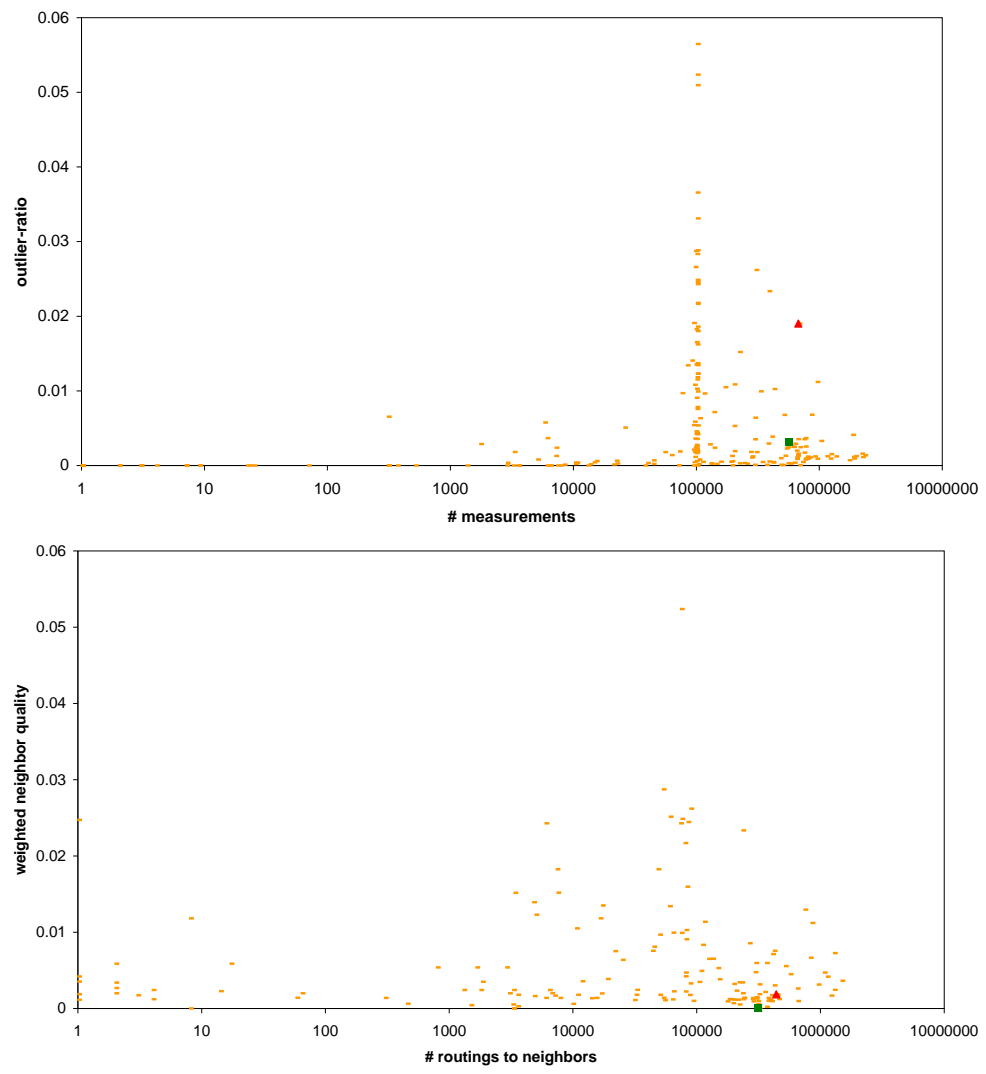

Figure 7.5: AS3786 is represented by the green square whereas AS4766 is shown as red triangle. Intra-AS quality is shown on top, inter-AS quality at the Bottom 


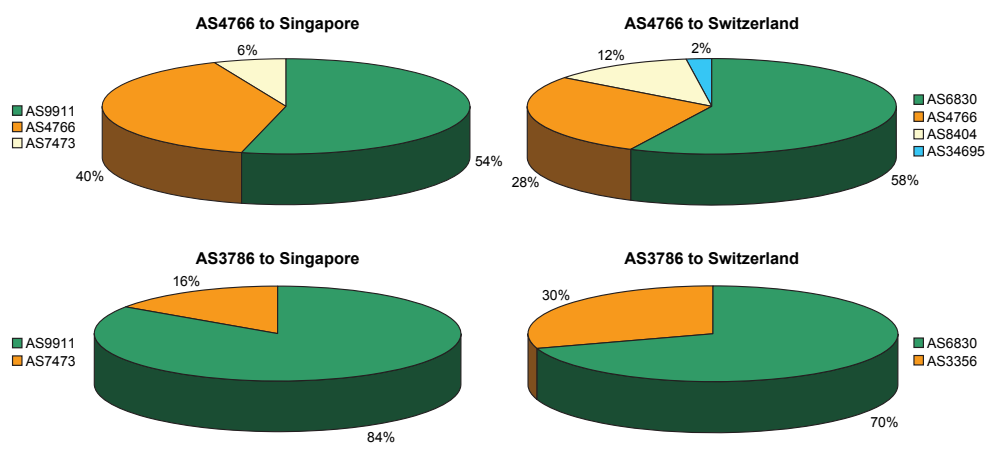

Figure 7.6: End-to-end outliers

For both qualities we can see, that the reliability of the statements about the two AS's are similar and therefore a comparison is justified.

\section{End-to-End Connections}

In our context, the measurement points are VPN sites. The important connections of the VPN environment are the connections to other VPN sites. For the South Korean sites the most important connections are connections to sites in Singapore and in Switzerland.

In order to chose the best ISP for the South Korean sites, we further analyze the measurements of the end-to-end connections between the South Korean sites and the destinations in Singapore and in Switzerland.

Figure 7.6 shows that AS3786 did not cause any outlier on the connections to Switzerland and Singapore. AS4766, however, caused $28 \%$ and $40 \%$ of the outliers observed on these connections.

\section{Conclusion}

All our tests resulted in AS3786 being a better AS than AS4766. Therefore we suggest to prefer "LG Dacom" to "Korea Telecom" as Internet Service Provider in South Korea.

\subsubsection{AS Rating for Real-Time Debugging}

For real-time debugging support, the AS rating can be used as follows: If performance problems on an end-to-end connection are experienced, a traceroute measurement is performed on that connection. From the traceroute output the AS-route, the end-to-end RTT, and all AS-end-RTTs are determined. By comparing the end-to-end RTT to the respective quartiles in the database it is determined whether the RTT of the measurement is outlying. If yes, the outlier test of all AS's on the AS-route is performed as well to find out which AS causes the connection problems.

Assume this traceroute output taken from our measurements: 


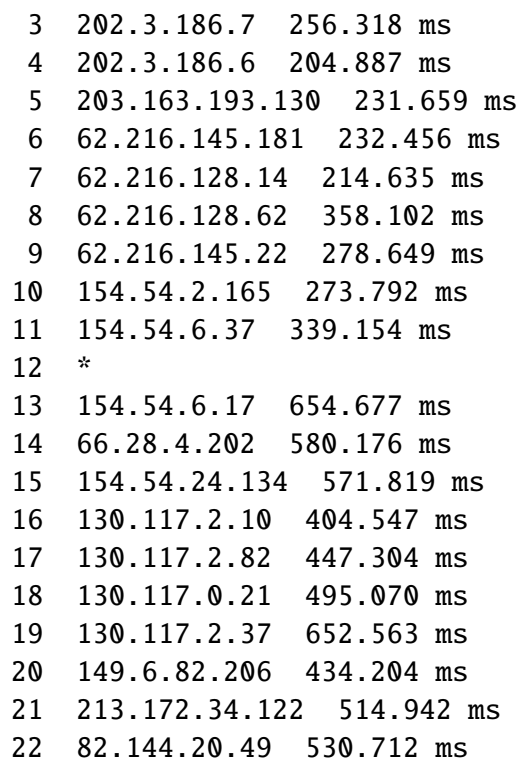

Table 7.4 shows the AS to which each host on the route belongs. Furthermore it lists the quartiles of each AS-end-RTT (columns "Q1" and "Q3") and whether the corresponding time is outlying (column "Outlier").

The shown measurement traverses the AS's 9831, 15412, 174, and 16206 in the given order. As can be seen from Table 7.4. AS174 is the first outlier on the AS-route. Since the following AS on

\begin{tabular}{|c|c|c|c|c|c|c|c|}
\hline & IP & ASN & RTT [ms] & Q1 [ms] & Q3 [ms] & Threshold [ms] & Outlier \\
\hline 1 & 202.3.175.185 & 9831 & 40.480 & & & & \\
\hline 2 & 202.3.190.69 & 9831 & 160.828 & & & & \\
\hline 3 & 202.3.186.7 & 9831 & 256.318 & & & & \\
\hline 4 & 202.3.186.6 & 9831 & 204.887 & & & & \\
\hline 5 & 203.163.193.130 & 9831 & 231.659 & 76.592 & 170.823 & 453.516 & no \\
\hline 6 & 62.216 .145 .181 & 15412 & 232.456 & & & & \\
\hline 7 & 62.216 .128 .14 & 15412 & 214.635 & & & & \\
\hline 8 & 62.216 .128 .62 & 15412 & 358.102 & & & & \\
\hline 9 & 62.216.145.22 & 15412 & 278.649 & 179.284 & 247.499 & 452.144 & no \\
\hline 10 & 154.54.2.165 & 174 & 273.792 & & & & \\
\hline 11 & 154.54.6.37 & 174 & 339.154 & & & & \\
\hline 12 & * & & & & & & \\
\hline 13 & 154.54.6.17 & 174 & 654.677 & & & & \\
\hline 14 & 66.28 .4 .202 & 174 & 580.176 & & & & \\
\hline 15 & 154.54.24.134 & 174 & 571.819 & & & & \\
\hline 16 & 130.117.2.10 & 174 & 404.547 & & & & \\
\hline 17 & 130.117.2.82 & 174 & 447.304 & & & & \\
\hline 18 & 130.117.0.21 & 174 & 495.070 & & & & \\
\hline 19 & 130.117.2.37 & 174 & 652.563 & & & & \\
\hline 20 & 149.6.82.206 & 174 & 434.204 & 314.837 & 335.816 & 398.753 & yes \\
\hline 21 & 213.172 .34 .122 & 16206 & 514.942 & & & & \\
\hline 22 & 82.144 .20 .49 & 16206 & 530.712 & 316.585 & 333.149 & 382.841 & yes \\
\hline
\end{tabular}

Table 7.4: Real-time debugging 
the route-AS 16206- has an outlying AS-end-RTT as well, AS174 can be blamed to be causing the bad performance.

This example shows how our approach can be applied in practice for real-time Internet debugging. In the given scenario we spotted the weak point of the poor Internet connection. In the case of ongoing connection problems the operator of the guilty AS can be informed and urged to solve the issue. 


\section{Chapter 8}

\section{Conclusions}

\subsection{Summary}

In this master's thesis we approached the following research question:

How can Autonomous Systems automatically, efficiently, and in a meaningful and reliable way be rated by their quality in real-time as well as over long periods of time?

We developed an approach to rate Autonomous Systems according to their network quality (referred to as intra-AS quality) as well as their peering quality (referred to as inter-AS quality). The network quality is based on how often an AS causes an outlying end-to-end round-trip time on a connection passing through it. An outlier is defined as exceeding the median RTT by more than three interquartile ranges. This definition copes with the noisy measurement data and the heavy-tailed distribution of the RTT measurements. The less outliers an AS causes the better its network quality.

To determine its peering quality we sum up the weighted network qualities of the neighboring AS's of an AS. The weight of a neighbor is determined by the frequency the AS routes packets to it.

To test our approach we developed a prototype framework. We performed 16 millions measurements during 38 days and analyzed the gathered data using scatter plots and quality rankings of all tested AS's.

\subsection{Conclusions}

The major challenge of our work was the collection of reliable network measurement data. While it is easily possible to make accurate end-to-end RTT measurements, it turned out to be very difficult to make accurate and reliable measurements of the intermediate parts of an Internet connection. Our measurements had to be done without access to the network infrastructure, namely the routers and switches between source and destination. Therefore we relied on traceroute measurements. However, traceroute measurements can be biased by slowly answering routers. 
Taking the measurement difficulties into account, we developed an outlier-based approach to rate Autonomous Systems. We created an ordered ranking of the long-term qualities of all evaluated AS's. Furthermore, our outlier detection method can be used to detect the AS causing a slow Internet connection in real-time.

Unfortunately we can not be sure, whether our definition of AS quality really represents the true overall quality of an Autonomous System.

\subsection{Future Work}

\subsubsection{Traceroute}

During our work we experienced problems with traceroute measurements which made it difficult for us to ensure the reliability of our rating. The most important future work is the thorough analysis of traceroute measurements to enable a statement about the reliability of our rating approach.

\subsubsection{Enhancing our Rating Approach}

In our rating approach, end-to-end outliers for which no guilty AS can be determined are not further analyzed. Those outliers occur if there are certain AS-routes which are significantly slower than other AS-routes on the same end-to-end connection. Future work could define a way to include these outliers in the rating.

\subsubsection{Alternative Rating Approach}

The outlier-approach we used to derive the rating from the measurement data is one possible approach. Future work could try other approaches and compare the results to our rating. One possible alternative approach is indicated in Sect. 4.6.1.

\subsubsection{Implementation for use in Practice}

Once we are aware of the reliability of the traceroute results, the prototype framework can be adapted to a framework applicable in practice.

\subsubsection{Long-Term Evaluation}

Future work could apply our rating approach over an extended period of time-at least one year-to determine the consistency of the rating. Furthermore the rating could be compared to different kinds of measurements. These tasks would verify our rating approach on how well it represents the true quality of an AS. 


\subsection{Application in Practice}

In order to apply our AS rating in practice, several parts of our prototype framework must be adapted.

Data Preprocessing To keep the computation time on the central data repository as low as possible, the measurement points preprocess the measurement data as much as possible. They summarize each measurement to the following information:

- source

- destination

- end-RTT

- AS-route

- AS-end-RTTs

This set of information suffices to update the central database without further preprocessing.

Data Delivery To have a continuous up-to-date ranking, measurement results must be delivered immediately to the central database. After each measurement-and its preprocessing - data is immediately sent to the central data repository in order to update the database.

Time Window To reflect the current qualities, the rating of Autonomous Systems is based on measurement data of a specific period of time, i.e., $n$ weeks. Measurements which are older than $n$ weeks do not influence the rating. Since the measurement values are not preserved, but the quartiles of the RTTs only updated, it is not possible to exclude former measurements from the database. Therefore the content of a database that contains measurements older than $n$ weeks has to be discarded and replaced by new content. However, in order not to start from scratch every $n$ weeks, multiple databases are kept at the same time. Every week a new database is started to be filled. After $n$ weeks the oldest one is discarded while the next one-containing data of $n-1$ weeks-is taken over. Hence the rating is always based on data of at least $n-1$ weeks, but never on data which is older than $n$ weeks.

Outlier Determination Whenever an end-to-end outlier is observed, multiple additional measurements are performed. This assures that an observed end-to-end outlier is not only a temporary peak, but a real connection problem. Furthermore it makes the determination of the guilty AS more reliable for the same reason. 


\section{Appendix A}

\section{Database Fields}

\section{A.1 Initial Database Scheme}

Every table contains a field "id" which holds a unique integer value identifying each record. The id field is not included in the explanations below.

\section{A.1.1 Table "autonomous_system"}

Every traceroute measurement traverses multiple AS's. The fields of the table holding the corresponding information of each traversed AS have the following meaning:

\begin{tabular}{l|l} 
Field name & Description \\
\hline asn & AS number of the traversed AS \\
\hline dist_int & Geographical length of the path within the AS \\
\hline dist_out & Geographical length from the last host within the AS to the destination \\
\hline from_host & $\begin{array}{l}\text { Host which is immediately before the first host of this AS on the measure- } \\
\text { ment path (foreign key on field "id" of table "host") }\end{array}$ \\
\hline ip_count & Number of hosts within the AS \\
\hline loss_int & Number of lost packets within the AS \\
\hline loss_border & Number of lost packets on the connections to the previous and next AS \\
\hline measurement & $\begin{array}{l}\text { Measurement to which the traversed AS belongs (foreign key on field "id" } \\
\text { of table "measurement") }\end{array}$ \\
\hline rtt & AS-RTT in the corresponding measurement \\
\hline seq & Position of the AS among all AS's of the measurement \\
\hline to_host & $\begin{array}{l}\text { Host immediately after the AS in the corresponding measurement (foreign } \\
\text { key on field "id" of table "host") }\end{array}$
\end{tabular}




\section{A.1.2 Table "config"}

Each measurement is done with a certain traceroute configuration. The configuration attributes are the following:

\begin{tabular}{l|l} 
Field name & Description \\
\hline protocol & Network protocol used for the measurement \\
\hline size & Payload size of the probes
\end{tabular}

\section{A.1.3 Table "end_host"}

Each measurement is done from a certain source host to a certain destination host. Sources and destinations are stored in a table containing the following fields:

\begin{tabular}{l|l} 
Field name & Description \\
\hline asn & AS number of the AS to which the end host belongs \\
\hline hostname & Hostname of the end host \\
\hline ip & IP address of the end host
\end{tabular}

\section{A.1.4 Table "host"}

Every time a host is traversed by a measurement it is stored in the host table. The fields of the host table have the following meanings:

\begin{tabular}{l|l} 
Field name & Description \\
\hline asn & AS number of the AS to which the host belongs \\
\hline ip & IP address of the host \\
\hline latitude & Latitude of the host \\
\hline longitude & Longitude of the host \\
\hline measurement & $\begin{array}{l}\text { Measurement to which the host belongs (foreign key on field "id" of table } \\
\text { "measurement") }\end{array}$ \\
\hline rtt & RTT to the host in the corresponding measurement \\
\hline seq & Position of the host among all hosts of the measurement
\end{tabular}

\section{A.1.5 Table "measurement"}

The relevant information of each measurement is stored in a table with the following fields: 


\begin{tabular}{l|l} 
Field name & Description \\
\hline config & $\begin{array}{l}\text { Configuration of the measurement (foreign key on field "id" of table "con- } \\
\text { fig") }\end{array}$ \\
\hline dest & $\begin{array}{l}\text { Destination host of the measurement (foreign key on field "id" of table } \\
\text { "end_host") }\end{array}$ \\
\hline $\mathrm{rtt}$ & End-to-end RTT \\
\hline src & $\begin{array}{l}\text { Source host of the measurement (foreign key on field "id" of table } \\
\text { "end_host") }\end{array}$ \\
\hline time & Time when the measurement was performed
\end{tabular}

\section{A.2 New Database Scheme}

The new database scheme is specific to the new approach. It includes much less information than the initial scheme.

Again every table contains a field "id" which holds a unique integer value identifying each record and which is not included in the explanations below. Furthermore the tables "as_route" and " $22 \mathrm{e}$ " contain multiple helper fields that are necessary for the quartile computation algorithm but are not mentioned below.

\section{A.2.1 Table "as_route"}

To be able to detect outlier RTTs of Autonomous Systems on a certain AS-route, the quartiles of each AS-end-RTT are stored in this table.

\begin{tabular}{l|l} 
Field name & Description \\
\hline as_path & $\begin{array}{l}\text { AS numbers of all AS's on the route (stored in the form } \\
\text { "ASN1:ASN2:...:ASNn") }\end{array}$ \\
\hline cnt & $\begin{array}{l}\text { Number of times this AS-route occurred on its corresponding end-to-end } \\
\text { measurement }\end{array}$ \\
\hline e2e & $\begin{array}{l}\text { End-to-end connection to which this AS-route belongs (foreign key on field } \\
\text { id" of table "e2e") }\end{array}$ \\
\hline rtt_q1s & $\begin{array}{l}\text { First quartiles of the AS-end-RTTs of all AS's on the AS route (stored in the } \\
\text { form "Qr1:Qr2:...:Qrn") }\end{array}$ \\
\hline rtt_q3s & $\begin{array}{l}\text { Third quartiles of the AS-end-RTTs of all AS's on the AS route (stored in the } \\
\text { form "Qr1:Qr2:...:Qrn") }\end{array}$
\end{tabular}

\section{A.2.2 Table "e2e"}

To detect end-to-end RTT outliers the quartiles of each end-to-end connection are stored among some other information about the connections. 


\begin{tabular}{l|l} 
Field name & Description \\
\hline dest & IP address of the destination of the connection \\
\hline rtt_q1 & First quartile of the RTT of the end-to-end connection \\
\hline rtt_q3 & Third quartile of the RTT of the connection \\
\hline src & Hostname of the source of the connection
\end{tabular}

\section{A.2.3 Table "measurement"}

To allow the analysis of the measurement data, a record for each measurement is held in this table.

\begin{tabular}{l|l} 
Field name & Description \\
\hline as_path & $\begin{array}{l}\text { AS numbers of all AS's on the route of this measurement (stored in the form } \\
\text { "ASN1:ASN2:...:ASNn") }\end{array}$ \\
\hline $\mathrm{e} 2 \mathrm{e}$ & $\begin{array}{l}\text { End-to-end connection on which the measurement was preformed (foreign } \\
\text { key on field "id" of table "e2e") }\end{array}$ \\
\hline $\mathrm{rtt}$ & RTT of the complete measurement \\
\hline $\mathrm{rtts}$ & AS-end-RTTs of all AS's on the AS-route of this measurement
\end{tabular}

\section{A.3 Extension of the new Database}

\section{A.3.1 Table "outlier_as"}

All outliers caused by a bad performing AS are stored in this table.

\begin{tabular}{l|l} 
Field name & Description \\
\hline as_route & $\begin{array}{l}\text { AS-route on which the outlier was observed (foreign key on field "id" of } \\
\text { table "as_route" }\end{array}$ \\
\hline asn & AS number of the AS which caused the outlier \\
\hline cnt & Number of times this outlier occured
\end{tabular}

\section{A.3.2 Table "outlier_unknown"}

Outliers for which no guilty AS can be determined are stored in this table.

\begin{tabular}{l|l} 
Field name & Description \\
\hline as_path & $\begin{array}{l}\text { AS numbers of all AS's on the route of this measurement (stored in the form } \\
\text { "ASN1:ASN2:...:ASNn") }\end{array}$ \\
\hline cnt & Number of times this outlier occured \\
\hline $\mathrm{e} 2 \mathrm{e}$ & $\begin{array}{l}\text { End-to-end connection on which this outlier occured (foreign key on field } \\
\text { "id" of table "e2e") }\end{array}$
\end{tabular}




\section{A.3.3 Table "unreached"}

Whenever the destination was not reached in a measurement it is recorded in this table.

\begin{tabular}{l|l} 
Field name & Description \\
\hline as_path & $\begin{array}{l}\text { AS numbers of all AS's on the route of this measurement until no response } \\
\text { got back (stored in the form "ASN1:ASN2:...:ASNn") }\end{array}$ \\
\hline $\mathrm{e} 2 \mathrm{e}$ & $\begin{array}{l}\text { End-to-end connection on which the measurement was preformed (foreign } \\
\text { key on field "id" of table "e2e") }\end{array}$ \\
\hline cnt & $\begin{array}{l}\text { Number of times this combination of end-to-end connection and AS-route } \\
\text { did not reach its destination }\end{array}$
\end{tabular}




\section{Appendix B}

\section{Software Versions}

The following table shows the exact versions of the software used throughout the thesis.

\begin{tabular}{l|l} 
Software & Version \\
\hline Matlab & $7.6 .0 .324(\mathrm{R} 2008 \mathrm{a})$ \\
\hline Microsoft Excel & $2003(11.8211 .8202) \mathrm{SP3}$ \\
\hline Paris-Traceroute & $0.92-\mathrm{dev}$ \\
\hline Perl & 5.8 .8 \\
\hline pgAdmin III & 1.8 .2 \\
\hline PostgreSQL & 8.3 .3 \\
\hline TCP-Traceroute & 1.5 beta7 \\
\hline Ubuntu & 8.04 with kernel 2.6.24-19-generic \\
\hline Whois & 4.7 .24
\end{tabular}




\section{Appendix C}

\section{Data Evaluation Queries}

\section{C.1 Overall Statistics}

This query produces the overall statistics of the measurements we analyzed:

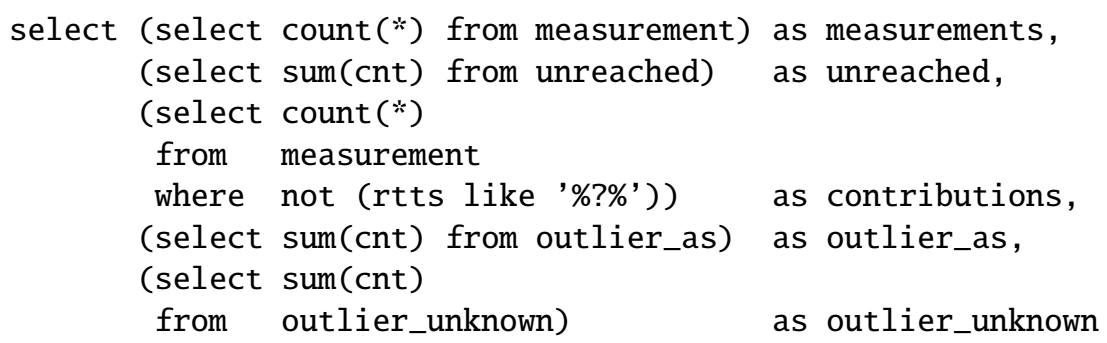




\section{C.2 Intra-AS Quality}

This query produces the complete ordered intra-AS ranking as shown in Appendix D.1.

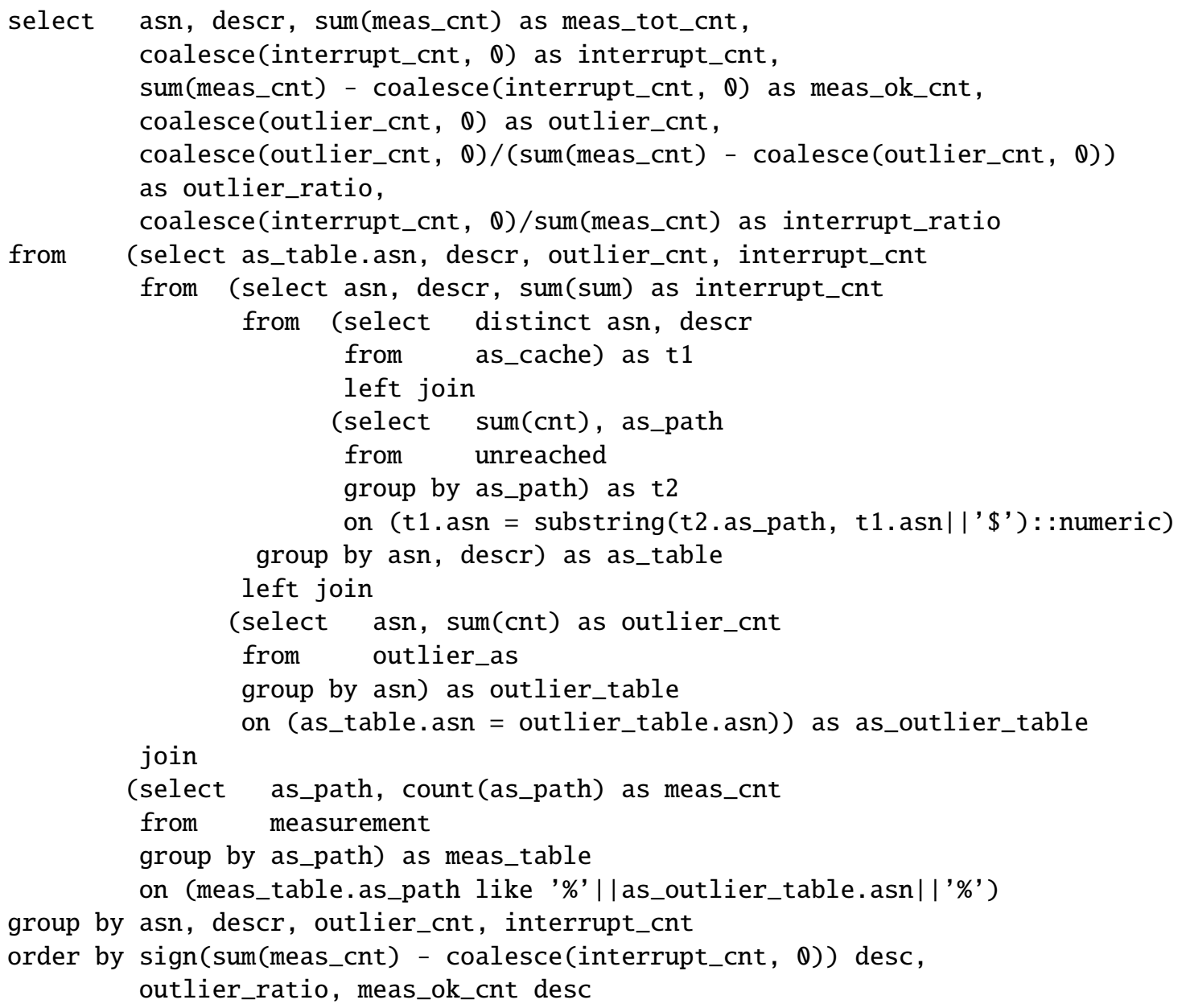




\section{C.3 Inter-AS Quality}

The following query fetches all neighbors of an AS as well as their weight according to the total number of routings. The actual rating (creating the weighted sum) was done with the help of Excel.

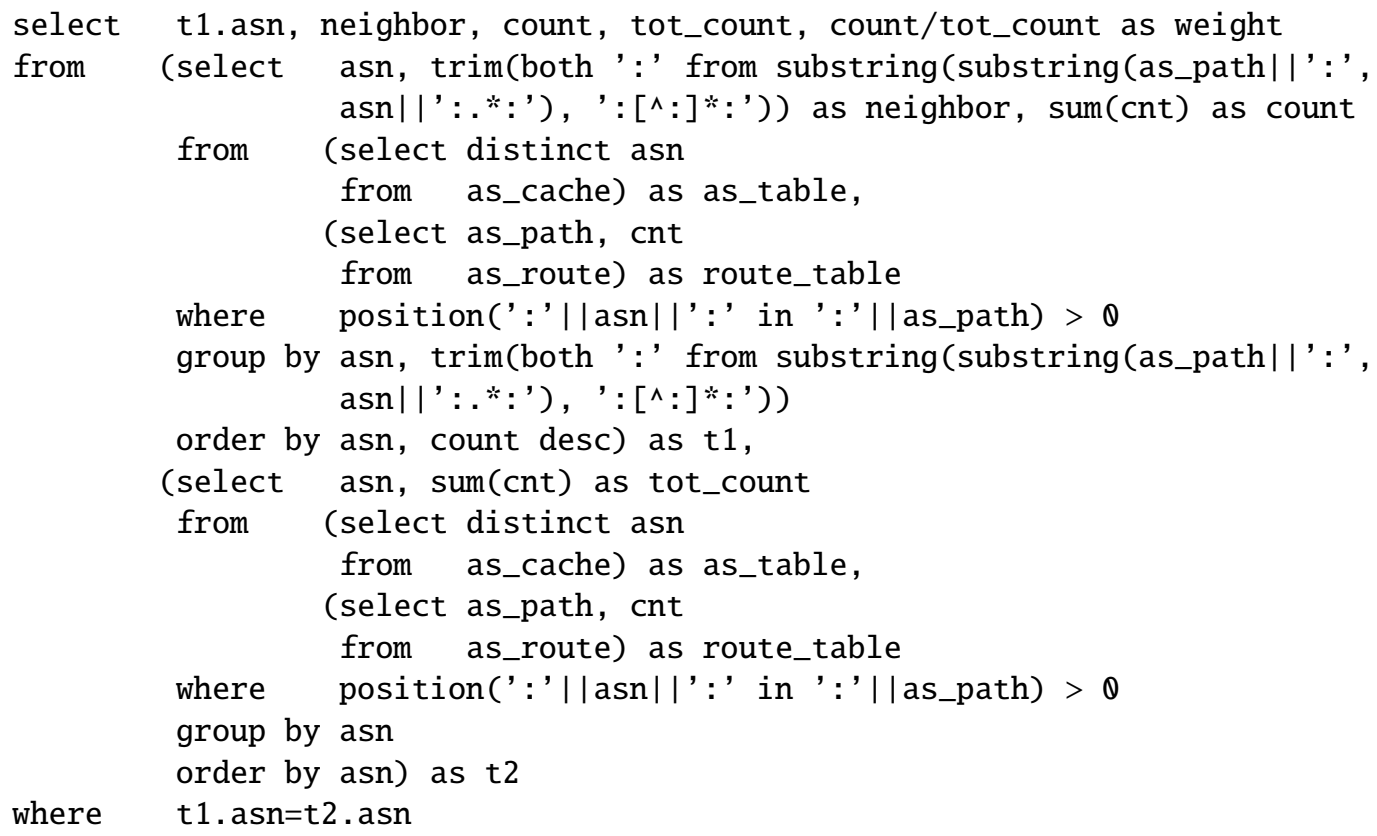




\section{C.4 AS End-to-End Rating}

This query determines for each end-to-end connection which AS caused how many outliers. Its results were used for the ISP comparison:

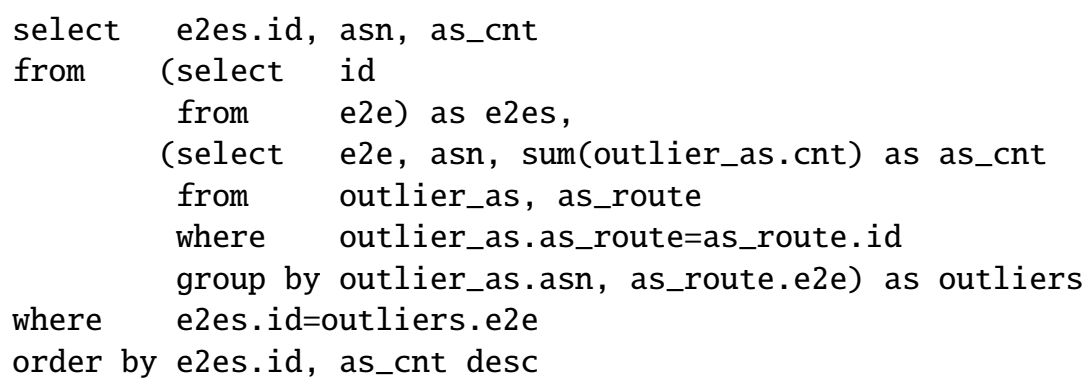




\section{Appendix D}

\section{AS Rankings}

\section{D.1 Intra-AS Quality}

This table shows the complete ranking of the intra-AS qualities of all Autonomous Systems which were traversed by our measurements. Column "\# meas total" shows the total number of measurements which included the AS; "\# interrupts" contains the number of times a measurement did not reach its destination and ended in the corresponding AS. Column "\# meas $\mathrm{ok}^{\prime \prime}$ is the number of valid measurements which included the AS, and the last columns contain the number of outliers an AS caused, and the outlier-ratio of the AS.

\begin{tabular}{|c|c|c|c|c|c|c|}
\hline Rank & ASN & \# meas total & \# interrupts & \# meas ok & \# outliers & outlier ratio \\
\hline 1 & 16260 & 37712 & 21 & 37691 & 0 & 0 \\
\hline 2 & 24748 & 12931 & 0 & 12931 & 0 & 0 \\
\hline 3 & 513 & 7781 & 0 & 7781 & 0 & 0 \\
\hline 4 & 12695 & 92875 & 85554 & 7321 & 0 & 0 \\
\hline 5 & 2607 & 6416 & 0 & 6416 & 0 & 0 \\
\hline 6 & 8501 & 6369 & 0 & 6369 & 0 & 0 \\
\hline 7 & 41398 & 6016 & 0 & 6016 & 0 & 0 \\
\hline 8 & 4635 & 3546 & 0 & 3546 & 0 & 0 \\
\hline 9 & 8434 & 3534 & 0 & 3534 & 0 & 0 \\
\hline 10 & 39821 & 3199 & 1 & 3198 & 0 & 0 \\
\hline 11 & 8342 & 2843 & 6 & 2837 & 0 & 0 \\
\hline 12 & 27908 & 1352 & 3 & 1349 & 0 & 0 \\
\hline 13 & 12555 & 512 & 0 & 512 & 0 & 0 \\
\hline 14 & 30721 & 366 & 0 & 366 & 0 & 0 \\
\hline 15 & 21060 & 308 & 0 & 308 & 0 & 0 \\
\hline 16 & 16371 & 92658 & 92350 & 308 & 0 & 0 \\
\hline 17 & 12714 & 69 & 0 & 69 & 0 & 0 \\
\hline 18 & 19422 & 25 & 0 & 25 & 0 & 0 \\
\hline 19 & 29259 & 23 & 0 & 23 & 0 & 0 \\
\hline 20 & 22773 & 22 & 0 & 22 & 0 & 0 \\
\hline 21 & 9026 & 9 & 0 & 9 & 0 & 0 \\
\hline 22 & 13284 & 9 & 0 & 9 & 0 & 0 \\
\hline 23 & 4755 & 10 & 3 & 7 & 0 & 0 \\
\hline
\end{tabular}




$\begin{array}{lllllll}24 & 22351 & 50 & 43 & 7 & 0 & 0 \\ 25 & 30126 & 7 & 0 & 7 & 0 & 0 \\ 26 & 6731 & 4 & 0 & 4 & 0 & 0 \\ 27 & 8696 & 3 & 0 & 3 & 0 & 0 \\ 28 & 8767 & 3 & 0 & 3 & 0 & 0 \\ 29 & 15972 & 16 & 13 & 3 & 0 & 0 \\ 30 & 29076 & 2 & 0 & 2 & 0 & 0 \\ 31 & 9105 & 4 & 2 & 2 & 0 & 0 \\ 32 & 4713 & 1 & 0 & 1 & 0 & 0 \\ 33 & 2854 & 1 & 0 & 1 & 0 & 0 \\ 34 & 2874 & 1 & 0 & 1 & 0 & 0 \\ 35 & 8764 & 2 & 1 & 1 & 0 & 0 \\ 36 & 13237 & 1 & 0 & 1 & 0 & 0 \\ 37 & 12322 & 1 & 0 & 1 & 0 & 0 \\ 38 & 6739 & 1 & 0 & 1 & 0 & 0 \\ 39 & 39010 & 99771 & 28 & 99743 & 1 & 0.000010023 \\ 40 & 8402 & 96737 & 3733 & 93004 & 1 & 0.000010337 \\ 41 & 23520 & 70816 & 255 & 70561 & 1 & 0.000014121 \\ 42 & 12301 & 99835 & 99526 & 309 & 2 & 0.000020033 \\ 43 & 18118 & 196099 & 3836 & 192263 & 5 & 0.000025498 \\ 44 & 209 & 681351 & 49 & 681302 & 34 & 0.000049903 \\ 45 & 11562 & 498639 & 801 & 497838 & 35 & 0.000070196 \\ 46 & 16735 & 366561 & 150 & 366411 & 29 & 0.000079120 \\ 47 & 34245 & 478242 & 0 & 478242 & 42 & 0.000087829 \\ 48 & 17408 & 292548 & 12 & 292536 & 27 & 0.000092301 \\ 49 & 8359 & 8308 & 6 & 8302 & 1 & 0.00012038 \\ 50 & 17888 & 20982 & 1 & 20981 & 3 & 0.000143 \\ 51 & 17816 & 13487 & 5 & 13482 & 2 & 0.000148313 \\ 52 & 9989 & 102585 & 4 & 102581 & 19 & 0.000185247 \\ 53 & 24493 & 10257 & 1 & 10256 & 2 & 0.000195027 \\ 54 & 9929 & 45064 & 975 & 44089 & 9 & 0.000199756 \\ 55 & 577 & 143152 & 55 & 143097 & 33 & 0.000230577 \\ 56 & 5459 & 253373 & 138 & 253235 & 59 & 0.000232913 \\ 57 & 3561 & 129802 & 48 & 129754 & 32 & 0.00024659 \\ 58 & 35254 & 22288 & 2 & 22286 & 6 & 0.000269276 \\ 59 & 9325 & 577140 & 196 & 576944 & 158 & 0.000273839 \\ 60 & 15412 & 547851 & 768 & 547083 & 152 & 0.000277525 \\ 61 & 286 & 222119 & 1487 & 220632 & 63 & 0.000283712 \\ 62 & 3491 & 129975 & 240 & 129735 & 38 & 0.000292449 \\ 63 & 8235 & 39533 & 0 & 39533 & 13 & 0.000328947 \\ 64 & 29686 & 241832 & 18 & 241814 & 82 & 0.000339193 \\ 65 & 38930 & 2871 & 9 & 2862 & 1 & 0.000348432 \\ 66 & 20959 & 653915 & 35814 & 618101 & 240 & 0.000367155 \\ 67 & 16086 & 10547 & 0 & 10547 & 4 & 0.000379399 \\ 68 & 9304 & 10256 & 1 & 10255 & 4 & 0.000390168 \\ 69 & 5391 & 416184 & 1157 & 415027 & 164 & 0.000394212 \\ 70 & 8732 & 14512 & 2 & 14510 & 6 & 0.000413622 \\ 71 & 6458 & 95430 & 1083 & 94347 & 42 & 0.000440307 \\ 72 & 6461 & 338183 & 449 & 337734 & 155 & 0.000458542 \\ 73 & 34695 & 721036 & 473 & 720563 & 331 & 0.000459273\end{array}$




\begin{tabular}{|c|c|c|c|c|c|}
\hline 2914 & 413011 & 336 & 412675 & 192 & 0.000465095 \\
\hline 12989 & 187108 & 88 & 187020 & 89 & 0.000475887 \\
\hline 16150 & 110817 & 15 & 110802 & 54 & 0.000487527 \\
\hline 9505 & 154955 & 8 & 154947 & 78 & 0.000503625 \\
\hline 41865 & 382586 & 50 & 382536 & 201 & 0.000525648 \\
\hline 44957 & 193280 & 510 & 192770 & 110 & 0.000569447 \\
\hline 4628 & 15179 & 2 & 15177 & 9 & 0.000593276 \\
\hline 4651 & 98542 & 10 & 98532 & 59 & 0.000599088 \\
\hline 20485 & 22062 & 64 & 21998 & 14 & 0.000634978 \\
\hline 9002 & 44302 & 216 & 44086 & 30 & 0.000677629 \\
\hline 6762 & 1731856 & 5829 & 1726027 & 1226 & 0.000708413 \\
\hline 20569 & 755804 & 112 & 755692 & 599 & 0.000793162 \\
\hline 7474 & 5044 & 0 & 5044 & 4 & 0.000793651 \\
\hline 4847 & 104530 & 692 & 103838 & 84 & 0.000804243 \\
\hline 12252 & 649183 & 4350 & 644833 & 552 & 0.000851023 \\
\hline 3257 & 791261 & 1384 & 789877 & 722 & 0.000913301 \\
\hline 3292 & 1048892 & 106372 & 942520 & 959 & 0.000915135 \\
\hline 7473 & 1883764 & 2902 & 1880862 & 1749 & 0.000929323 \\
\hline 8404 & 757908 & 273 & 757635 & 708 & 0.000935024 \\
\hline 12956 & 1209412 & 1325 & 1208087 & 1133 & 0.000937697 \\
\hline 9121 & 491253 & 15642 & 475611 & 474 & 0.000965811 \\
\hline 4808 & 276856 & 167 & 276689 & 292 & 0.001055813 \\
\hline 6453 & 827269 & 3436 & 823833 & 887 & 0.001073353 \\
\hline 5617 & 742527 & 96094 & 646433 & 813 & 0.00109611 \\
\hline 1299 & 2214237 & 2143 & 2212094 & 2512 & 0.001135765 \\
\hline 3549 & 1868710 & 1803 & 1866907 & 2128 & 0.001140052 \\
\hline 8961 & 95505 & 1 & 95504 & 109 & 0.001142606 \\
\hline 6830 & 786280 & 67 & 786213 & 943 & 0.001200758 \\
\hline 5511 & 908286 & 5265 & 903021 & 1097 & 0.00120923 \\
\hline 3356 & 1397337 & 52562 & 1344775 & 1688 & 0.001209473 \\
\hline 6746 & 280616 & 270 & 280346 & 341 & 0.001216662 \\
\hline 4637 & 1137421 & 1918 & 1135503 & 1398 & 0.001230609 \\
\hline 3320 & 1951850 & 2493 & 1949357 & 2459 & 0.00126142 \\
\hline 20612 & 7094 & 1 & 7093 & 9 & 0.001270289 \\
\hline 17431 & 191397 & 773 & 190624 & 244 & 0.001276464 \\
\hline 13046 & 519128 & 1636 & 517492 & 672 & 0.001296156 \\
\hline 9831 & 654842 & 819 & 654023 & 855 & 0.001307365 \\
\hline 3303 & 2332719 & 24140 & 2308579 & 3209 & 0.001377543 \\
\hline 27699 & 61755 & 6 & 61749 & 86 & 0.001394542 \\
\hline 7018 & 658316 & 577 & 657739 & 957 & 0.001455826 \\
\hline 3758 & 1226111 & 1173 & 1224938 & 1860 & 0.001519296 \\
\hline 115 & 2286085 & 98397 & 2187688 & 3607 & 0.0015803 \\
\hline 5603 & 659535 & 756 & 658779 & 1043 & 0.001583922 \\
\hline 5588 & 98559 & 43 & 98516 & 163 & 0.001656571 \\
\hline 1239 & 742906 & 2520 & 740386 & 1274 & 0.001717833 \\
\hline 4648 & 303148 & 22 & 303126 & 539 & 0.001781176 \\
\hline 2119 & 100403 & 3523 & 96880 & 180 & 0.001795995 \\
\hline 8220 & 138828 & 84221 & 54607 & 249 & 0.001796809 \\
\hline 17964 & 100108 & 4714 & 95394 & 180 & 0.001801297 \\
\hline 8685 & 289314 & 17143 & 272171 & 524 & 0.001814467 \\
\hline
\end{tabular}




\begin{tabular}{|c|c|c|c|c|c|}
\hline 34209 & 3304 & 45 & 3259 & 6 & 0.001819284 \\
\hline 42979 & 72181 & 490 & 71691 & 135 & 0.001873803 \\
\hline 5416 & 100443 & 362 & 100081 & 189 & 0.001885212 \\
\hline 14080 & 200615 & 514 & 200101 & 384 & 0.001917785 \\
\hline 34726 & 97823 & 12 & 97811 & 193 & 0.001976851 \\
\hline 3216 & 646471 & 4668 & 641803 & 1280 & 0.001983909 \\
\hline 7568 & 93121 & 11 & 93110 & 197 & 0.002120012 \\
\hline 18101 & 99256 & 60 & 99196 & 221 & 0.002231534 \\
\hline 3790 & 100290 & 2114 & 98176 & 226 & 0.002258555 \\
\hline 23216 & 535177 & 4504 & 530673 & 1217 & 0.002279197 \\
\hline 8400 & 140352 & 2771 & 137581 & 334 & 0.002385408 \\
\hline 1120 & 7124 & 0 & 7124 & 17 & 0.002392008 \\
\hline 8928 & 543933 & 410 & 543523 & 1316 & 0.002425283 \\
\hline 12389 & 96712 & 223 & 96489 & 234 & 0.002425423 \\
\hline 4837 & 687470 & 2519 & 684951 & 1676 & 0.002443883 \\
\hline 15857 & 609421 & 175 & 609246 & 1511 & 0.002485565 \\
\hline 2905 & 602543 & 1456 & 601087 & 1499 & 0.002493994 \\
\hline 5483 & 758951 & 283 & 758668 & 1921 & 0.002537548 \\
\hline 8422 & 99264 & 220 & 99044 & 260 & 0.002626157 \\
\hline 12880 & 98234 & 51 & 98183 & 271 & 0.002766351 \\
\hline 8151 & 126060 & 8 & 126052 & 354 & 0.002816095 \\
\hline 6667 & 1746 & 8 & 1738 & 5 & 0.002871913 \\
\hline 12741 & 624069 & 409 & 623660 & 1818 & 0.002921651 \\
\hline 7643 & 396905 & 19342 & 377563 & 1159 & 0.002928646 \\
\hline 3786 & 575213 & 6998 & 568215 & 1810 & 0.003156593 \\
\hline 174 & 1017581 & 2909 & 1014672 & 3325 & 0.003278265 \\
\hline 4788 & 746018 & 6562 & 739456 & 2607 & 0.003506808 \\
\hline 4589 & 296499 & 2811 & 293688 & 1037 & 0.003509758 \\
\hline 9911 & 659768 & 287 & 659481 & 2328 & 0.003541008 \\
\hline 27737 & 99029 & 6 & 99023 & 351 & 0.003557024 \\
\hline 1257 & 95470 & 10 & 95460 & 342 & 0.003595156 \\
\hline 4657 & 6034 & 1 & 6033 & 22 & 0.003659348 \\
\hline 8778 & 757184 & 254 & 756930 & 2769 & 0.003670394 \\
\hline 4134 & 404550 & 238 & 404312 & 1559 & 0.003868573 \\
\hline 701 & 1841843 & 786 & 1841057 & 7528 & 0.004103984 \\
\hline 2516 & 100069 & 15 & 100054 & 417 & 0.004184562 \\
\hline 31549 & 99902 & 1821 & 98081 & 419 & 0.004211775 \\
\hline 6713 & 99356 & 23 & 99333 & 435 & 0.004397448 \\
\hline 11664 & 100423 & 2580 & 97843 & 456 & 0.004561505 \\
\hline 3327 & 25911 & 155 & 25756 & 131 & 0.005081458 \\
\hline 10212 & 200048 & 240 & 199808 & 1054 & 0.005296642 \\
\hline 15623 & 200207 & 99461 & 100746 & 1069 & 0.005368137 \\
\hline 12558 & 98438 & 4997 & 93441 & 530 & 0.005413245 \\
\hline 16265 & 5762 & 0 & 5762 & 33 & 0.005760168 \\
\hline 20002 & 100045 & 4915 & 95130 & 584 & 0.005871648 \\
\hline 6774 & 105021 & 8 & 105013 & 661 & 0.006333844 \\
\hline 6429 & 299745 & 5292 & 294453 & 1908 & 0.006406189 \\
\hline 18566 & 308 & 0 & 308 & 2 & 0.006535948 \\
\hline 8452 & 513613 & 4318 & 509295 & 3453 & 0.006768465 \\
\hline 5610 & 853301 & 5039 & 848262 & 5753 & 0.006787816 \\
\hline
\end{tabular}




\begin{tabular}{|c|c|c|c|c|c|c|}
\hline 174 & 1273 & 136708 & 119 & 136589 & 971 & 0.00715354 \\
\hline 175 & 9092 & 100172 & 429 & 99743 & 752 & 0.00756387 \\
\hline 176 & 24739 & 99843 & 26 & 99817 & 775 & 0.00782291 \\
\hline 177 & 9931 & 98518 & 119 & 98399 & 886 & 0.009074893 \\
\hline 178 & 4812 & 292637 & 178575 & 114062 & 2797 & 0.009650152 \\
\hline 179 & 25086 & 99405 & 24087 & 75318 & 953 & 0.009679844 \\
\hline 180 & 8437 & 100445 & 17 & 100428 & 986 & 0.009913633 \\
\hline 181 & 3269 & 436050 & 108407 & 327643 & 4289 & 0.009933736 \\
\hline 182 & 1221 & 423227 & 438 & 422789 & 4293 & 0.010247438 \\
\hline 183 & 17726 & 100256 & 1356 & 98900 & 1020 & 0.010278528 \\
\hline 184 & 5400 & 170142 & 1781 & 168361 & 1766 & 0.010488431 \\
\hline 185 & 6057 & 100301 & 5277 & 95024 & 1072 & 0.010803293 \\
\hline 186 & 21724 & 200581 & 20 & 200561 & 2155 & 0.010860472 \\
\hline 187 & 8483 & 948262 & 1747 & 946515 & 10492 & 0.011188244 \\
\hline 188 & 7303 & 99460 & 5 & 99455 & 1133 & 0.011522776 \\
\hline 189 & 5602 & 99879 & 25 & 99854 & 1167 & 0.011822271 \\
\hline 190 & 9186 & 100429 & 14 & 100415 & 1219 & 0.012287068 \\
\hline 191 & 8514 & 100455 & 9 & 100446 & 1221 & 0.012304251 \\
\hline 192 & 2820 & 100365 & 17257 & 83108 & 1329 & 0.013419363 \\
\hline 193 & 3215 & 100451 & 46 & 100405 & 1331 & 0.013428168 \\
\hline 194 & 8866 & 99059 & 1357 & 97702 & 1323 & 0.013536466 \\
\hline 195 & 23889 & 100435 & 225 & 100210 & 1358 & 0.013706511 \\
\hline 196 & 8386 & 194749 & 104264 & 90485 & 2699 & 0.014053632 \\
\hline 197 & 10026 & 220894 & 17 & 220877 & 3306 & 0.015193853 \\
\hline 198 & 6568 & 100414 & 277 & 100137 & 1603 & 0.01622289 \\
\hline 199 & 4732 & 100384 & 1774 & 98610 & 1633 & 0.016536541 \\
\hline 200 & 5388 & 100393 & 33 & 100360 & 1775 & 0.017998743 \\
\hline 201 & 2588 & 99817 & 1925 & 97892 & 1790 & 0.018260275 \\
\hline 202 & 12530 & 100401 & 31 & 100370 & 1833 & 0.018596299 \\
\hline 203 & 4766 & 676544 & 318 & 676226 & 12623 & 0.019012804 \\
\hline 204 & 36879 & 95634 & 2179 & 93455 & 1792 & 0.019095927 \\
\hline 205 & 9830 & 100341 & 4 & 100337 & 2129 & 0.021677595 \\
\hline 206 & 11486 & 100459 & 6 & 100453 & 2141 & 0.021776277 \\
\hline 207 & 12310 & 400094 & 16016 & 384078 & 9130 & 0.023352534 \\
\hline 208 & 4750 & 100423 & 33 & 100390 & 2381 & 0.02428551 \\
\hline 209 & 5606 & 100372 & 119 & 100253 & 2395 & 0.024444512 \\
\hline 210 & 16206 & 100421 & 5 & 100416 & 2424 & 0.024735451 \\
\hline 211 & 33861 & 100221 & 4 & 100217 & 2432 & 0.024869873 \\
\hline 212 & 5432 & 301399 & 47 & 301352 & 7696 & 0.026203341 \\
\hline 213 & 3300 & 97592 & 1020 & 96572 & 2527 & 0.026581812 \\
\hline 214 & 2609 & 100432 & 867 & 99565 & 2767 & 0.028331541 \\
\hline 215 & 10429 & 100427 & 3565 & 96862 & 2805 & 0.028733277 \\
\hline 216 & 8447 & 100405 & 35 & 100370 & 2813 & 0.028824084 \\
\hline 217 & 8289 & 100435 & 22 & 100413 & 3218 & 0.033101207 \\
\hline 218 & 14551 & 100307 & 123 & 100184 & 3538 & 0.036561295 \\
\hline 219 & 1836 & 100422 & 28 & 100394 & 4868 & 0.050945015 \\
\hline 220 & 5384 & 100412 & 75 & 100337 & 4997 & 0.05237122 \\
\hline 221 & 19262 & 100447 & 31 & 100416 & 5368 & 0.056458314 \\
\hline 222 & 2828 & 21396 & 21396 & 0 & 0 & 0 \\
\hline 223 & 12491 & 5 & 5 & 0 & 0 & 0 \\
\hline
\end{tabular}




\section{D.2 Inter-AS Quality}

This table contains the complete ranking of the inter-AS qualities as described in Sect. 4.6.2

$\begin{array}{llll}\text { Rank } & \text { ASN } & \text { routing count } & \text { inter-AS quality } \\ 1 & 8501 & 3264 & 0 \\ 2 & 13284 & 8 & 0 \\ 3 & 41398 & 3264 & 0 \\ 4 & 3786 & 314902 & 0.000088441 \\ 5 & 9831 & 361377 & 0.000217192 \\ 6 & 513 & 3541 & 0.000283712 \\ 7 & 6667 & 1475 & 0.000439267 \\ 8 & 15857 & 217284 & 0.000525648 \\ 9 & 34209 & 3215 & 0.000527884 \\ 10 & 13046 & 305660 & 0.00056591 \\ 11 & 24493 & 9849 & 0.000599088 \\ 12 & 12555 & 453 & 0.000634978 \\ 13 & 17408 & 193440 & 0.000695801 \\ 14 & 4837 & 173390 & 0.000919683 \\ 15 & 23216 & 280781 & 0.000937699 \\ 16 & 9911 & 397378 & 0.00094493 \\ 17 & 3758 & 646359 & 0.000972016 \\ 18 & 5391 & 308213 & 0.000980223 \\ 19 & 3269 & 92036 & 0.000986743 \\ 20 & 8483 & 371724 & 0.001000186 \\ 21 & 17964 & 54237 & 0.001085716 \\ 22 & 16260 & 30928 & 0.001098667 \\ 23 & 20569 & 386258 & 0.001100048 \\ 24 & 12252 & 211011 & 0.001140107 \\ 25 & 5384 & 1 & 0.001142606 \\ 26 & 3292 & 382789 & 0.0011546 \\ 27 & 4808 & 195660 & 0.001157996 \\ 28 & 5617 & 62559 & 0.001180922 \\ 29 & 5610 & 377486 & 0.001185437 \\ 30 & 8404 & 189096 & 0.001200758 \\ 31 & 22773 & 4 & 0.001209473 \\ 32 & 12310 & 232502 & 0.001216662 \\ 33 & 1221 & 180259 & 0.001234541 \\ 34 & 5603 & 452987 & 0.001239161 \\ 35 & 12741 & 276607 & 0.001242733 \\ 36 & 15412 & 298223 & 0.001292496 \\ 37 & 3327 & 13917 & 0.001349093 \\ 38 & 8452 & 279414 & 0.001373776 \\ 39 & 8151 & 7744 & 0.001377543 \\ 40 & 9325 & 371760 & 0.001377543 \\ 41 & 21724 & 15180 & 0.001377543 \\ 42 & 23520 & 5932 & 0.001377543 \\ 43 & 31549 & 300 & 0.001377543 \\ 44 & 16735 & 236726 & 0.001377567 \\ & & & \end{array}$




$\begin{array}{llll}45 & 11562 & 237111 & 0.001377619 \\ 46 & 10429 & 52881 & 0.001394542 \\ 47 & 5432 & 58 & 0.001406705 \\ 48 & 41865 & 298791 & 0.001478952 \\ 49 & 24748 & 4804 & 0.001628944 \\ 50 & 8778 & 441281 & 0.001671963 \\ 51 & 4648 & 7026 & 0.001680528 \\ 52 & 7473 & 1201387 & 0.001695095 \\ 53 & 8685 & 3 & 0.001736629 \\ 54 & 4788 & 396725 & 0.001738019 \\ 55 & 10026 & 86011 & 0.001771244 \\ 56 & 8434 & 3534 & 0.001795995 \\ 57 & 9092 & 31866 & 0.001796856 \\ 58 & 4847 & 49575 & 0.001801297 \\ 59 & 17816 & 10759 & 0.001801297 \\ 60 & 7018 & 313894 & 0.001859346 \\ 61 & 4766 & 438718 & 0.001864886 \\ 62 & 2588 & 1 & 0.001873803 \\ 63 & 209 & 16692 & 0.001968689 \\ 64 & 8732 & 3027 & 0.001976851 \\ 65 & 12714 & 64 & 0.001976851 \\ 66 & 29076 & 2 & 0.001976851 \\ 67 & 20959 & 230986 & 0.001981824 \\ 68 & 9304 & 6654 & 0.001998988 \\ 69 & 2905 & 349446 & 0.002169121 \\ 70 & 9989 & 79638 & 0.002224988 \\ 71 & 3561 & 63903 & 0.00224285 \\ 72 & 27908 & 14 & 0.002258555 \\ 73 & 2914 & 205189 & 0.002322777 \\ 74 & 2607 & 3264 & 0.002425283 \\ 75 & 6731 & 4 & 0.002425423 \\ 76 & 8342 & 1773 & 0.002425423 \\ 77 & 39821 & 1294 & 0.002425423 \\ 78 & 8359 & 6378 & 0.002427116 \\ 79 & 9929 & 32315 & 0.002443883 \\ 80 & 702 & 1274408 & 0.002450751 \\ 81 & 4637 & 640999 & 0.002616036 \\ 82 & 6568 & 2 & 0.002682702 \\ 83 & 6830 & 416517 & 0.003015073 \\ 84 & 3320 & 944246 & 0.003136254 \\ 85 & 34245 & 314867 & 0.003156593 \\ 86 & 5459 & 196227 & 0.003213601 \\ 87 & 3491 & 87230 & 0.00327698 \\ 88 & 4589 & 2 & 0.003392537 \\ 89 & 6461 & 228567 & 0.003402746 \\ 90 & 7643 & 216412 & 0.00343854 \\ 91 & 9505 & 102946 & 0.003484888 \\ 92 & 4635 & 1828 & 0.003506808 \\ 93 & 12322 & 1 & 0.003509758 \\ 94 & 6458 & 11666 & 0.003557024\end{array}$




$\begin{array}{llll}95 & 3303 & 1470403 & 0.003618974 \\ 96 & 29686 & 149850 & 0.003822533 \\ 97 & 17888 & 18708 & 0.003868573 \\ 98 & 6762 & 1119798 & 0.004157702 \\ 99 & 4713 & 1 & 0.004184562 \\ 100 & 12880 & 79847 & 0.004211775 \\ 101 & 34695 & 561544 & 0.00449113 \\ 102 & 3549 & 1058712 & 0.004706734 \\ 103 & 16150 & 80041 & 0.004707872 \\ 104 & 9121 & 291983 & 0.004742192 \\ 105 & 5483 & 106573 & 0.004914568 \\ 106 & 17431 & 146463 & 0.005296613 \\ 107 & 16265 & 2870 & 0.005368137 \\ 108 & 20612 & 1649 & 0.005368137 \\ 109 & 38930 & 791 & 0.005368137 \\ 110 & 3257 & 514129 & 0.005553807 \\ 111 & 11664 & 2 & 0.005871648 \\ 112 & 19422 & 17 & 0.005871648 \\ 113 & 1239 & 360767 & 0.005952071 \\ 114 & 5511 & 296127 & 0.005957379 \\ 115 & 9002 & 24713 & 0.006370361 \\ 116 & 4134 & 122788 & 0.006494653 \\ 117 & 12989 & 132613 & 0.006526247 \\ 118 & 12956 & 816816 & 0.006662498 \\ 119 & 8928 & 404236 & 0.007123308 \\ 120 & 1299 & 1277522 & 0.007248604 \\ 121 & 20485 & 21516 & 0.007507457 \\ 122 & 6453 & 417662 & 0.007561093 \\ 123 & 8220 & 43258 & 0.00756387 \\ 124 & 577 & 44413 & 0.008100552 \\ 125 & 8400 & 109936 & 0.008326948 \\ 126 & 174 & 262757 & 0.008547931 \\ 127 & 4651 & 80733 & 0.00907479 \\ 128 & 12389 & 49396 & 0.009679844 \\ 129 & 1257 & 74085 & 0.009913633 \\ 130 & 44957 & 63428 & 0.009933736 \\ 131 & 9931 & 80734 & 0.010278528 \\ 132 & 16086 & 10530 & 0.010487481 \\ 133 & 3356 & 841617 & 0.011205977 \\ 134 & 1273 & 113682 & 0.011364968 \\ 135 & 286 & 16378 & 0.011822271 \\ 136 & 9026 & 8 & 0.011822271 \\ 137 & 7474 & 4952 & 0.012297784 \\ 138 & 701 & 737588 & 0.012967672 \\ 139 & 34726 & 59418 & 0.013419363 \\ 140 & 8235 & 17010 & 0.013493738 \\ 141 & 3216 & 4746 & 0.013929499 \\ 142 & 1120 & 3343 & 0.015152802 \\ 143 & 4628 & 7440 & 0.015193853 \\ 144 & 2516 & 82307 & 0.015961672\end{array}$




$\begin{array}{llll}145 & 35254 & 7340 & 0.018260275 \\ 146 & 42979 & 48022 & 0.018260275 \\ 147 & 18101 & 79344 & 0.021677595 \\ 148 & 6746 & 232503 & 0.023352534 \\ 149 & 4657 & 5972 & 0.024281543 \\ 150 & 7568 & 73017 & 0.02428551 \\ 151 & 5588 & 83847 & 0.024444512 \\ 152 & 6739 & 1 & 0.024735451 \\ 153 & 8422 & 74749 & 0.024869873 \\ 154 & 5400 & 60204 & 0.025146409 \\ 155 & 6774 & 88327 & 0.026203341 \\ 156 & 27699 & 52881 & 0.028733277 \\ 157 & 8961 & 74139 & 0.05237122\end{array}$




\section{Appendix E}

\section{Scatter Plots}

For the display of the quality ratings we examined different kinds of scatter plots. First we plotted the number of measurements versus the number of caused outliers (Fig. E.1). The disadvantage of this plot is that the outlier-ratio is not directly apparent.

To include the outlier-ratio into the plot we created a bubble chart (Fig. E.2. . A bubble chart is a scatter plot which includes a third variable as the size of the plotted points. We used the outlier-ratio for the size of the bubbles; the smaller the size of the point the better the quality of the AS. The disadvantage of the bubble chart is that with a lot of data points it looks confusing due to the high density of bubbles. Furthermore, if the difference between the smallest and biggest bubbles is big, then the smallest bubbles are hardly visible on the plot. The top ten AS should be displayed as green bubbles. But since they are so much smaller than the worst ten, they are not visible on the scale of this plot.

Finally we produced a scatter graph which plots the number of measurements against the outlier-ratio (Fig. E.3). On this plot the quality as well as the reliability of the statement is directly apparent and stays clearly arranged even with a lot of data points. Therefore we use the last version of the scatter plot for the visualization of our rating results. 


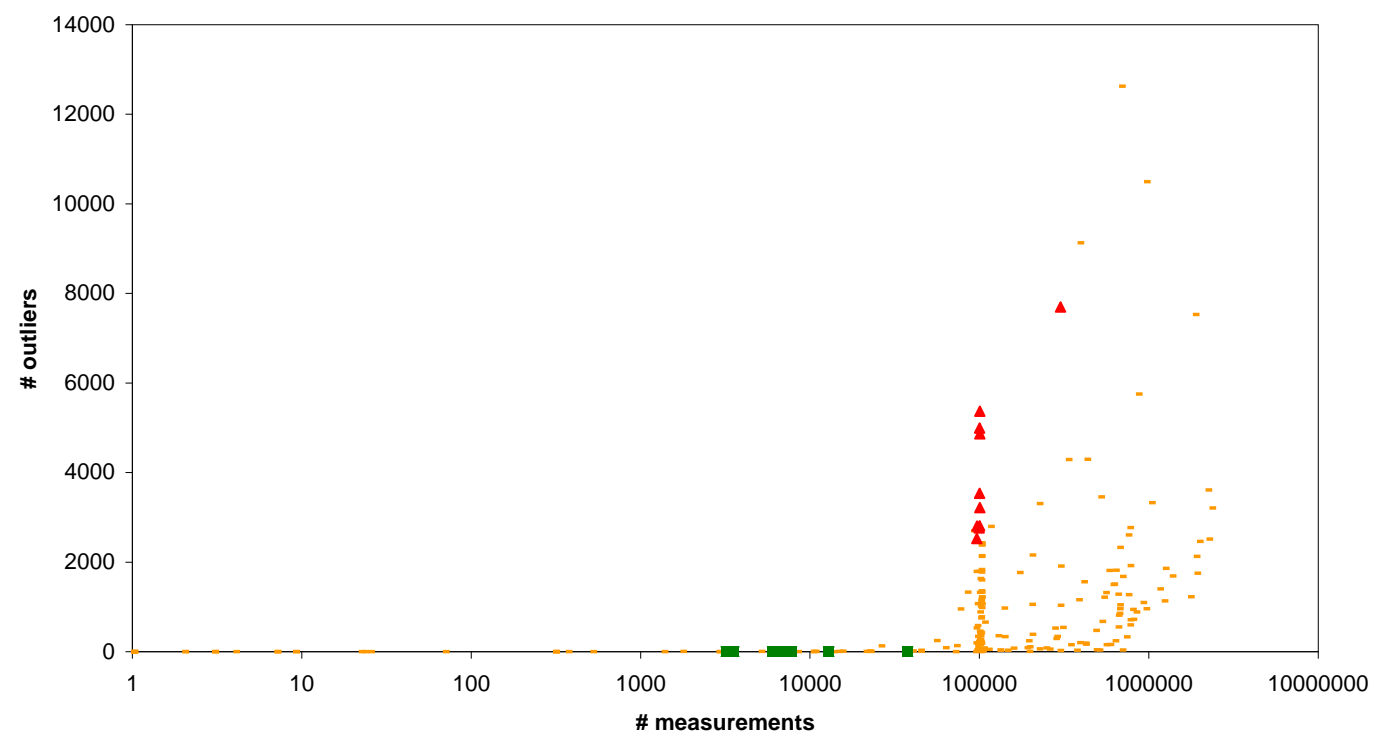

Figure E.1: Scatter plot of the intra-AS qualities with absolute values on the y-axis

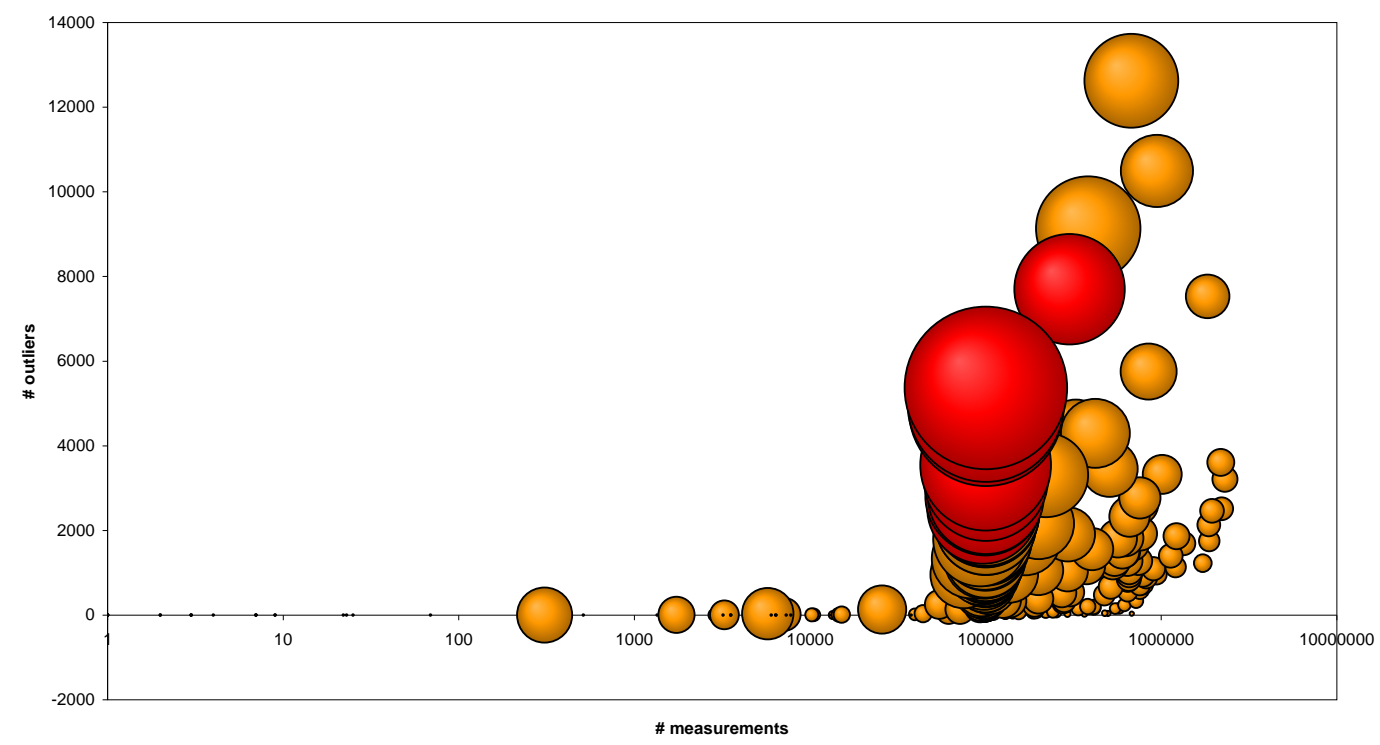

Figure E.2: Bubble chart of the intra-AS qualities 


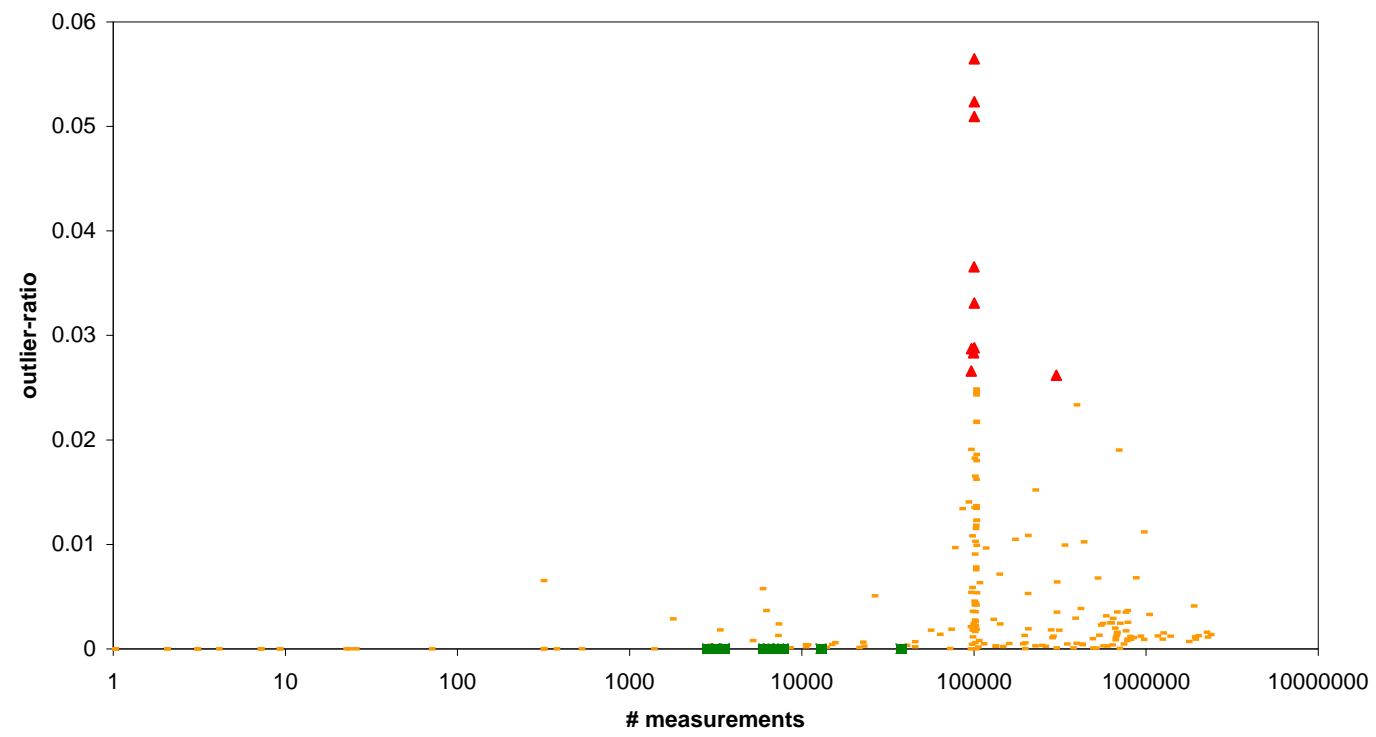

Figure E.3: Scatter plot of the intra-AS qualities with relative values on the y-axis 


\section{Appendix F}

\section{Master's Thesis Assignment}

The following pages contain the original task assignment of this master's thesis. 


\title{
Master's Thesis
}

\section{Rating Autonomous Systems}

\author{
for Laurent Zimmerli <laurent@ zimmer.li>
}

\section{Introduction}

\subsection{Bad VPN connections caused by ISP or backbone problems}

Open Systems AG based in Zurich, Switzerland is a company specialized in Internet Security since 16 years. As part of the Mission Control Services offering, Open Systems AG runs large international VPN networks for large and midsize companies, which allow different sites to securely communicate with each other. Currently Open Systems operates over 1000 devices in 80 countries.

VPN connections are tunnelled through the public Internet and therefore they are very dependent on the quality of the underlying networks and topologies of the Autonomous Systems (AS) (usually run by Internet Service Providers, ISPs). Sometimes it happens that VPN connections between customer sites become slow or fail. The customer will then complain and Open Systems has to find the reason for the poor VPN connection. The problem can have its cause on any layer of the network topology but most often the engineer finds out that the bad quality of the involved ISP links or peerings caused the problem.

\subsection{The need for rating Autonomous Systems}

Manual analysis of quality issues with VPN connections is time consuming and does not scale well with large VPN environments, consisting of hundreds of sites placed all over the world. Furthermore, it has proven difficult to choose between different ISPs for a given location because ISPs tend not to provide any quality guarantees for standard Internet connections.

Open Systems has developed several tools to measure network connection quality and ease network debugging when problems appear ([1], [2]). But ISP debugging still remains a time consuming task and extensive manual analysis is needed to get a long-term benchmark for Autonomous Systems.

The goal of this project is to automatically collect quality measurements (including historical data) and benchmark Autonomous Systems based on these measurements. This helps Open Systems (1) selecting the best ISPs to be used for any given VPN location and (2) debugging ongoing connectivity problems.

The Internet route followed by the VPN traffic between any two sites usually traverses multiple ASs. Previous work ([1]) has shown, that route changes (at the router level) do not correlate with quality changes, but that AS peering changes probably do. Therefore, this project should focus on measuring the quality of AS level routes/peerings and rating ASs using this information.

The measurement environment developed during this project should integrate with the existing Open Systems monitoring environment, which is based on Unix, Perl, Java and an SQL database. This way, historic routing data can be collected from thousands of VPN links, which should provide enough data for statistically sound conclusions. 


\subsection{Study of existing network measurement tools}

The student should acquaint himself with the previously developed measurement tools of Open Systems (e.g. [1], [2]). Laurent needs to understand the worldwide environment Open Systems is dealing with and after this study he should be able to propose a design of an automatic network measurement mechanism based on the above mentioned tools.

\subsection{Analysis of data mining methods to consolidate and store measurement data}

The main focus of Laurent's thesis lies in the collection and consolidation of the huge quantity of measurement data. Existing data mining methods that allow correlation/summarisation of measurement data of network topologies have to be analysed, tested and compared (e.g. [3]). The most promising approach can be used as inspiration for implementing the AS rating system.

\subsection{Specification of an AS rating framework}

Based on the analysis of known data mining mechanisms Laurent needs to propose a new and improved method to gather and consolidate network measurement data in a way which enables analysis of AS qualities. He needs to write a specification, such that the proposed algorithm can be used as a self-contained and automatic system that allows to rate and visualise the quality of AS. Promising algorithms and ideas that have been encountered in the analysis phase can of course be incorporated.

\subsection{Implementation of a prototype}

Following the above mentioned specification a prototype should be implemented on Linux (and run possibly also on Solaris). The resource consumption and performance of the prototype must be such that the server can still offer its normal services.

\subsection{Test of and evaluation with the prototype}

The prototype must be thoroughly tested under real network load. Therefore a small test network simulating at least three sites has to be set up.

A first objective of the testing phase is to provide a proof of concept, i.e. show that the algorithm is correct and that the whole measurement system is usable. Besides, and more importantly, the prototype provides the foundation for evaluating the whole thesis. Laurent therefore needs to define evaluation criteria and a methodology how these criteria can be verified with the prototype.

The results of the evaluation will possibly, and most probably, trigger a refinement of certain concepts, and improve the implementation. The evaluation will definitely allow issuing recommendations for future work on that topic, and what are steps to consider for an implementation beyond a prototype.

\section{Deliverables}

The following results are expected:

1. Survey of previously developed network measurement tools. Already available tools for network measurement should be studied and classified how and wether they can be used to gather quality measurements for an automatic AS rating system.

2. Survey of existing data mining concepts for network measurements. A short but precise survey should be written that studies and analyses the different known mechanisms to inventory and summarize network measurements.

3. Survey of potential AS quality issues. Possible problems that are expected or known to have an impact on AS quality. Namely, the key factors which make up a good AS. 
4. Definition of own approach to the problem. In this creative phase the student should find and document a distributed network measurement framework that allows to rate ASs.

5. Implementation of a prototype The specified prototype should be implemented.

6. Testing of the prototype Tests of the prototype with simulated network problems should be made in order to validate the functionality. The efficiency of the prototype has to be measured. Additionally, the prototype should be tested in an actual VPN environment.

7. Documentation A concise description of the work conducted in this thesis (task, related work, environment, code functionality, results and outlook). The survey as well as the description of the prototype and the testing results is part of this main documentation. The abstract of the documenttation has to be written in both English and German. The original task description is to be put in the appendix of the documentation. One sample of the documentation needs to be delivered at TIK. The whole documentation, as well as the source code, slides of the talk etc., needs to be archived in a printable, respectively executable version on a CDROM, which is to be attached to the printed documentation.

Further optional components are:

- Paper that summarizes in ten pages the task and results of this thesis.

\subsection{Documentation and presentation}

A documentation that states the steps conducted, lessons learnt, major results and an outlook on future work and unsolved problems has to be written. The code should be documented well enough such that it can be extended by another developer within reasonable time. At the end of the thesis, a presentation will have to be given at TIK that states the core tasks and results of this thesis. If important new research results are found, a paper might be written as an extract of the thesis and submitted to a computer network and security conference.

The developed code of the prototype and the implemented algorithms will be released under the terms of GPL2 as open source at the end of the thesis.

\subsection{Dates}

This Master's thesis starts on March $3^{\text {rd }} 2008$ and is finished on August $29^{\text {th }} 2008$. It lasts 26 weeks in total.

At the end of the second week Laurent has to provide a schedule for the thesis. It will be discussed with the supervisors.

After a month Laurent should provide a draft of the table of contents (ToC) of the thesis. The ToC suggests that the documentation is written in parallel to the progress of the work.

Two intermediate informal presentations for Prof. Plattner and all supervisors will be scheduled 2 months and 4 months into this thesis.

A final presentation at TIK will be scheduled close to the completion date of the thesis. The presentation consists of a 20 minutes talk and reserves 5 minutes for questions.

Informal meetings with the supervisors will be announced and organized on demand. 


\section{Supervisors}

Roel Vandewall,rv@open.ch, +41 4445574 00, Open Systems AG, http://www.open.ch Christoph Göldi, chg@open.ch, +41 4445574 00, Open Systems AG, http://www.open.ch Stefan Lampart, stl@open.ch, +41 4445574 00, Open Systems AG, http://www.open.ch Bernhard Tellenbach, betellen@tik.ee.ethz.ch, +41 4463270 06, ETZ G 97, ETH Zurich

\section{References}

[1] Janneth Malibago. Automated Monitoring of Internet Service Provider (ISP) Topologies. Master's Thesis MA-2006-11, August 3, 2006 / Summer Term 2006

[2] Dominique Giger. Passive Measurement of Network Quality. Student Thesis MA-2006-03, 6th May 2005 / Winter Term 2005/06

[3] David G. Andersen, Nick Feamster. Challenges and Opportunities in Internet Data Mining. Parallel Data Laboratory, Carnegie Mellon University, Pittsburgh, January 2006 


\section{Appendix G}

\section{Schedule}

This appendix contains the initial schedule of this master's thesis.

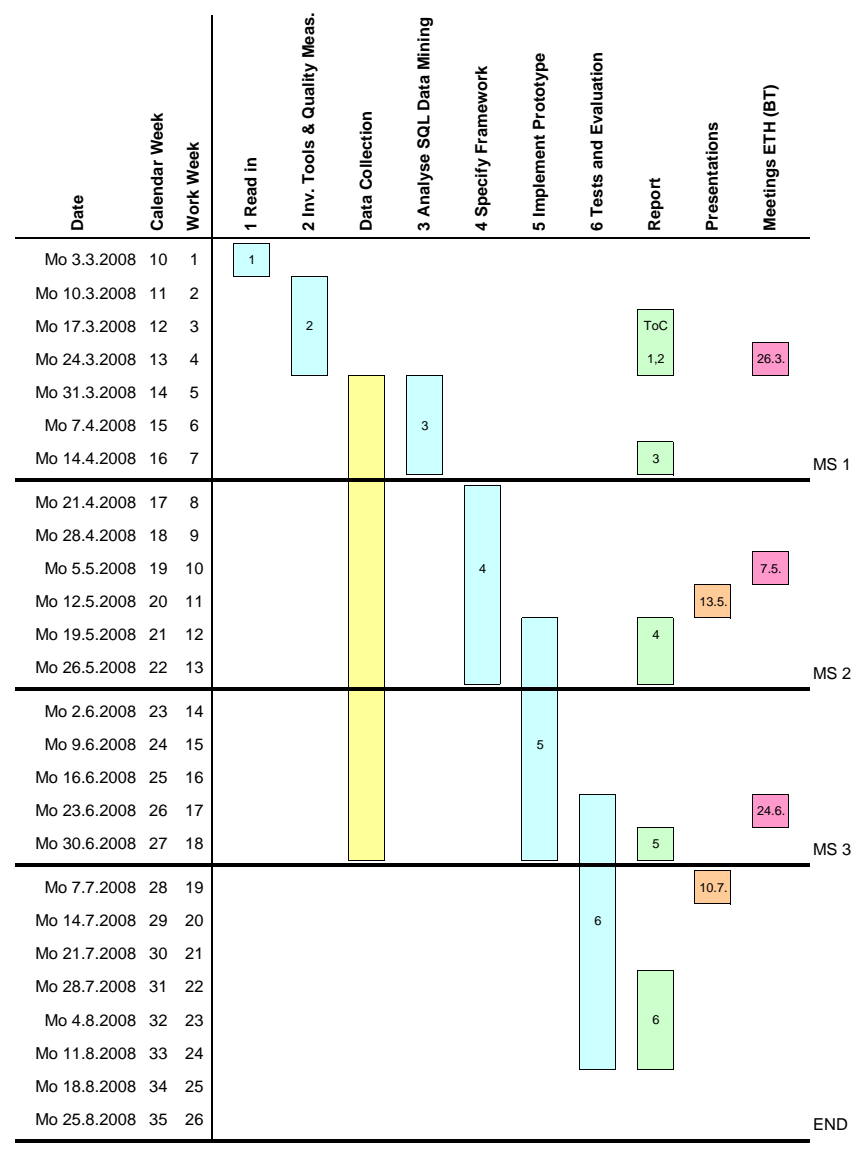

MS 1: Complete overview of related work, available tools, possible measures, reasonable methods MS 2: Complete and detailed specification of the whole framework

MS 3: Complete and usable implementation of the framework and enough evaluable data 


\section{Bibliography}

[1] N. Alves Jr., M.P. de Albuquerque, M.P. de Albuquerque. Topology and Shortest Path Length Evolution of the Internet Autonomous Systems Interconnectivity. Article to PhD thesis, October 2007

[2] X. Dimitropoulos, D. Krioukov, M. Fomenkov, B. Huffaker, Y. Hyun, k. claffy, G. Riley. AS Relationships: Inference and Validation. ACM SIGCOMM Computer Communications Review (CCR), 2007

[3] X. Dimitropoulos, D. Krioukov, G. Riley, k. claffy. Classifying the Types of Autonomous Systems in the Internet. SIGCOMM Poster, 2005

[4] Yixuan Wang, Ye Wang, Maoke Chen, and Xing Li. Inter-Domain Access Volume Model: Ranking Autonomous Systems. The International Conference on Information Networking (ICOIN), January 2007

[5] T. Chen, L. Hu. Internet Performance Monitoring. Proceedings of the IEEE, 2002

[6] R. Prasad, C. Dovrolis, M. Murray, k. claffy. Bandwidth Estimation: Metrics, Measurement Techniques, and Tools. IEEE Networks, 2003

[7] K. Harfoush, A. Bestavros, J. Byers. Measuring Bottleneck Bandwidth of Targeted Path Segments. INFOCOM 2003, Twenty-Second Annual Joint Conference of the IEEE Computer and Communications Societies, March-April 2003

[8] B. Augustin, X. Cuvellier, B. Orgogozo, F. Viger, T. Friedman, M. Latapy, C. Magnien, R. Teixeira. Avoiding Traceroute Anomalies with Paris Traceroute. Internet Measurement Conference, 2006

[9] F. Baccelli, S. Machiraju, D. Veitch, J. Bolot. The Role of PASTA in Network Measurement. Conference on Applications, technologies, architectures, and protocols for computer communications, 2006

[10] RFC 2330, Framework for IP Performance Metrics. ftp://ftp.rfc-editor.org/in-notes/rfc2330.txt, last visit August 2008

[11] L. Borzemski, L. Lubczynski, Z. Nowak. Application of Data Mining of the Analysis of Internet Path Performance. Parallel, Distributed and Network-Based Processing, 2004

[12] L. Borzemski. Data Mining in the Analysis of Internet Performance as Perceived by End-Users. Systems Engineering, 2005

[13] M. Baldi, E. Baralis, F. Risso. Data Mining Techniques for Effective and Scalable Traffic Analysis. Integrated Network Management, 2005

[14] J. Malibago. Automated Monitoring of Internet Service Provider (ISP) Topologies. Master's thesis, 2006

[15] German Wikipedia: Autonomes System.http://de.wikipedia.org/wiki/Autonomes_System last visit August 2008

[16] RFC 1930, Guidelines for creation, selection, and registration of an Autonomous System (AS). ftp://ftp.rfc-editor.org/in-notes/rfc1930.txt, last visit August 2008

[17] RFC 1812, Requirements for IP Version 4 Routers. ftp://ftp.rfc-editor.org/in-notes/rfc1812.txt, last visit August 2008

[18] Telecommunication Development Bureau, International Telecommunication Union ITU-D Study Group 2. Handbook “Teletraffic Engineering”. Revised 2006 
[19] IP Performance Metrics Working Group. http://www . ietf.org/html. charters/ippm-charter.html last visit August 2008

[20] WordNet, a lexical database for the English language. http://wordnet . princeton . edu/perl/webwn?s=data+mining last visit August 2008

[21] A. Riska. Aggregate matrix-analytic techniques and their applications. PhD Thesis, December 2002

[22] M.E. Crovella, A. Bestavros. Self-Similarity in World Wide Web Traffic: Evidence and Possible Causes. IEEE/ACM Transactions on networking, vol. 5, no. 6, December 1997

[23] M.E.J. Newman. Power laws, Pareto distributions and Zipf's law. Contemporary Physics, Vol. 46, No. 5, September-October 2005

[24] English Wikipedia: Interquartile Range.http://en.wikipedia.org/wiki/Interquartile_range. last visit August 2008

[25] English Wikipedia: Quartile.http://en.wikipedia.org/wiki/Quartile last visit August 2008

[26] Box Plot: Display of Distribution.http://www.physics.csbsju.edu/stats/box2.html, last visit August 2008

[27] R.W. Wolff. Poisson Arrivals see Time Averages. Operations Research, Vol. 30, No. 2 (Mar. - Apr., 1982), pp. 223-231

[28] R. Jain, I. Chlamtac. The $\mathrm{P}^{2}$ Algorithm for Dynamic Calculation of Quantiles and Histograms Without Storing Observations. Communications of the ACM, Volume 28, Number 10, October 1985

[29] English Wikipedia: Scatterplot. http://en.wikipedia.org/wiki/Scatterplot last visit August 2008 